\title{
EMPIRICAL VALIDATION OF AN IN SILICO MODEL PREDICTING THE FLUID DYNAMICS OF AN ILIAC ARTERY ANEURYSM
}

\author{
A Thesis \\ presented to \\ the Faculty of California Polytechnic State University, \\ San Luis Obispo
}

\author{
In Partial Fulfillment \\ of the Requirements for the Degree \\ Master of Science in Biomedical Engineering
}

by

Rachel Willis

June 2016 
(C) 2016

Rachel Willis

ALL RIGHTS RESERVED 


\section{COMMITTEE MEMBERSHIP}

TITLE:

Empirical Validation of an In Silico Model Predicting the Fluid Dynamics of an lliac Artery Aneurysm

AUTHOR: $\quad$ Rachel Willis

DATE SUBMITTED: June 2016

COMMITTEE CHAIR: David S. Clague, Ph.D.

Professor of Biomedical Engineering

Graduate Education Coordinator

COMMITTEE MEMBER: Scott Hazelwood, Ph.D.

Professor of Biomedical Engineering

COMMITTEE MEMBER: Michael D. Whitt, Ph.D., MBA

Lecturer of Biomedical Engineering 


\begin{abstract}
Empirical Validation of an In Silico Model Predicting the Fluid Dynamics of an Iliac Artery Aneurysm

Rachel Willis
\end{abstract}

\begin{abstract}
lliac artery aneurysms are considered rare and difficult to detect and treat. Prompt diagnosis and timely intervention are essential, because the incidence of rupture is as high as $50 \%$ [1]. The reported mortality rate for patients who undergo surgery for ruptured iliac artery aneurysm ranges from $50 \%$ to $70 \%[1]$. This study developed an in-vitro mechanical model of an iliac artery aneurysm to verify the accuracy of computer simulation software. Both the in vitro model and the in silico model can be used for further research to develop better treatment technology. This study also looks at the different types of iliac artery aneurysms, risk factors that contribute to the development of an iliac artery aneurysms, and current treatment options.
\end{abstract}

Keywords: Aneurysms, lliac, In-silico, In-vitro, Empirical, Vortex, Hagen-Poiseuille, Shear

Stress, Newtonian, COMSOL MULTIPHYSICS ${ }^{\circledR}$, particle tracking. 


\section{ACKNOWLEDGMENTS}

The author would like to thank Daniel Greinke for his contributions as a research partner and for providing adequate background knowledge on 3D printing. The author would also like to thank Dr. David Clague for his guidance as adviser to the project. Finally, the author would like to thank Jason Delgadillo for his assistance with Solidworks. 


\section{TABLE OF CONTENTS}

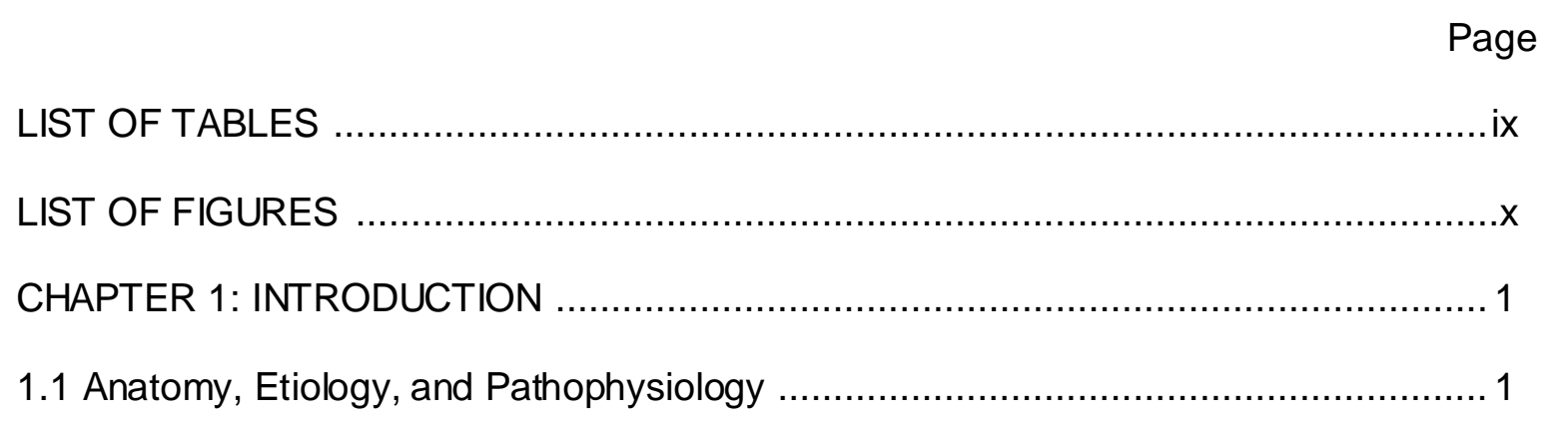

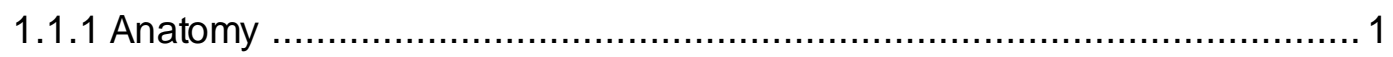

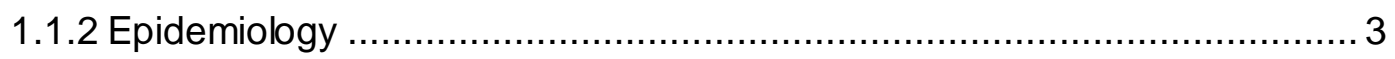

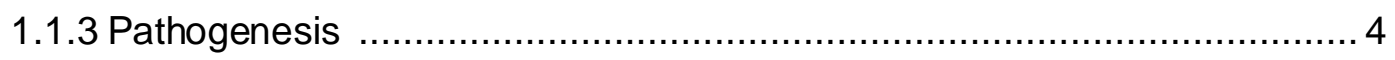

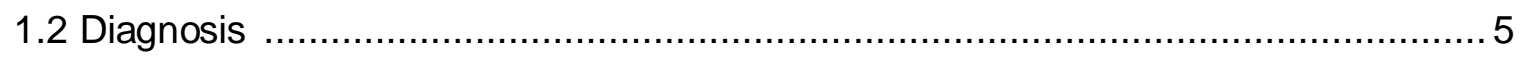

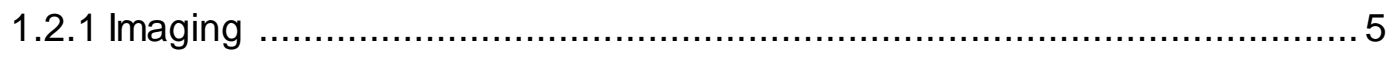

1.2.2 Classification Scheme for lliac Artery Aneurysms ............................ 6

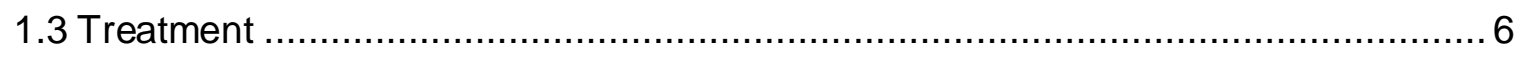

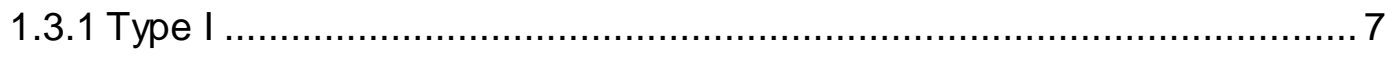

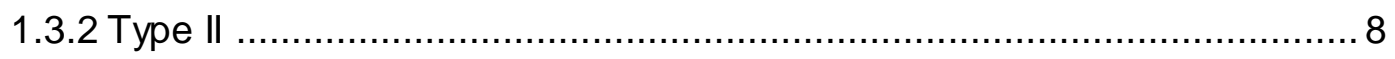

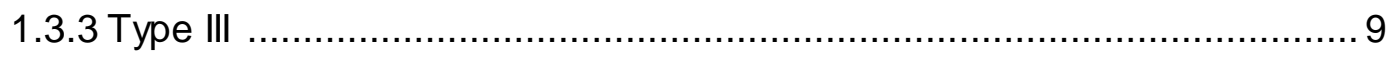

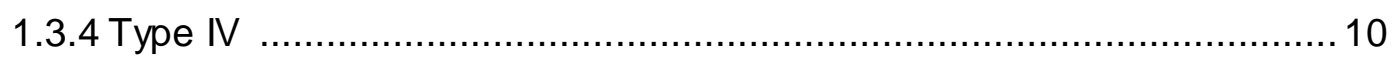

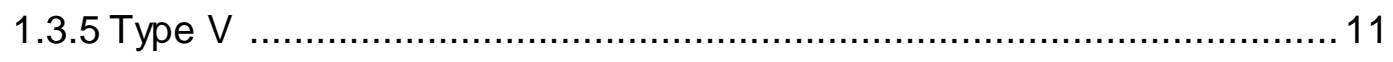

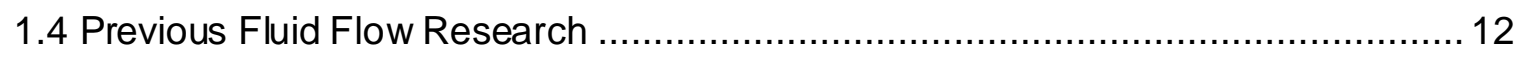

1.4.1 Mechanical Properties of lliac Aneurysms …................................. 12

1.4.2 Progression and Enlargement Rate .......................................... 13

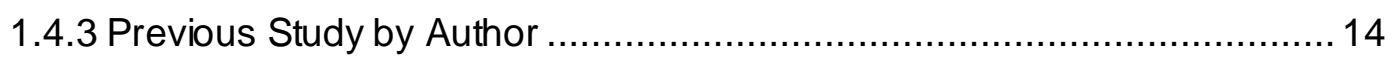

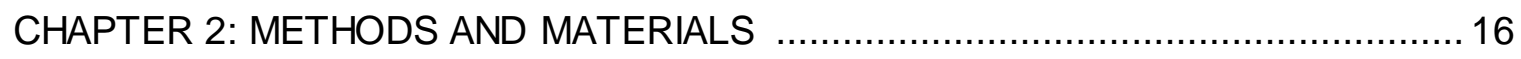

2.1 A Background on Experimental Techniques That Were Used ............................ 16

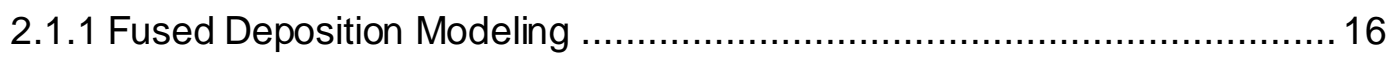

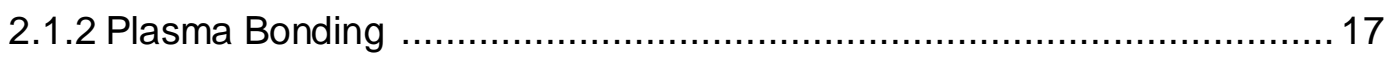




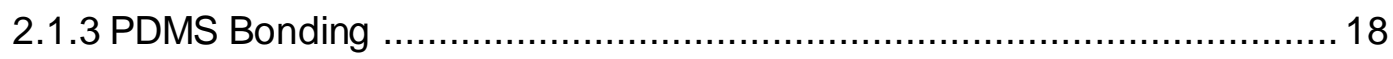

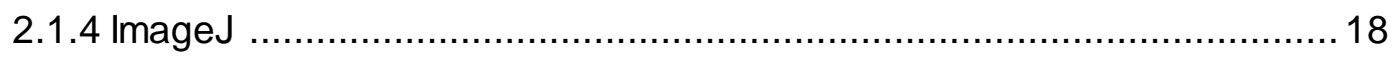

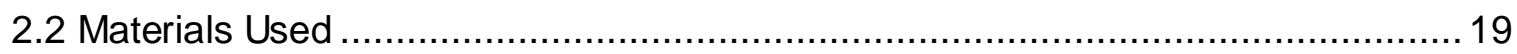

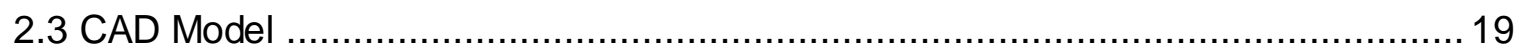

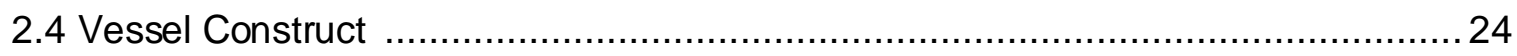

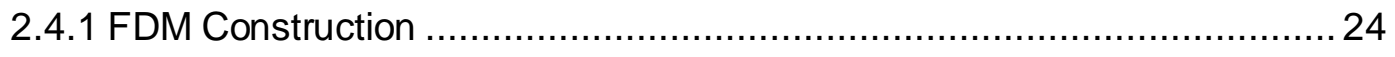

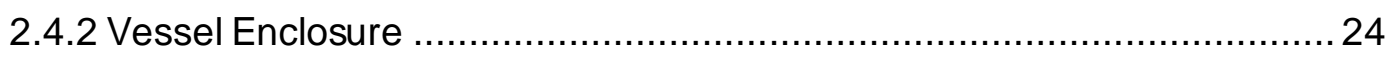

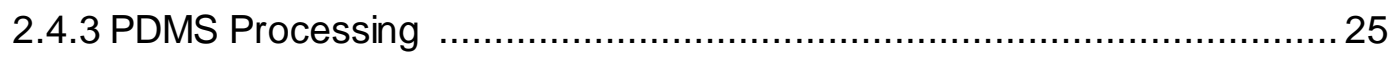

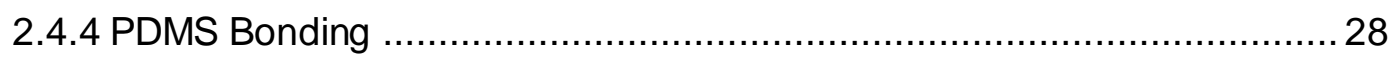

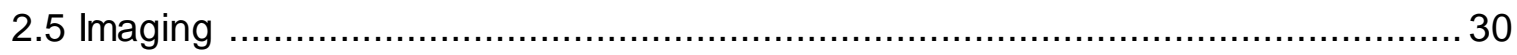

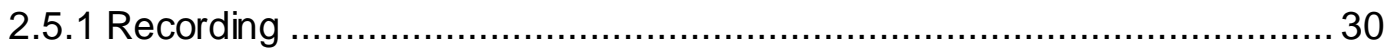

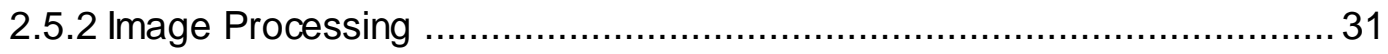

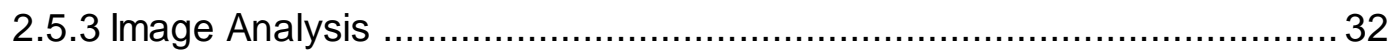

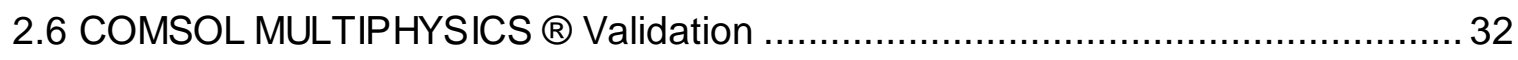

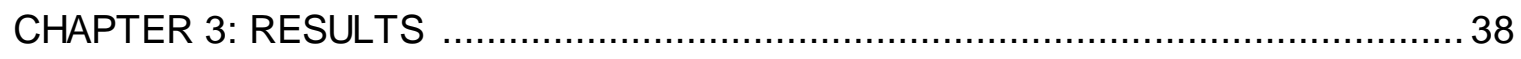

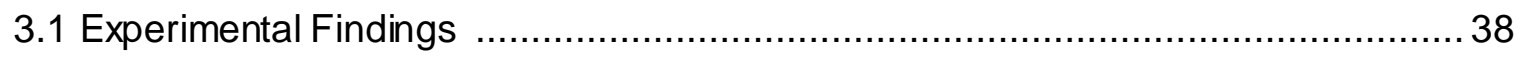

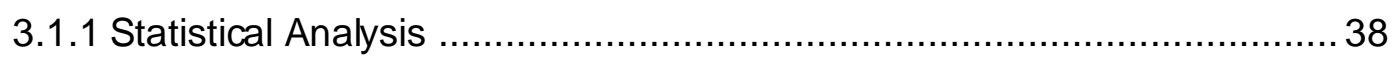

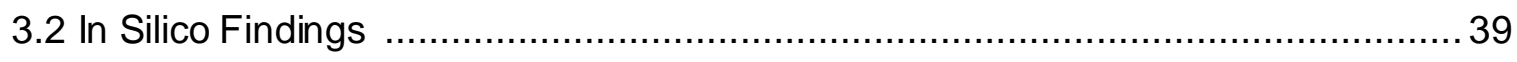

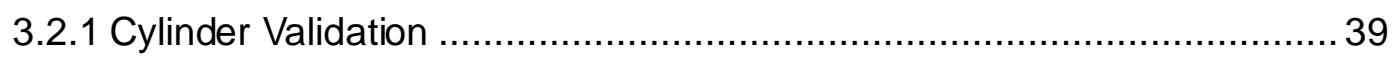

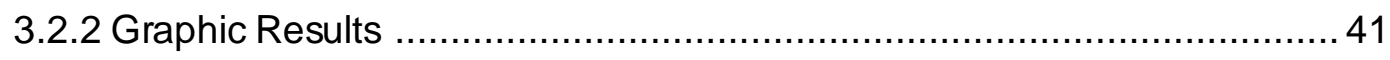

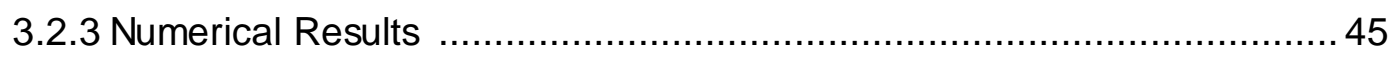

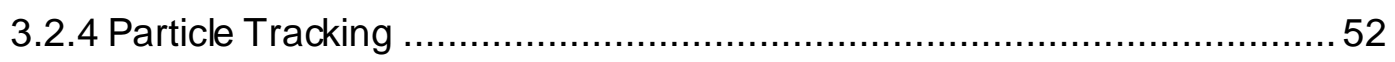

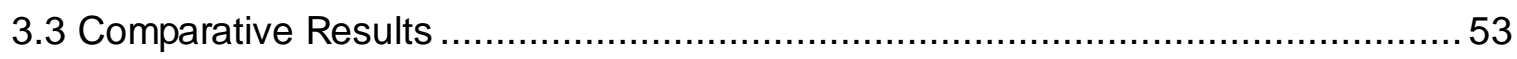

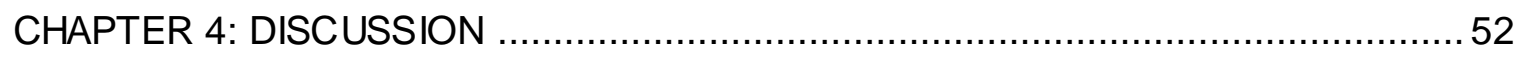

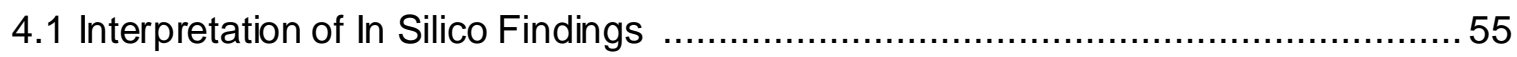

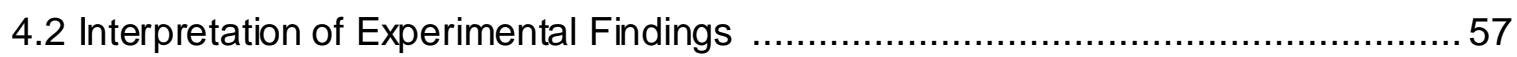




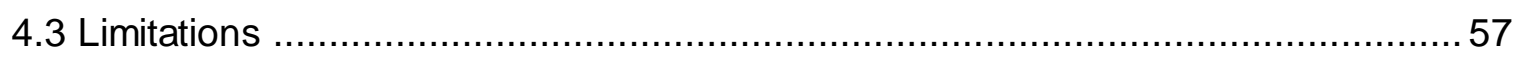

4.3.1 Limitations of the COMSOL MULTIPHYSICS $®$ Simulation ..................... 57

4.3.2 Limitations of the Mechanical Experiment ………................................ 58

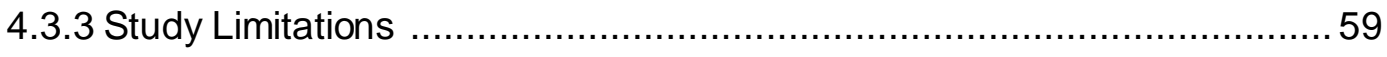

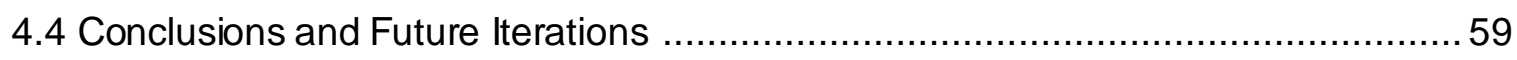

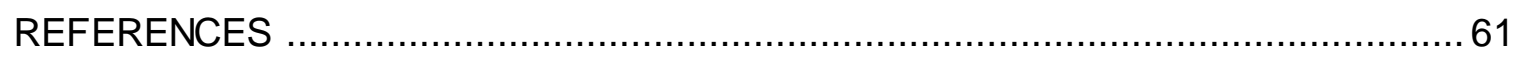
APPENDICES

A. Still Images from the Time Dependent Study ........................................... 64

B. Still Images Extracted from the Video of the Empirical Model .......................68

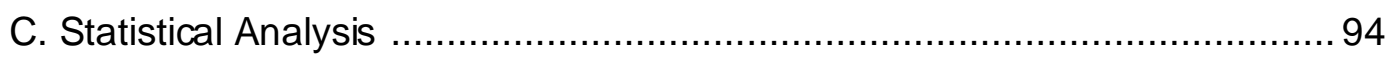

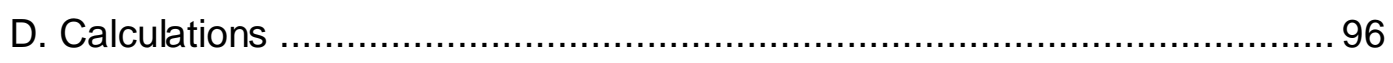

E. Image J Thresholding Processes Described in Thumbnails ......................... 97 


\section{LIST OF TABLES}

Table

Page

Table 2.1 List of Materials 19

Table 2.2 Mesh Analysis 35

Table 2.3 Number of Elements Created Based on Mesh Settings 35

Table 2.4 Flow Regime According to Reynold's Number 36

Table 3.1 Verification of COMSOL MULTIPHYSICS ® with a Cylinder Using

Common Fluid Dynamic Equations 40 


\section{LIST OF FIGURES}

Figure Page

Figure 1.1 Aneurysm Classification Based on the Form .................................... 2

Figure 1.2 Physiologic Depiction of an Artery Wall [3] ....................................... 3

Figure 1.3 How Mechanical Factors and Risk Factors Play a Role in the

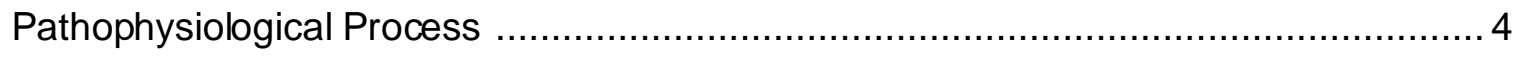

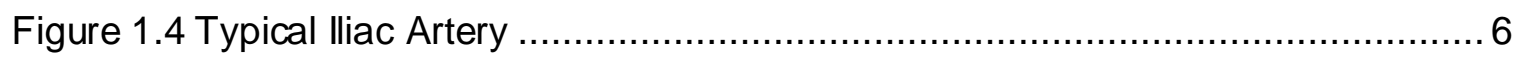

Figure 1.5 Treatment Process for a Type I IAA With a Stent Graft (left) and a

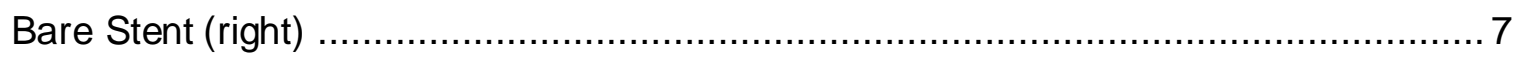

Figure 1.6 Treatment Process for a Type II IAA the Process on the Left Showing

Proximal and Distal Coiling and the Process on the Right Showing Coil Packing and

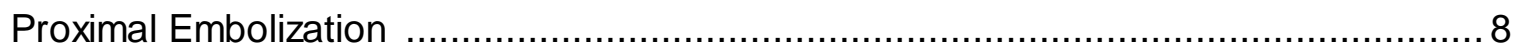

Figure 1.7 Treatment Process for a Type III IAA Stent-Graft Placement as well as

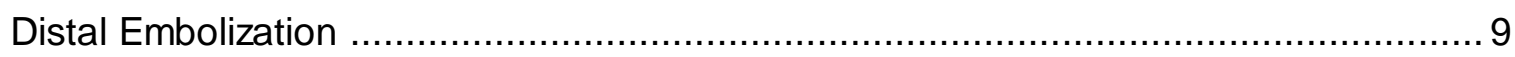

Figure 1.8 Treatment Process for a Type IV IAA Stent-Graft Placement in the Aorta

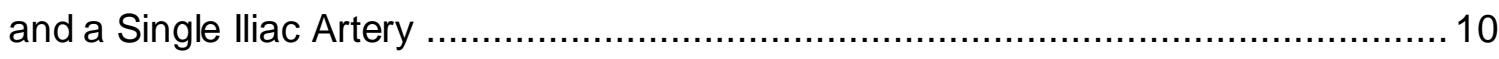

Figure 1.9 Treatment Process for a Type V IAA Common or Internal IAA that

Develops After AAA Repair with a Bifurcated Graft ........................................ 11

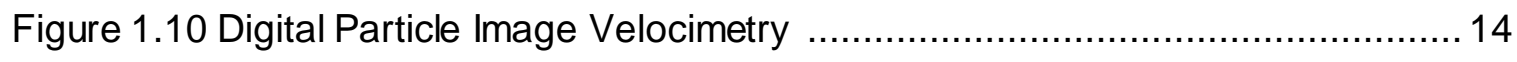

Figure 2.1 Fused Deposition Modeling Process ............................................. 16

Figure 2.2 Skeleton of the Model with Points ............................................. 20

Figure 2.3 SolidWorks Part Showing the Circles used to Create Width ...................... 21

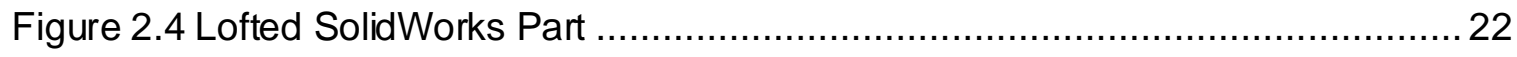

Figure 2.5 COMSOL MULTIPHYSICS $\AA$ Part Split Along the Coronal Plane ..............23

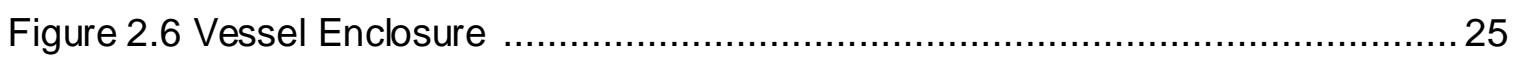

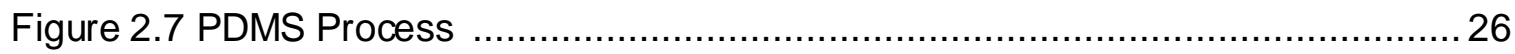


Figure 2.8 Removal of Excess PDMS Around the Printed Part 27

Figure 2.9 PDMS Molds with 3D Printed Part Removed .................................... 28

Figure 2.10 Pins Inserted into the Construct to Inhibit Drift ..................................29

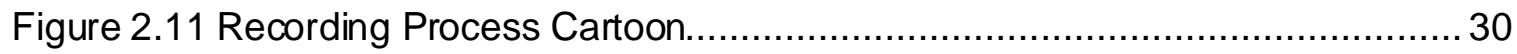

Figure 2.12 Actual Photos of the Imaging Process .............................................. 31

Figure 2.13 Fluid Properties for the Carreau Model in COMSOL MULTIPHYSICS ${ }^{\circledR}$....33

Figure 2.14 Extremely Fine Mesh in General Physics ...................................... 34

Figure 3.1 Still Image Capturing the Vortex Effect in the Aneurysm at 11 seconds ...... 38

Figure 3.2 Illustration of how the Statistics were Derived ...................................... 39

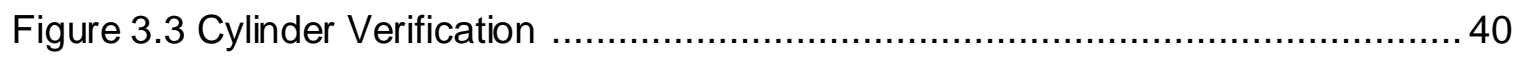

Figure 3.4 Surface Plot of Velocity in the Aneurysmal Vessel ............................... 41

Figure 3.5 Surface Plot of Velocity in the Non-aneurysmal Vessel ........................... 42

Figure 3.6 Zoomed Surface Plot of Shear Stress in Pa in the Aneurysmal Vessel ....... 42

Figure 3.7 Surface Plot of Shear Stress in Pa in the Non-aneurysmal Vessel ............. 43

Figure 3.8 Streamline Velocity of the Aneurysmal Vessel .................................. 44

Figure 3.9 Streamline Velocity in a Non-aneurysmal Vessel ................................. 44

Figure 3.10 Arrow Plot Depicting the Velocities of the Fluid .................................. 45

Figure 3.11 Blue Line Depicting the Outer Edge of the Vessel from Which the Shear

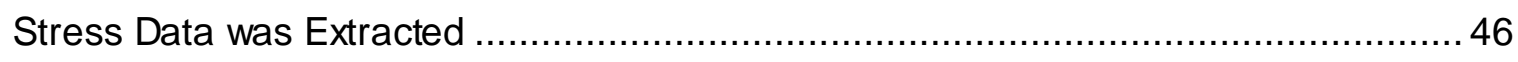

Figure 3.12 Line Graph of the Shear Stress that Occurs Along the Outer Edge

of the Vessel in the Non-aneurysmal Vessel ................................................... 46

Figure 3.13 Blue Line Depicting the Outer Edge of the Vessel from Which the Shear

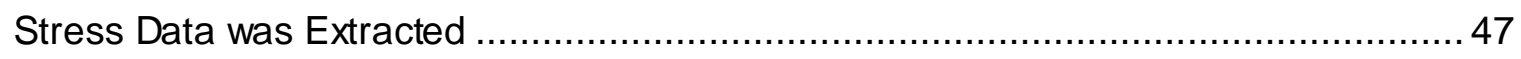

Figure 3.14 Line Graph of the Shear Stress that Occurs Along the Outer Edge

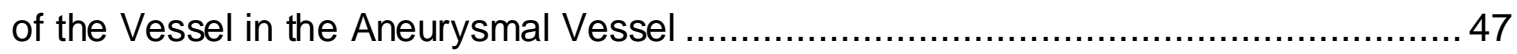

Figure 3.15 Blue Line Depicting the Inner Edge of the Vessel from Which the Shear 
Figure 3.16 Line Graph of the Shear Stress that Occurs Along the Inner Edge

of the Vessel in the Non-aneurysmal Vessel

Figure 3.17 Blue Line Depicting the Inner Edge of the Vessel from Which the Shear

Stress Data was Extracted

Figure 3.18 Line Graph of the Shear Stress that Occurs Along the Inner Edge

of the Vessel in the Aneurysmal Vessel 49

Figure 3.19 Red Line Indicating the Cut Line from Which the Following Data was

Extracted 50

Figure 3.20 Line Graph of the Velocity Across the Centerline of the Aneurysm 51

Figure 3.21 Line Graph of the Shear Stress Across the Centerline of the Aneurysm ....51

Figure 3.22 Particle Tracking Simulation at 1 Second after Initial Particle Release ...... 52

Figure 3.23 Velocity $(\mathrm{m} / \mathrm{s})$ Comparison of the Experimental Findings and the

COMSOL MULTIPHYSICS $®$ Findings .53

Figure 4.1 A Range of Shear Stress Magnitudes encountered in veins, arteries, and in low-shear and high-shear pathologic states .56 


\section{INTRODUCTION}

Aims of this Thesis:

Aim I: To validate a COMSOL MULTIPHYSICS $®$ model of a full iliac artery aneurysm with an empirical model.

Aim II: To create a 3D dimensionally accurate model of a iliac artery aneurysm using additive manufacturing techniques for the purpose of clinical testing.

Aim III: To simulate a model in COMSOL MULTIPHYSICS $®$ comparing the fluid dynamics of an aneurysmal iliac artery to a non-aneurysmal iliac artery.

Iliac artery aneurysms continue to be prevalent and a life threatening pathology. If left untreated, the aneurysm can increase and even rupture. The objective of this study was to create an empirical model that could accurately mimic the same fluid dynamic properties of an iliac aneurysm found in the human body and create an in silico model using the finite elements program, COMSOL MULTIPHYSICS ® Modeling Software.

\subsection{Anatomy, Etiology, and Pathophysiology}

\subsubsection{Anatomy}

The common iliac arteries, or CIAs, are the terminal branches of the aorta and originate at the fourth lumbar vertebra and run downward and laterally along the medial border of the psoa muscle [2].The CIA then divides into the internal and the external iliac artery. The internal iliac artery runs from the pelvis to the upper border of the greater sciatic foramen where it will then further divide into anterior and posterior branches. The external iliac artery runs along the medial border of the psoa muscle, following the pelvic brim. Most iliac artery aneurysms, (>70\%), involve the CIA and $20 \%$ principally affect the 
internal artery [2]. For reasons still unknown, the external iliac artery aneurysm is very rare. Aneurysms can develop in two different forms, saccular and fusiform, see Figure 1.1.

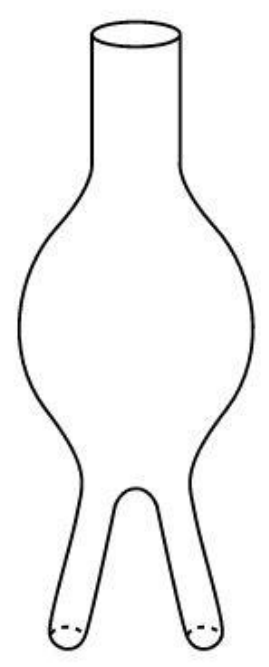

Fusiform

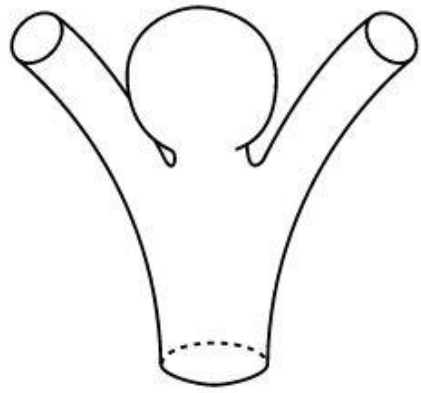

Saccular

Figure 1.1: Aneurysm Classification Based on the Form

The shape of the aneurysm plays an important role in development pathways, pressure gradients, and treatment methods. Saccular aneurysms are much easier to treat because the can be surgically removed or embolized without effecting blood flow. This topic will be further discussed in the treatments section. The most important part of the anatomy with regards to the development of aneurysms is physiological makeup of the wall of the vessel. All arteries are made up of three layers. The thick outermost layer, known as the tunica adventitia is made of connective tissue. The middle arterial layer, known as the tunica media is thicker and contains more contractile tissue. It consists of circularly arranged elastic fibers, connective tissue, and polysaccharide substances [3]. The final and thinnest layer is the tunica intima, located in the innermost position [3]. It is a single layer of simple squamous endothelial cells glued by a polysaccharide intercellular matrix. It is surrounded by a thin layer of subendothelial 
connective tissue interlaced with a number of circularly arranged elastic bands called the internal elastic lamina [3]

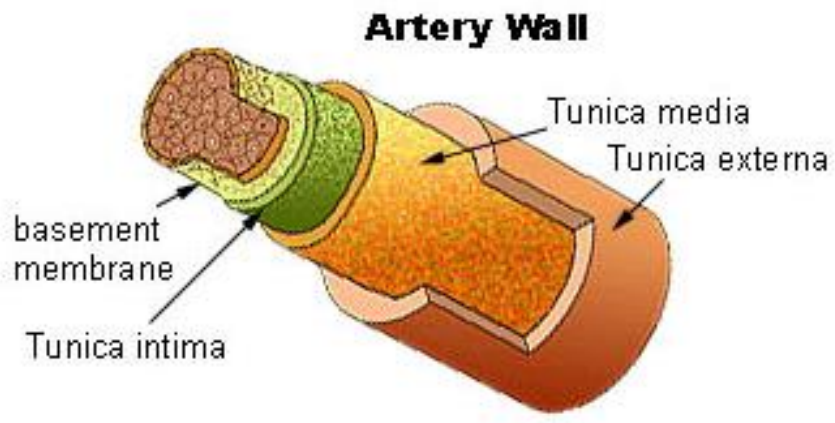

Figure 1.2: Physiologic depiction of an artery wall [3]

These layers each serve unique purposes to deal with the pressure from the heart. Arteries closer to the heart, like the aorta, have more elastin compared to arteries further away like the cerebral arteries [4]. The smooth muscle layer is usually reduced or disorganized at bifurcations as one vessel splits into two [4]. This restructuring makes bifurcations weaker and more susceptible to damage with changes in pressure, shear stress, and flow rates. Deficiencies in smooth muscle cells and collagen, which provides tensile strength, along the artery wall can be considered a risk factor for the development of aneurysms.

\subsubsection{Epidemiology}

lliac aneurysms are commonly found to be associated with aortic aneurysms; coexisting in approximately $10 \%$ to $20 \%$ of cases [5], aneurysms that are isolated in just the iliac artery are much more rare. lliac artery aneurysms are more commonly found in elderly men, men having preponderance (5:1) [6]. Aneurysms have the highest prevalence for people in their 70's and 80's [6]. 


\subsubsection{Pathogenesis}

The manner of development of iliac aneurysms is still not well known. It is believed to have a pathogenesis similar to that of aortic aneurysms, which is predominantly a degenerative process that can include proteolytic degradation of the aortic wall connective tissue, fluid dynamics, inflammation, and biomechanical wall stress [7]. The flow of blood, engenders on the luminal vessel wall and endothelial surface a frictional force per unit area known as hemodynamic shear stress [8]. At shear rates larger than $200 \mathrm{~Hz}$, the magnitude of the wall shear stress, $\tau_{w}$, can be estimated in most of the vasculature by Poiseuille's law (Equation 1.1),

$$
\tau_{w}=\frac{4 \mu Q}{\pi R^{3}}
$$

Where $\mu$ is the viscosity, $Q$ is the volumetric flow rate, and $R$ is the internal radius. This law states that shear stress is proportional to blood flow viscosity, and inversely proportional to the third power of the internal radius [9]. Figure 1.3 shows how different risk factors and mechanical factors such as hemodynamic stresses can contribute to a physiological process.

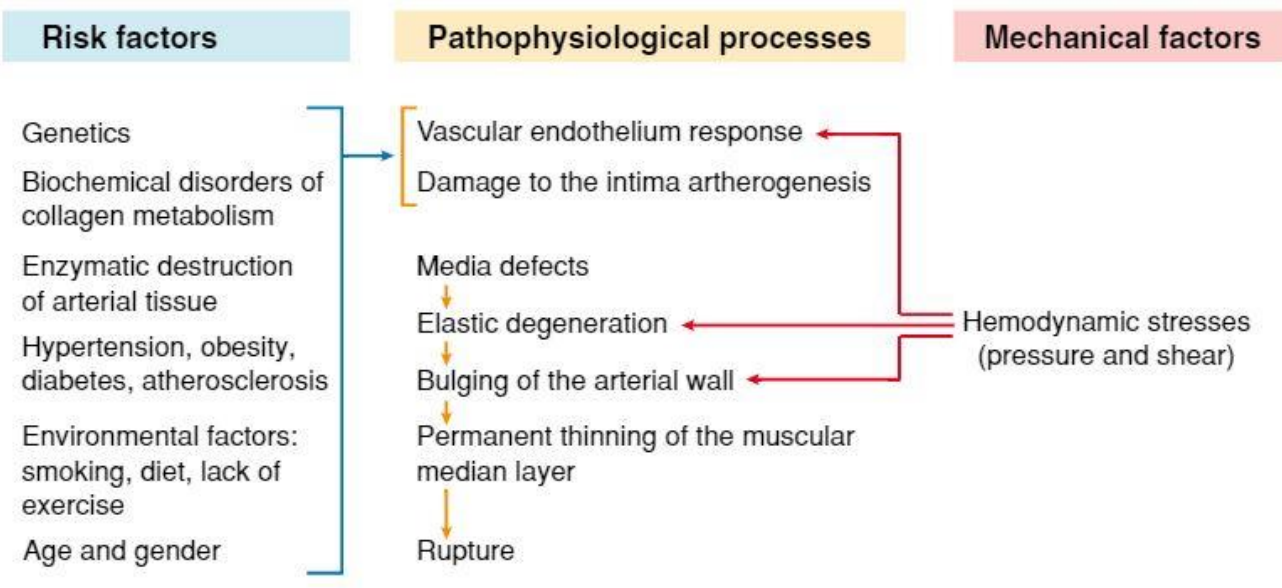

Figure 1.3: How Mechanical Factors and Risk Factors play a role in the pathophysiological process 
This figure illustrates that a combination of risk factors that might make one predisposed to an aneurysm and the added mechanical hemodynamic stress can cause a number of physiologic reactions, mainly the endothelium bioreceptor response, to trigger the bulging and rupture of the wall.

\subsection{Diagnosis}

\subsubsection{Imaging}

Unfortunately the diagnosis of iliac artery aneurysms often comes too late. Many aneurysms are asymptomatic until rupture, at which point it is too late for treatment. Fortunately, aneurysms are easy to diagnose if proper imaging can be performed. There are three main imaging techniques that allow for a proper diagnosis of iliac artery aneurysms: MRI, CT, and ultrasound. MRIs, which use nuclear resonance, provide the best resolution for soft tissue components of the body. By adding contrast, everything in the vasculature can be seen. However, MRls are expensive and limited, therefore may seem improbable for a diagnosis of an ailment that is asymptomatic. The contrast agent used in MRl's can also be dangerous for people with kidney problems. CT scans use Xray imaging, which is generally used for fractured bones but can still provide imaging of aneurysms. This technique is less expensive than MRls which means they are performed more frequently and in many cases provide diagnosis. Ultra-sound imaging, which uses sound waves, is one of the least expensive and least invasive methods of imaging of the methods discussed. All of these techniques can, and are used to diagnose iliac aneurysms, each with their own pros and cons. 


\subsubsection{Classification Scheme for lliac Artery Aneurysms}

In attempt to better organize the different diagnostic and treatment techniques used for iliac aneurysms, or IAAs, Sakamoto et. al [10] developed a classification scheme that will be employed to help categorize iliac artery aneurysms based on the anatomic features of the aneurysm and the relevant endovascular treatment option. The typical iliac vascular follows an anatomy depicted in figure 1.4

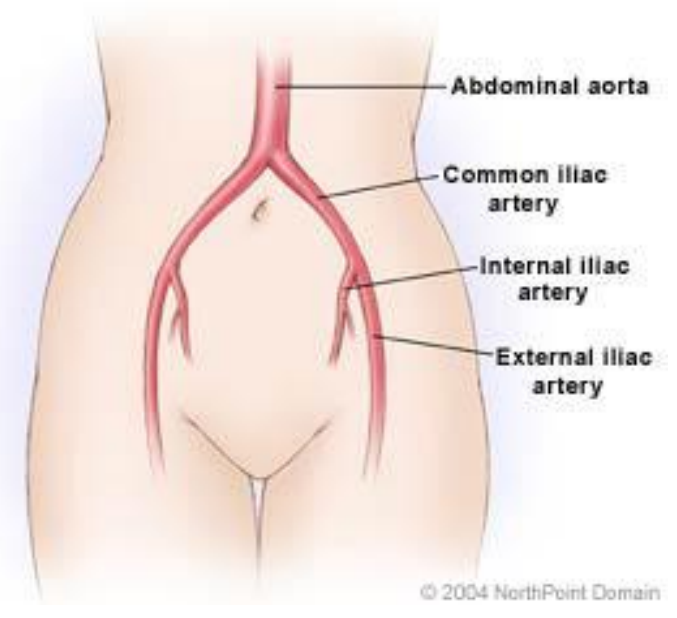

Figure 1.4: Typical iliac artery

Type I IAAs are internal IAAs that are far enough $(>2 \mathrm{~cm})$ from the aortoiliac bifurcation of the internal iliac artery to allow proximal embolization treatment, whereas type II IAAs are internal IAAs that are not sufficiently distant to allow this procedure. Type III IAAs are common IAAs that are far enough from the aortoiliac bifurcation to allow placement of a straight stent-graft, whereas type IV IAAs are common IAAs that are not sufficiently distant to allow such placement. Type V IAAs are common or internal IAAs that develop after AAA repair with a bifurcated graft.

\subsection{Treatment}

Aneurysms less than $10 \mathrm{~mm}$ are often treated with medication or simply monitored for growth, but aneurysms over $10 \mathrm{~mm}$ in diameter often require surgical removal [11]. 
Specifically, if an aneurysm has ruptured or is growing at a rate of $0.90 \mathrm{~mm}$ or greater per year, surgical removal is recommended regardless of size. Unlike saccular aneurysms, fusiform aneurysms cannot be clipped or removed. Due to their position within the vasculature, the parent artery can still play a large role in bodily function. This poses a challenging question, how to treat the aneurysm without blocking fluid flow. Fortunately, unlike aortic artery aneurysms, CIAs occur in vasculature that can easily enable bypass surgery and the diseased portion can be isolated using a number of different techniques outlined below.

\subsubsection{Type I}

Type I IAAs can be treated using two different methods as graphically depicted in Figure 1.5

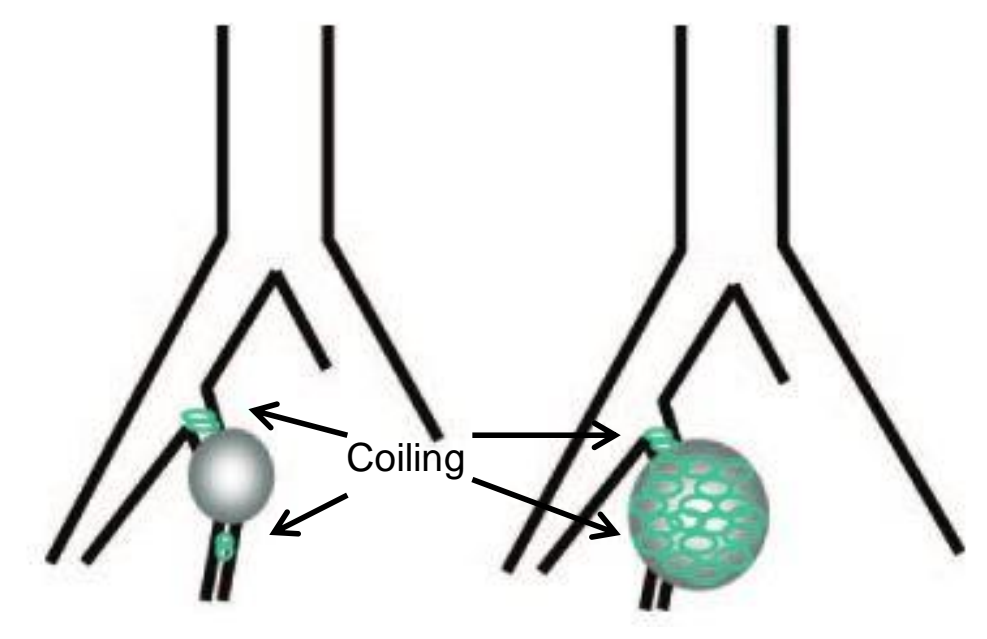

Figure 1.5: Treatment Process for a Type I IAAwith a stent-graft (left) and a bare stent (right)

The proximal and distal embolization method which uses a coiling material to cut of circulation from the proximal and distal ends of the aneurysm. This is one of the least invasive methods and can be used on Type IIAAs because the aneurysm is far enough 
from the main of the internal iliac artery. The second treatment method that can be used for Type I IAAs is coil packing and proximal embolization. Coil Packing is one of the more popular methods of treatment but can be hard to perform on fusiform aneurysms. Coil packing consists of tiny coils being packed into the aneurysm to promote blood clotting and close off the aneurysm [10].

\subsubsection{Type II}

Type II IAAs can be treated using two different methods graphically depicted in Figure 1.6 .

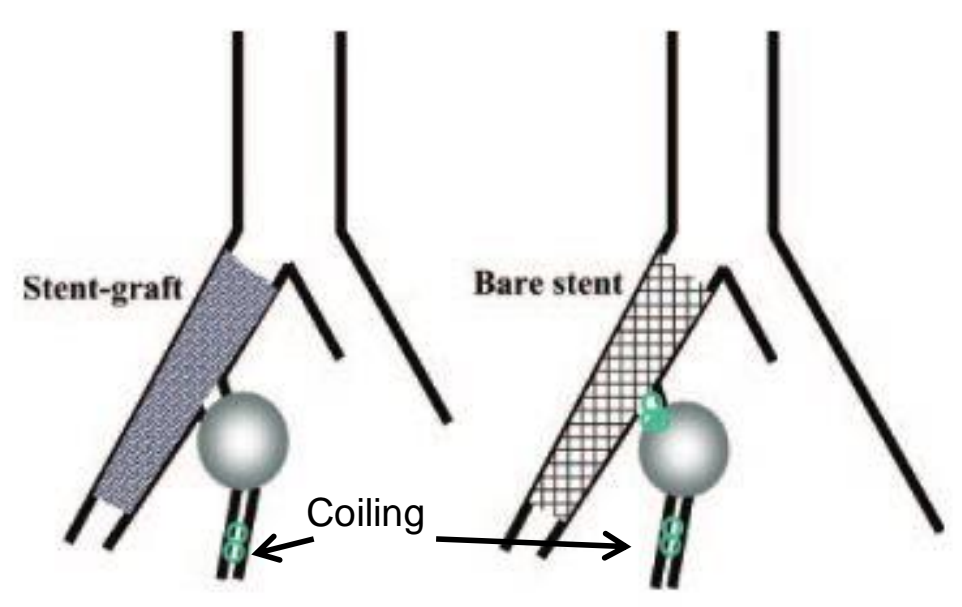

Figure 1.6: Treatment Process for a Type II IAA

The process on the left showing proximal and distal coiling and the process on the right showing coil packing and proximal embolization

Treatment for type II IAAs are considered when internal IAAs are less than $2 \mathrm{~cm}$ from the origin of the internal iliac artery. Stent-graft placement across the origin of the IAA is functionally equivalent to proximal embolization. Therefore, distal embolization of the aneurysm followed by stent-graft placement is thought to be an effective alternative treatment. Distal embolization of the aneurysm, placement of a bare stent across the origin of the IAA, and proximal embolization through the wire mesh of the stent are 
possible alternatives for when the common or external iliac are torturous or too small. The bare stent is positioned to prevent migration of coils into the common or external iliac artery [10].

\subsubsection{Type III}

Treatment for type III IAAs is graphically depicted in Figure 1.7.

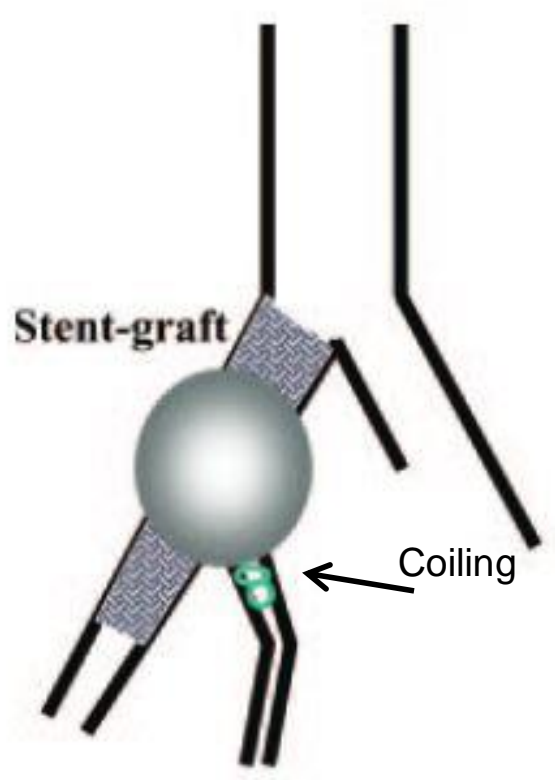

Figure 1.7: Treatment Process for a Type III IAA Stent-graft placement as well as distal embolization

Common IAAs that are more than $2 \mathrm{~cm}$ from the aortoiliac bifurcation can be treated with stent-graft placement in the ipsilateral common and external iliac arteries [10]. This technique must be performed with a distal embolization to ensure that retrograde flow to the aneurysm does not occur [10]. 


\subsubsection{Type IV}

Treatments for type IV IAAs are graphically depicted in Figure 1.8.
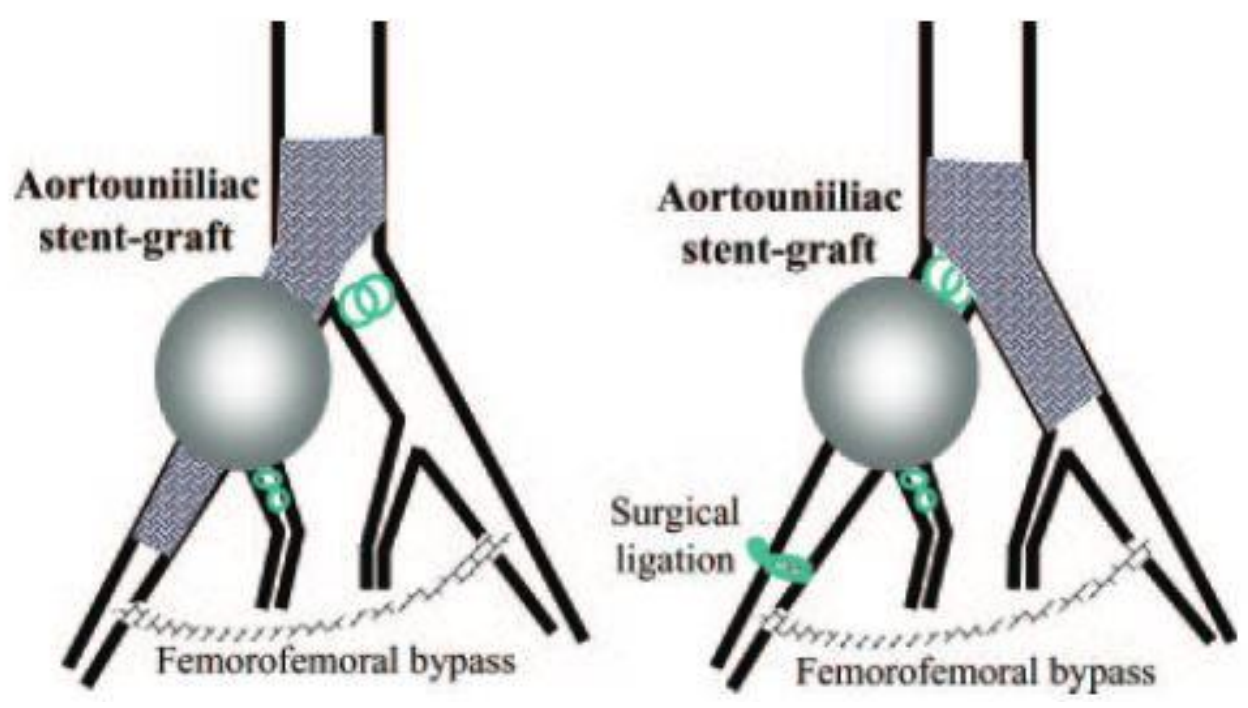

Figure 1.8: Treatment Process for a Type IV IAA Stent-graft placement in the aorta and a single iliac artery

Common IAAs that are less than $2 \mathrm{~cm}$ from the aortoiliac bifurcation can be challenging to treat with stent-grafts because of the high risk of endoleaks[10], which result from incomplete sealing of the stent-graft at the proximal attachment site[10]. As an alternative, type IV common IAAs can be treated with stent-graft placement in the aorta and an iliac artery. Generally, stent-graft placement in the aorta and an ipsilateral iliac artery is selected as a first-line option[10]. Stent-graft placement in the aorta and a contralateral iliac artery is advised when the ipsilateral iliac artery is extremely tortuous or too small. In addition, bifurcated aortoiliac stent-graft placement is an alternative for type IV aneurysms. However, this technique necessitates sufficient diameter of the lower abdominal aorta for placement of the bifurcated graft and sufficient diameter of the iliac arteries for insertion of the large delivery devices [10]. 


\subsubsection{Type V}

The final and most complex to repair aneurysm, type $V$, is graphically depicted in Figure 1.9.

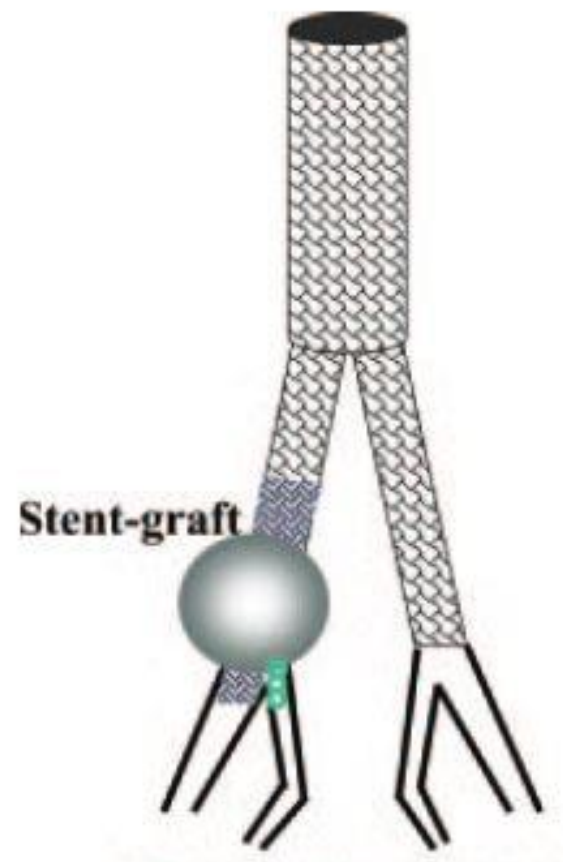

End-to-end anastomosis

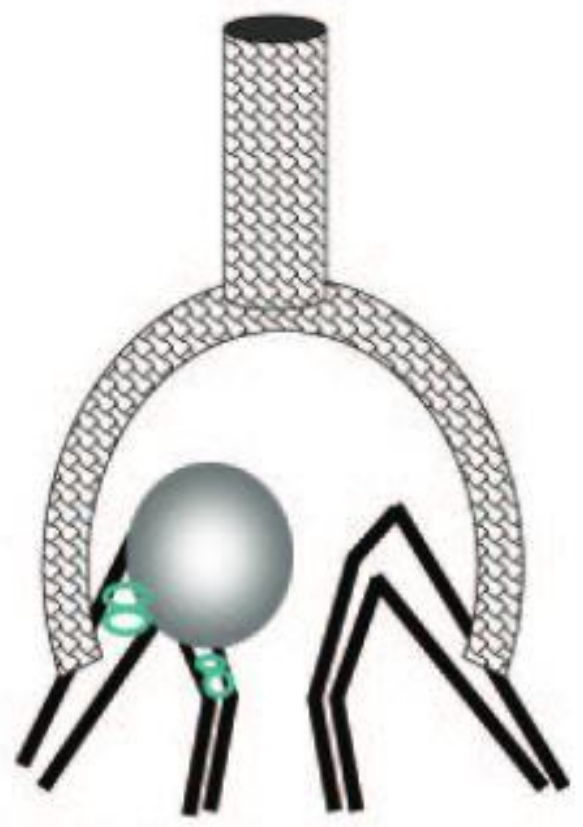

End-to-side anastomosis with the external iliac artery

Figure 1.9: Treatment Process for a Type V IAA

Common or internal IAA that develops after AAA repair with a bifurcated graft

When the common iliac arteries are affected with aneurysmal or severe atherosclerotic change, the graft limbs may be anastomosed (the reconnection of two flow paths that previously branched out) or placed end-to-side with the external iliac or femoral arteries, followed by over-sewing of the orifice of the common iliac arteries [10]. This surgical procedure can help preserve retrograde flow to the internal iliac arteries and prevent ischemia of pelvic organs [10]. 


\subsection{Previous Fluid Flow Research}

lliac aneurysms are extremely rare, occurring in about 6.58 men in a 100,000 population in the united states [12] making them difficult to research. Most research on aneurysms has been done on the intracranial aneurysm and the abdominal aortic aneurysm due to their higher prevalence and rate of fatality [12]. However, there has been a general lack of research done on aneurysms because there are essentially undetectable until rupture.

\subsubsection{Mechanical Properties of lliac Aneurysms}

Healthy shear stresses on the luminal wall of the artery, owing to normal blood, are normal in the human body. Measurements using different modalities show that shear stress ranges from 1 to $6 \mathrm{dyne} / \mathrm{cm}^{2}$ on the venous side and between 10 and 70 dyne $/ \mathrm{cm}^{2}$ in the arterial side; high shear thrombosis occurs anywhere after $70 \mathrm{dyne} / \mathrm{cm}^{2}$ [11]. Shear stress in the vessel is controlled by the endothelial cells that line the vessel. The endothelial cells have mechanoreceptors that allow them to sense the shear stress due to flow of blood over their surface. By signaling this information to the surrounding cells, they enable the blood vessel to adapt it's diameter and wall thickness to suit the blood flow [13].

The past three decades have provided numerous hypotheses including the widely accepted hypothesis that specific changes in the hemodynamic forces acting on the vessel wall could be a key contributing factor to the origin and progression of aneurysms [11] [14]. If these changes in hemodynamic forces can be characterized and modeled, researchers can develop a better understanding, leading to more accurate diagnosis and treatment. There have been many studies analyzing the behavior of blood flow in the body and how that behavior effects the development of aneurysms. One study suggests that in the portion of large elastic arteries located upstream of a bifurcation, such as the abdominal aorta, and the iliac, femoral, and popliteal arteries, 
the amplitude of the pressure wave (pulse) is considerably modified as a result of the reflection of the wave in the bifurcation [8]. This modification can result in an amplification of the pressure waves leading to an increased likelihood of the development and progression of an aneurysm.

\subsubsection{Progression and Enlargement Rate}

It seems to follow that the same factors that lead to the formation of the aneurysm can be responsible for the enlargement process. The flow shear endothelium-mediated initiation hypothesis [15] has prompted many fluid mechanics studies aimed at determining the wall shear stresses of abdominal aortic aneurysms, or AAAs, at different stages of their development. These studies are useful for studying iliac artery aneurysms because both the AAA and iliac artery aneurysms typically take the fusiform shape (Figure 1.1). These studies have consisted mainly of experiments and numerical simulations utilizing ideal symmetric and non-symmetric shapes of fusiform aneurysms and in realistic geometries reconstructed from three-dimensional volume rendering of high-resolution CT scans and angiographies. Figure 1.10 shows one result from a study which showed flow conditions near the walls during the deceleration portion of the cardiac cycle, a relatively coherent array of large vortices form and the blood flow slowly recirculates [16][17]. Figure 1.10 shows Digital particle image velocimetry (DPIV) measurements of the instantaneous velocity field, instantaneous streamlines, and shear stress field in a symmetric model of an abdominal aortic aneurysm (AAA) fusiform aneurysm. 


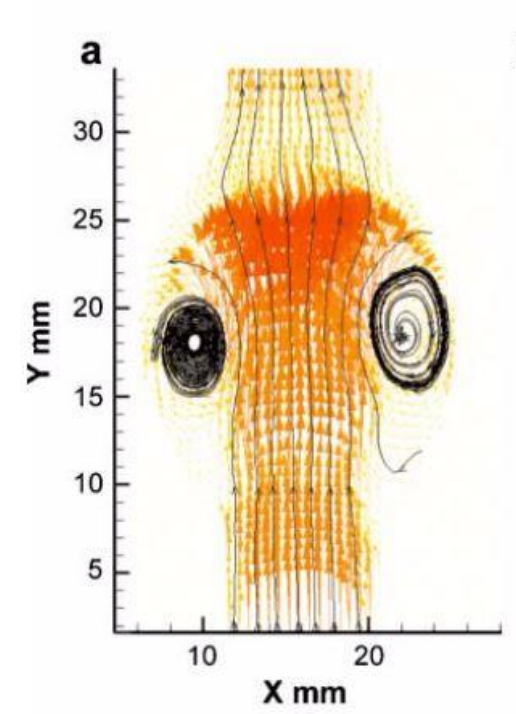

High shear stresses: activation mechanisms
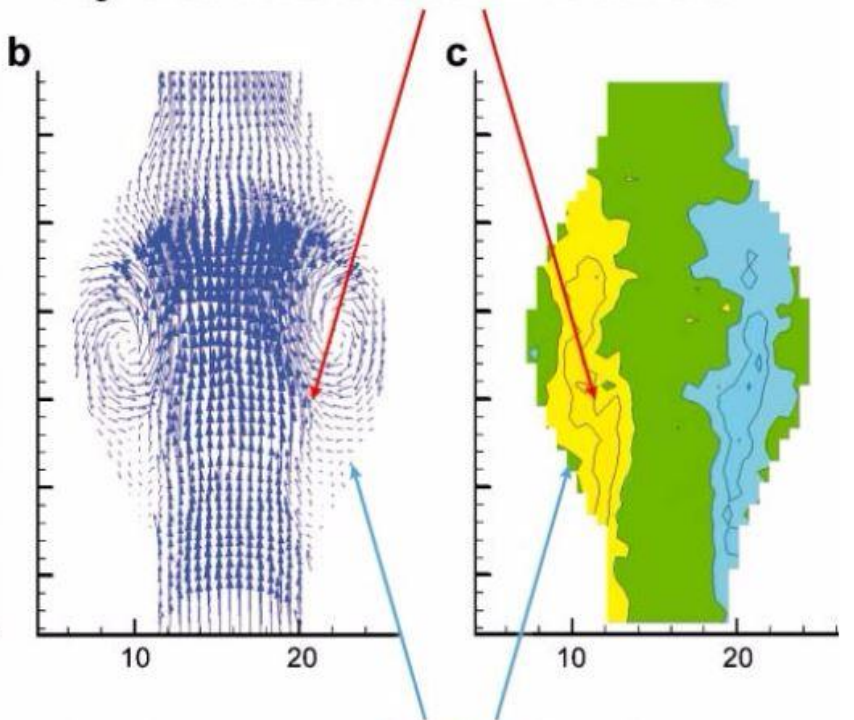

Low shear stresses: thrombus formation

Figure 1.10: Digital particle image velocimetry(DPIV) measurements of the (a) instantaneous velocity field, (b) instantaneous streamlines, and(c) shear stress field in a symmetric model of an abdominal aortic aneurysm (AAA) fusiform aneurysm. The measurements correspond to the beginning of the deceleration after peak systole

This study demonstrated that in the low shear stress regions of the aneurysm, the proximal sides, thrombus formation occurred as a result of the remodeling mechanism of the endothelial cells. Studies have also shown that as aneurysms grow nonsymmetrically, the location and magnitude of the regions of high gradients of temporal and spatial shear stresses, as well as the extent of the regions of low but oscillatory shear stresses along the wall, vary significantly[18][19].

\subsubsection{Previous Study by Author}

In a study previously performed by the author, a half vessel of the iliac artery aneurysm was created empirically using similar manufacturing techniques. The previous study also successfully verified the accuracy of the COMSOL MULTIPHYSICS ® simulation with a half vessel empirical model. This study will work to add a comparative element to the COMSOL MULTIPHYSICS $®$ simulation by running tests on a non-aneurysmal vessel, 
and create a full vessel empirical model that can be used to deploy stents and other treatment technologies. 


\section{METHODS AND MATERIALS}

\subsection{A Background on Experimental Techniques That Were Used}

\subsubsection{Fused Deposition Modeling}

Rapid Prototyping (RP) has become an essential tool in the enginee ring world. In recent years, RP has allowed for the successful build of physical models faster and with more complex geometries. Fused Deposition Modeling, or FDM, has become one of the most popular and successful rapid prototyping techniques. The Fused Deposition Modeling process was originally developed by Stratasys [20], which horizontally deposits molten thermoplastic material, such as ABS and PLA (PLA was not used for this study), extruded from a nozzle (figure 2.1).

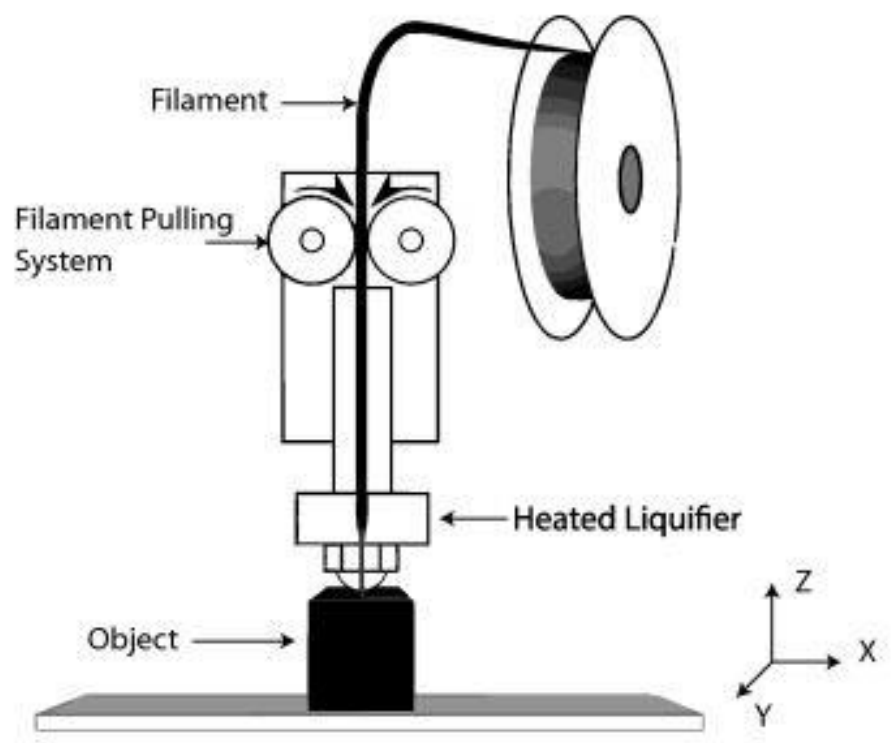

Figure 2.1: Fused Deposition Modeling Process 
The filament is deposited layer-by-layer based on the 3D CAD data. A filament is softened and melted inside the liquefier, and is extruded through the nozzle by the still solid upstream filament. As the liquefier moves, the extruded polymer is laid down, starting with the object perimeters and then the filling, for each layer of the object [20]. One key material property that makes FDM so valuable is that unlike other RP techniques the material properties of FDM do not change with time or environmental exposure [21]. The materials maintain their strength, color, and toughness in almost any environment. The biggest limitation, acknowledged by both users and Stratasys, is surface finish. Due to the extrusion process of the semi-molten plastic, FDM exhibits a rough finish [21]. The finished product will still show contours of the passes of the extrusion tip and the build layers [21]. This problem can be mitigated by using lacquers to fill in any contours or by using sanding techniques.

\subsubsection{Plasma Bonding}

Plasma bonding falls under the fabrication classification of soft lithography. The use of plasma bonding in this study involved plasma bonding PDMS to a glass slide. PDMS is a synthetic polymer (silicone rubber), as the name implies, it has a SiOSi backbone with each Si atom having two methyl groups $(\mathrm{CH} 3)$, this is what makes PDMS a good candidate for plasma bonding. In the case of plasma bonding PDMS to glass, it is considered an irreversible bonding technique in which the exposure to plasma is thought to create $\mathrm{OH}$ groups on surface of the PDMS and glass. These revert to SiOSi bonds when the surfaces are brought together. The bond can withstand applied pressures up to $30 / 50$ psi [22]. 


\subsubsection{PDMS Bonding}

PDMS bonding is commonly used to seal and combine two PDMS structures. A number of PDMS bonding techniques have been reported in the literature over the last several years as the focus on multilayer PDMS microfluidic devices has increased [23]. Oxygen plasma bonding as outlined in section 2.1.2, despite cost, additional fabrication time and inconsistent bonding results, has remained a widely used method for bonding PDMS layers and is considered the gold standard for PDMS bonding. A comparative study by M.A Eddings, et. al, of four rapid, inexpensive alternative PDMS-PDMS bonding approaches was undertaken to determine relative bond strength. These include corona discharge, partial curing, cross-linker variation and uncured PDMS adhesive. Partial curing and uncured PDMS adhesive demonstrated a considerable improvement in bond strength and consistency by retaining average bond strengths of over $600 \mathrm{kPa}$, which was more than double the average bond strength of oxygen plasma. PDMS bonding is a much more simple method that does not require a clean room or oven for its process. It also forgoes the need for expensive equipment and training. In a previous study conducted by the author that analyzed half of an iliac artery aneurysm, plasma bonding was used to bond the PDMS construct to glass. For this full vessel, where PDMS must be bonded to PDMS, the author has decided to use the PDMS bonding technique after reviewing the success of the study by M.A Eddings et. al.

\subsubsection{Image J}

Image $\mathrm{J}$ is an open source image processing program designed for scientific multidimensional images. ImageJ can be used for many different purposes including automatic cell counting, measuring cell traction forces, ion beam imaging, and axonal growth analysis, to name a few. For the purposes of this study, ImageJ was used to analyze the velocity of fluid flow through a tube (the vessel). Image J was able to set 
threshold boundaries for color and contrast allowing for a more accurate calculation and was also able to split a 32-bit color image into RGB components allowing for a more simplified analysis.

\subsection{Materials Used}

The materials used for this study are summarized in table 2.1

Table 2.1 List of materials

\begin{tabular}{c|c|c|}
\hline \hline \multicolumn{2}{c}{ Material } & Panufacturer \\
\hline \hline $\begin{array}{c}\text { Acrylonitrile Butadiene } \\
\text { Styrene (ABS) 1.75mm } \\
\text { Filament }\end{array}$ & Hatchbox & 3D ABS-1KG1.75-BLK \\
\hline $\begin{array}{c}\text { Sylgard 184 Silicone } \\
\text { Elastomer Kit } \\
\text { (Polydimethylsiloxane } \\
\text { (PDMS)) }\end{array}$ & Dow Corning & 184 SIL ELAST KIT 0.5KG \\
\hline Glass (Glass Slide) & & N/A \\
\hline $\begin{array}{c}\text { Silicone Based Epoxy } \\
\text { Caulk }\end{array}$ & Loctite & LOCTITE® EPOXY \\
\hline INSTANT MIX ${ }^{\text {TM }}$ 5 MINUTE \\
\hline Water Mixed with food dye & ACE & 12589 082901125891 \\
\hline Acrylic Sheet & N/A & N/A \\
\hline $\begin{array}{c}\text { XTC-3D High Performance } \\
\text { 3D Print Coating }\end{array}$ & NTC-3D & N/A \\
\hline
\end{tabular}

\subsection{CAD Model}

The first step towards creating both the 3D vessel and the computer simulation vessel was designing a CAD model in SolidWorks. This process began by creating a skeleton of the blood vessels involved in the IAA. This step involved drawing a simple line roughly the length of the aorta. Inferior to the aorta, two branches were drawn using the line tool to represent the common iliac artery. Inferior to the common iliac artery, one more branch was constructed to the interior of the common iliac arteries in order to divide the common iliac artery into an interior and exterior iliac artery. The entrance length was calculated as $1.7 \mathrm{~cm}$ at minimum; however the entrance length was design as 
approximately $13 \mathrm{~cm}$. The dimensions for the lengths of the arteries distal to the aneurysm were arbitrary. This assumption was made because no data would be obtained distal to the aneurysm. At this point a rough skeleton of the vascular structure was complete. Next, the spline tool was used to create the organic shape of the vasculature. Points were then assigned at fixed distances along the skeleton of the vessel to act as place markers for the construction of the diameters of the vessel (Figure 2.2).

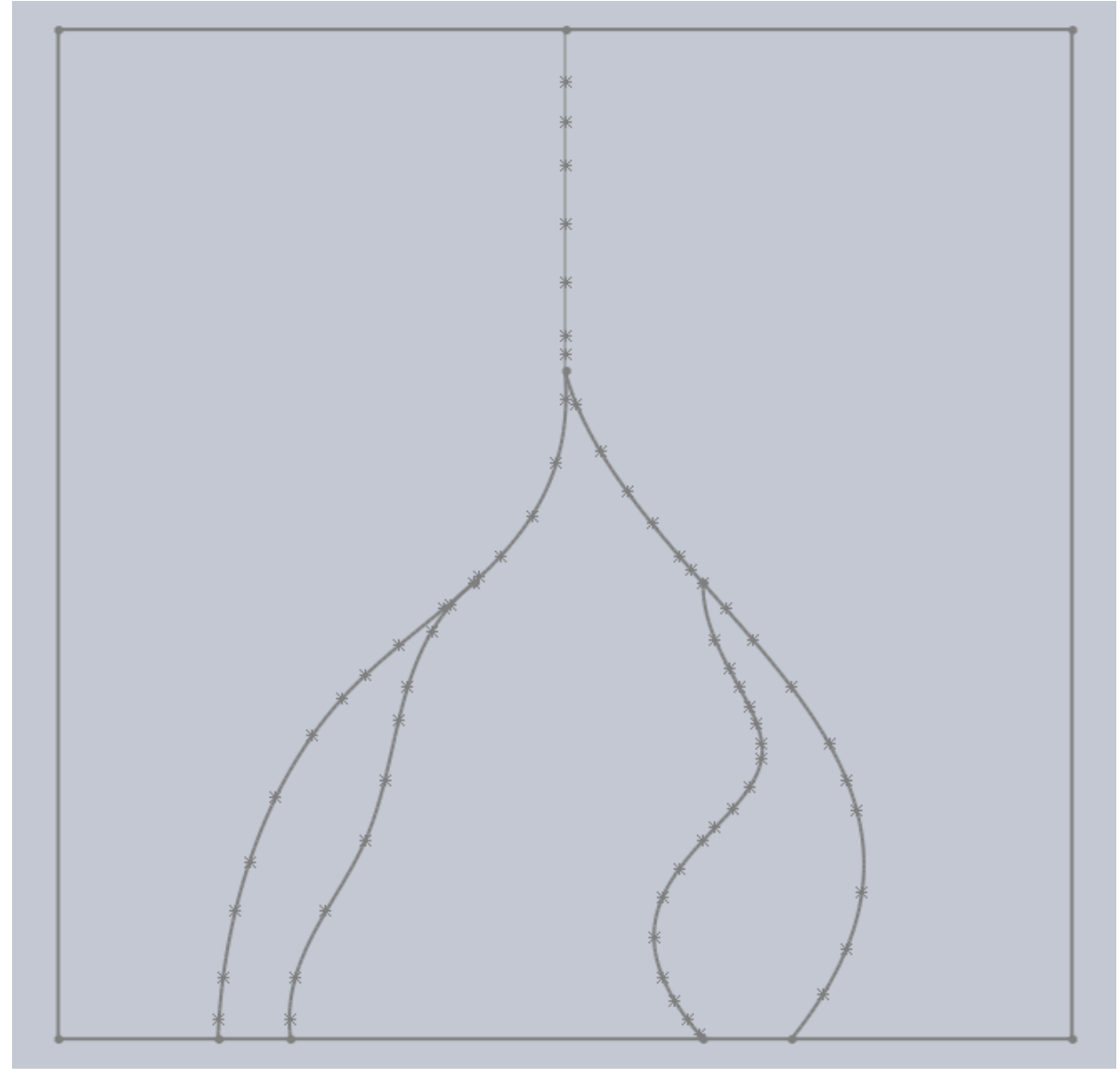

Figure 2.2: Skeleton of the Model with Points

Circles were then drawn at each of the points orthogonal to the spline to represent the widths of the vessels. The circle diameters increased as the location of the aneurysm approached (Figure 2.3). 


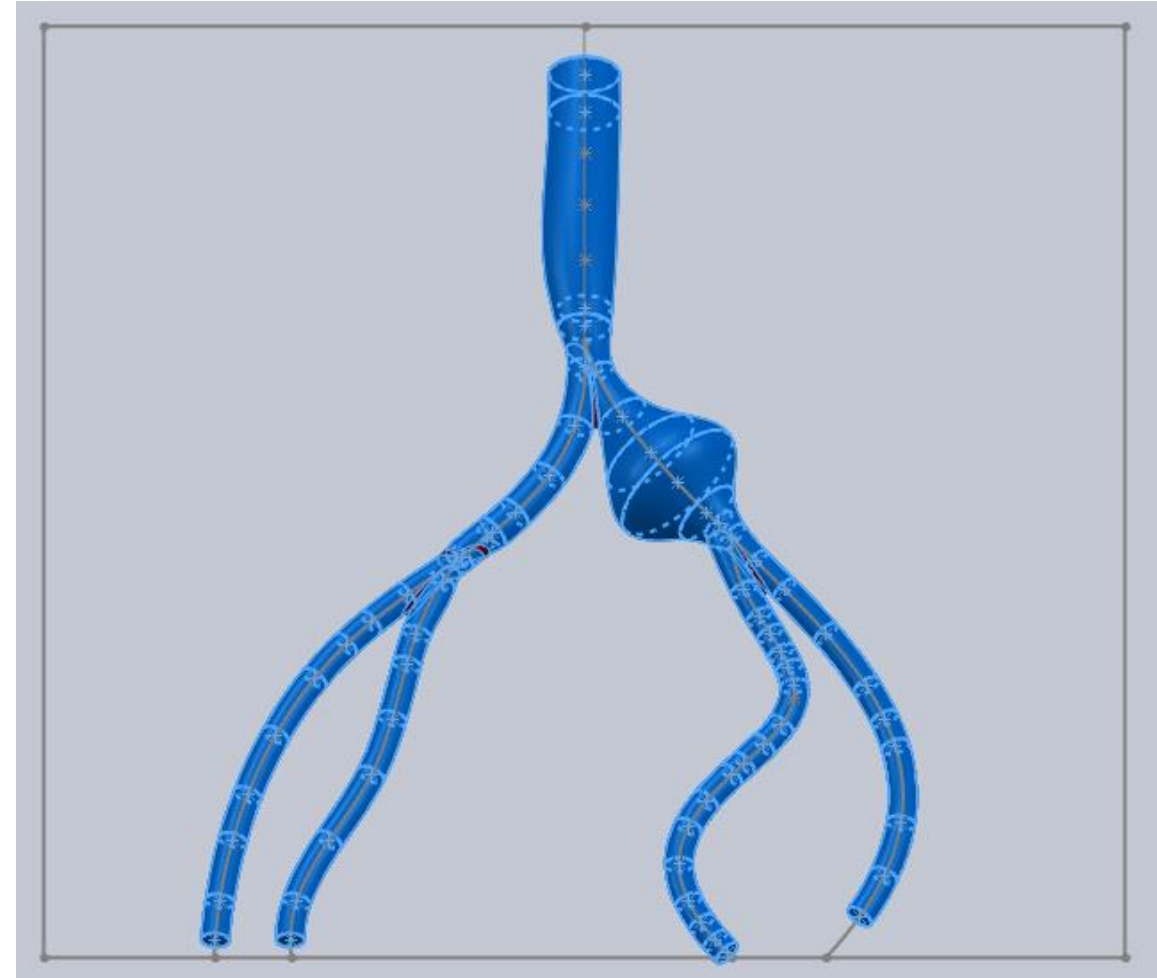

Figure 2.3: SolidWorks Part Showing the Circles used to Create Width

The dimensions for the circles were taken from ratios of multiple angiog rams with the maximum diameter of the aneurysm being $4 \mathrm{~cm}$. The lofting tool was then used to connect the circles to each other and create a solid part (Figure 2.4). 


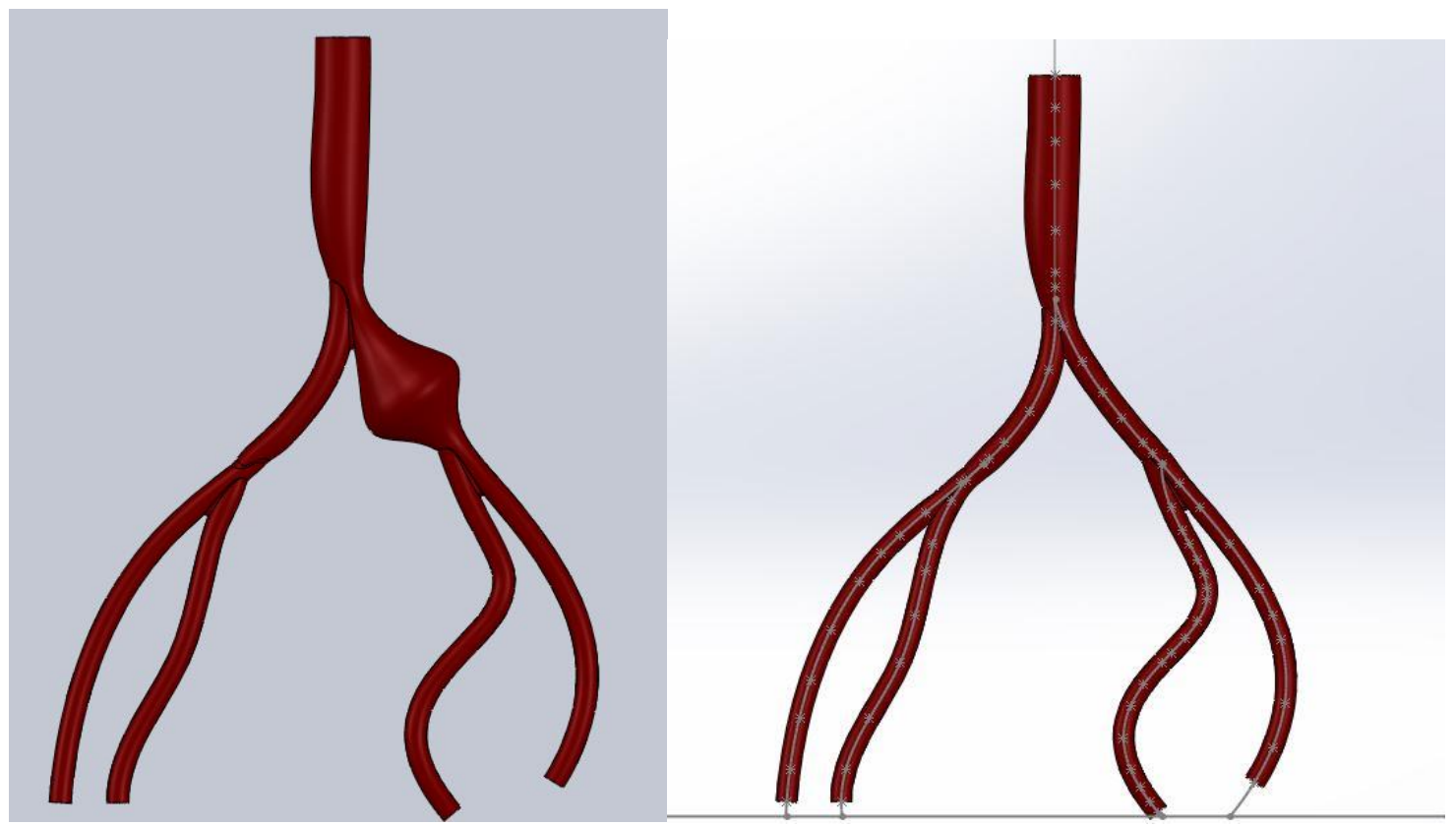

Figure 2.4: Lofted SolidWorks Part

Fillets were used at the bifurcations in order to round out the sharp edges that were results of the lofting process. This model was saved as the aneurysm model. A duplicate model had to be made with no aneurysms to compare the differences in a COMSOL MULTIPHYSICS $®$ study. The STL file of aneurysm model was modified in Solidworks by editing the diameters of the vessel at the aneurysm. The result was an identical CAD file with no aneurysm depicting a matching healthy common iliac artery. The STL files were then converted to IGES files to achieve a better quality import in COMSOL MULTIPHYSICS $\AA$. The IGES files were then opened in COMSOL MULTIPHYSICS $₫$ and the vessel was split in half along the coronal plane so that the printed vessel would lie along the glass slide (Figure 2.5). 


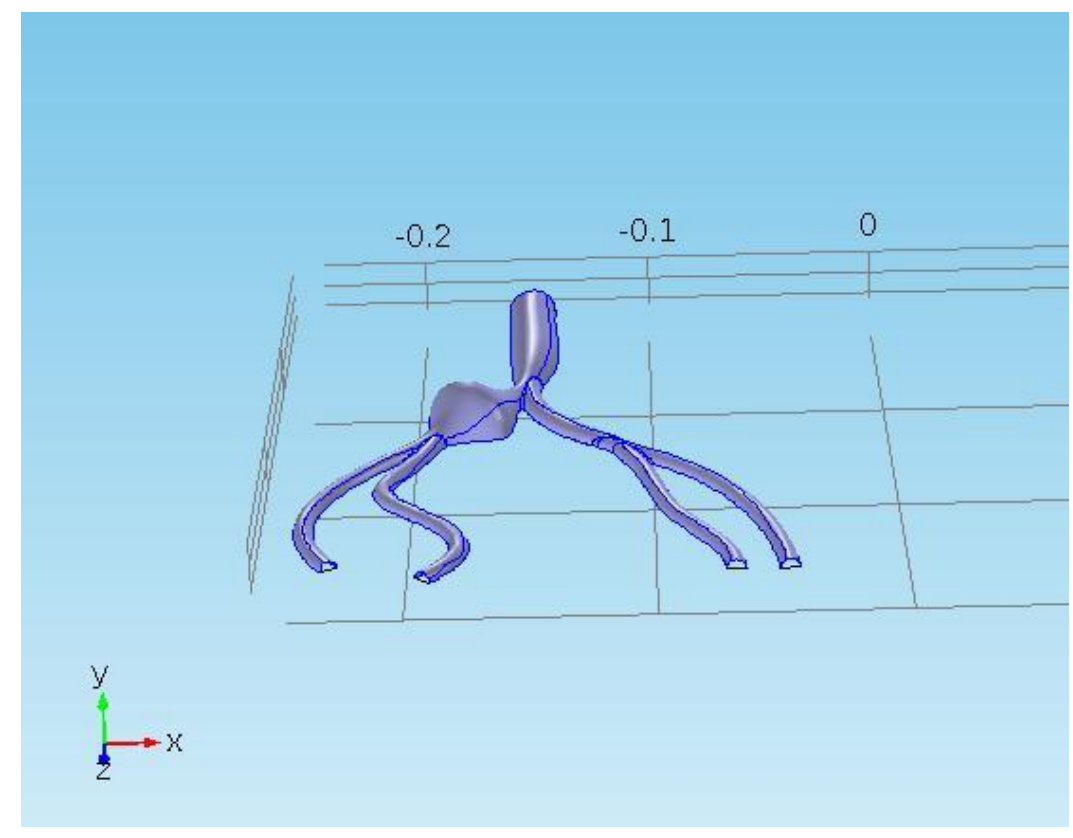

Figure 2.5: COMSOL MULTIPHYSICS $®$ Part Split along the Coronal Plane

Two half vessels were made so that the 3D printed part could be easily removed. In order to split the vessel, a block was created in COMSOL MULTIPHYSICS $®$ and was overlaid on half of the vessel. The block was then subtracted from the model and a half vessel was the result. This process was repeated using the other side of the COMSOL MULTIPHYSICS $\AA$ model to create a mirrored half vessel for printing, however only one half vessel was necessary for the COMSOL MULTIPHYSICS $®$ simulation due to symmetric capabilities of the software. This iteration of the model was saved for later simulations. The lack of build surfaces for FDM required that each CAD model be further split into three pieces for a total of six printed pieces. 


\subsection{Vessel Construct}

\subsubsection{FDM Construction}

The STL file of each individual piece was input into a slicer program (Slic3r with Repeyier-Host) which defined a path for the print head. The G-Code from the slicer was then exported to a USB drive. This information was then delivered to the Lulzbot Taz 5 for printing. The Lulzbot Taz 5 printer is a dual extruding desktop 3D printer owned and manufactured by the author. The filament was then loaded by attaching the filament wheel and pushing the filament through the extruder. The bed temperature was then set to $110^{\circ} \mathrm{C}$ and the extruder temperature was set to $220^{\circ} \mathrm{C}$. The printer then had to be calibrated to ensure the alignment of the axes. The vessels were printed with ABS using $30 \%$ infill and a $300 \mu \mathrm{m}$ layer thickness. The $30 \%$ infill on the print saved material costs and time but created more buoyancy which would lead to issues in the mold building phase. The separate pieces of the print were joined using a fast curing epoxy. The pieces were set aside to cure for 24 hours. After the epoxy had completely cured, a coating (XTC-3D High Performance 3D Print Coating) was applied to the printed part to help mitigate the consequences of the ridged layers. It works as a protective coating for smoothing and finishing 3D printed parts. Two liquids are mixed together and brushed onto the 3D print. The coating self-levels and wets out uniformly. The coating took only a few minutes to apply and was then left for four hours to cure. The result was a much smoother surface that helped minimize the ridges and protect the PDMS construct from accumulating any debris from the 3D printed part.

\subsubsection{Vessel Enclosure}

Three plexiglass walls of dimensions 3"X12" were attached to a gla ss slide using caulk. The walls and slide were cleaned using isopropyl alcohol. The triangle formation was an 
improvement made to the last iteration of the study to limit the amount of PDMS used and create a more portable construct.

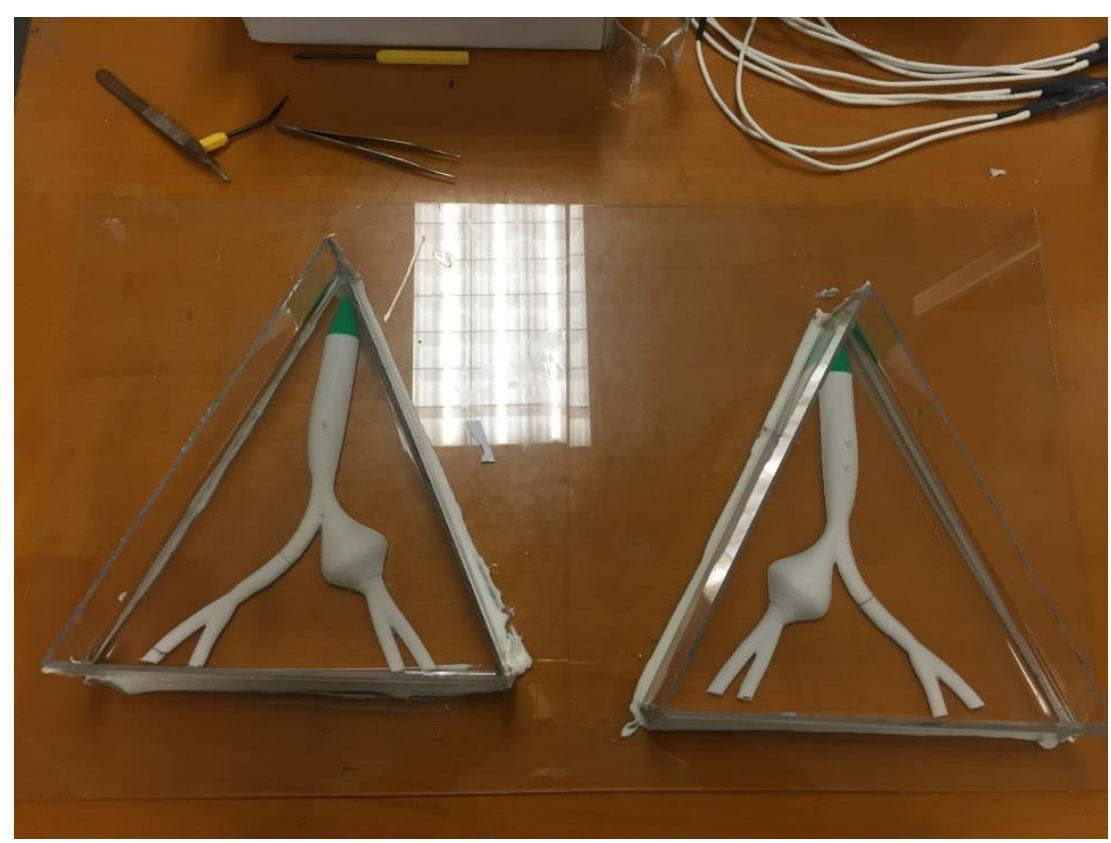

Figure 2.6: Vessel Enclosure

\subsubsection{PDMS Processing}

The PDMS was prepared by mixing the elastomer base with the curing agent for 10 minutes using a spatula with a 10:1 ratio of elastomer base to curing agent. The PDMS was then placed in a desiccator to remove the air bubbles (Figure 2.7). 


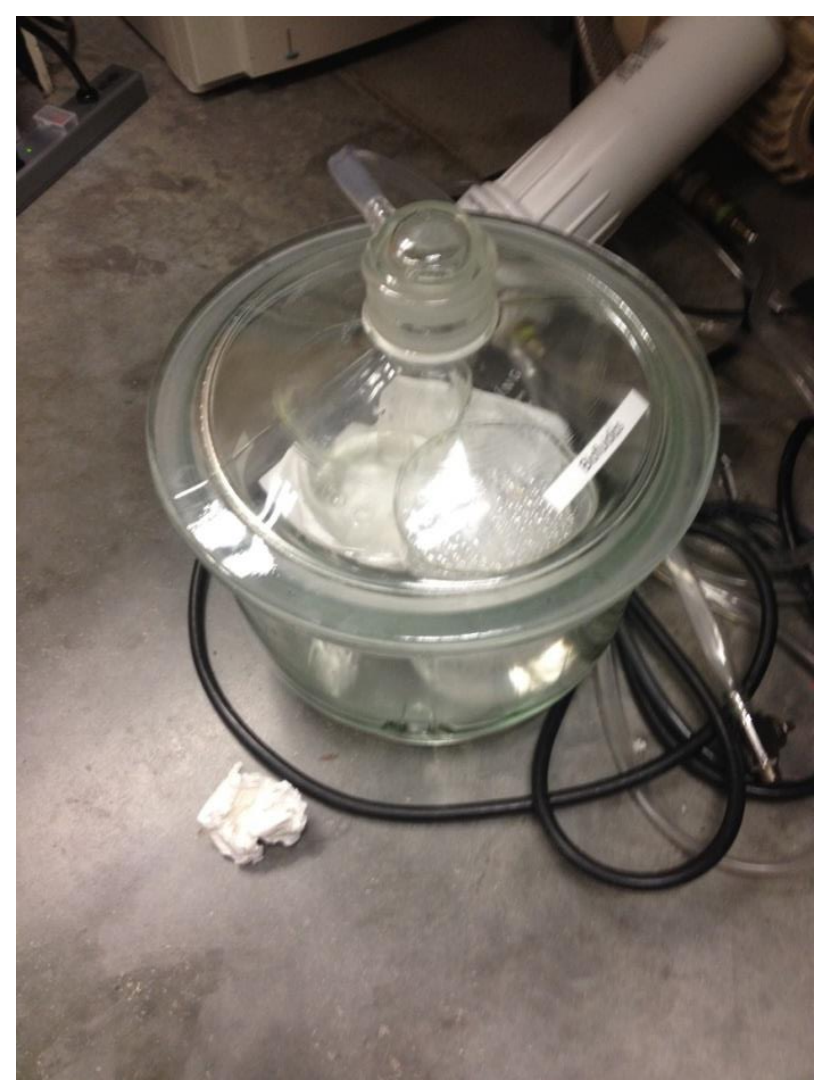

Figure 2.7: PDMS Process

The desiccator was pulsed until the air bubbles no longer over flowed the beaker of PDMS and was then left on until no air bubbles remained in the PDMS (approximately 1 hr). The printed aneurysm was assembled and placed in the vessel enclosure (Figure 2.6). Half of the prepared PDMS was then poured over both of the aneurysm constructs; however, due to the lack of infill, the model began to float. Forceps were used to stabilize the model while it was left to dry overnight. The forceps were placed on the aorta at around half the vessel length. The forceps were weighted down by balancing a box of weights on the top of the forceps. Once the PDMS was set, the forceps were removed and the remaining PDMS was desiccated and poured over the construct to seal the holes from the forceps and capture the entire vessel. The two constructs were then left to sit for another 48 hours to ensure the complete curing of the PDMS. The walls of 
the enclosure were carefully pried from the PDMS constructs. Then, the PDMS constructs were pried from the glass slide. This step ended up causing many conflicts because the printed part was not lying completely flush to the glass slide due to the lack of a uniform weighting process. This resulted in some minor tears in the PDMS and a need for outlining the printed part with a razor blade to cut out the printed part (figure 2.8). Finally the printed model was removed (figure 2.9).

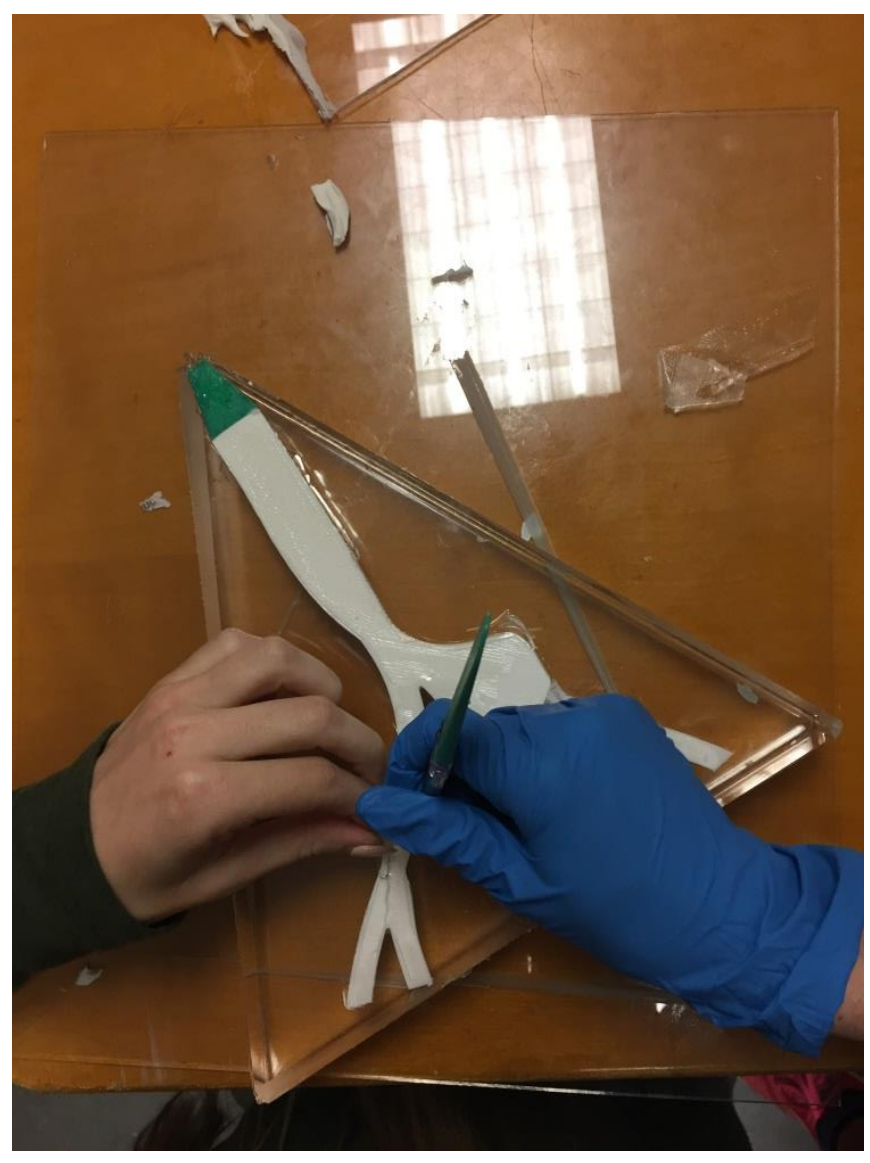

Figure 2.8: Removal of excess PDMS around the printed part 


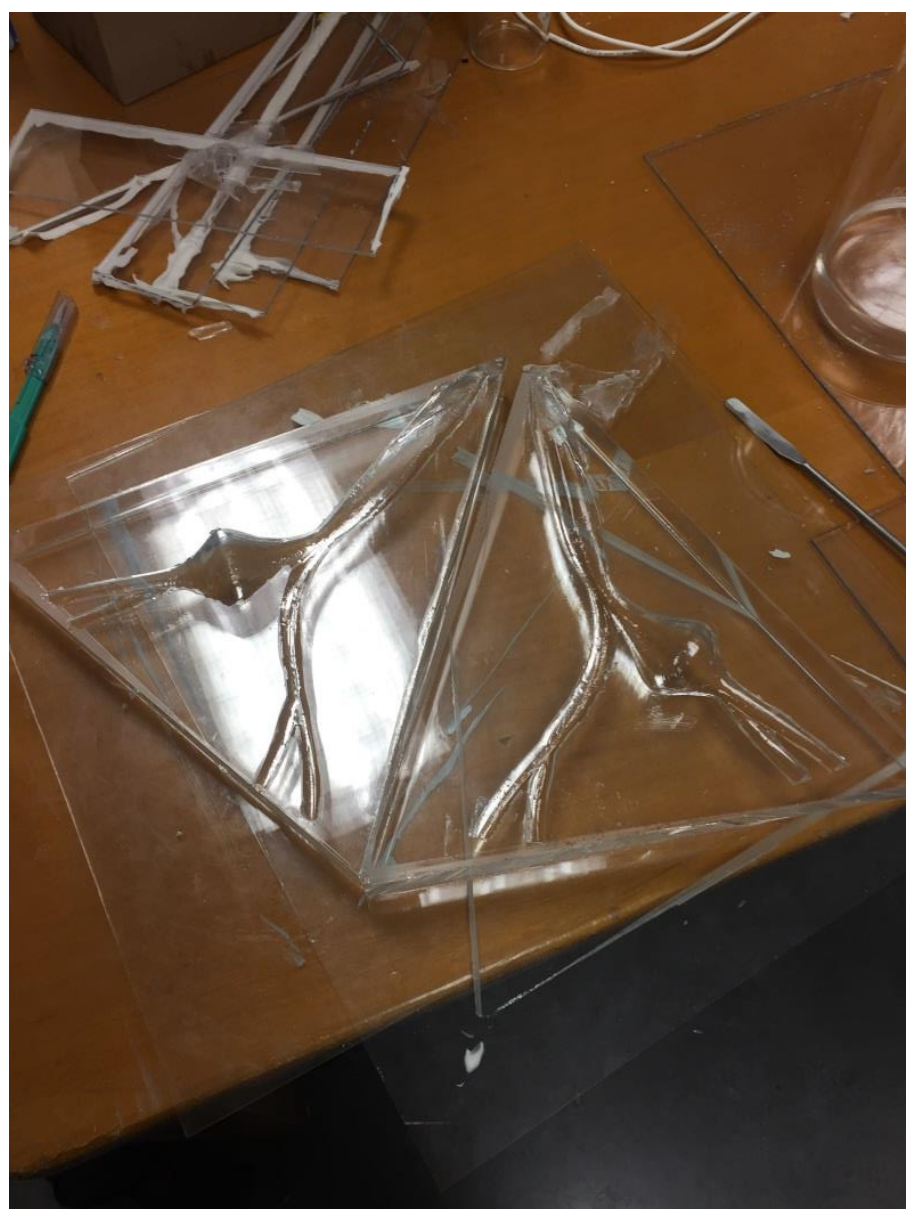

Figure 2.9: PDMS molds with 3D printed part removed

\subsubsection{PDMS Bonding}

It was decided by the author to forgo plasma bonding for reasons stated in section 2.1.3, and bond the two half constructs to each other with a thin layer of PDMS. After the half constructs were cleaned with IPA, a thin layer of PDMS was painted on to the insides of the half vessels. There was caution taken to get the PDMS as close to the vessel walls as possible to ensure a tight seal while keeping enough distance so that the excess PDMS would not drip into the vessel. This technique proved to be more challenging than expected.

The two pieces were joined together in mirror formation. There immediately appeared to be excess PDMS dripping into the construct which could alter the fluid 
dynamics of the vessel. The two pieces had to be pulled apart and the excess PDMS was removed. Unfortunately the process had to be repeated several times until, by visual inspection, there was no more PDMS leaking into the vessel. One other major complication that arose from the PDMS bonding was drift. After the two halves were joined, the two pieces began to drift which could ultimately lead to misalignment of the vessels. This would be considered a fatal error in terms of the study so it was important that this problem was properly addressed. The first line of action was to apply excessive uniform weights to the joined half vessels. Although this solution initially mitigated the problem, eventually the two pieces continued to drift. The ultimate solution to the issue was applying multiple pins that penetrated both layers of the construct but did not disrupt the vessel (figure 2.10).

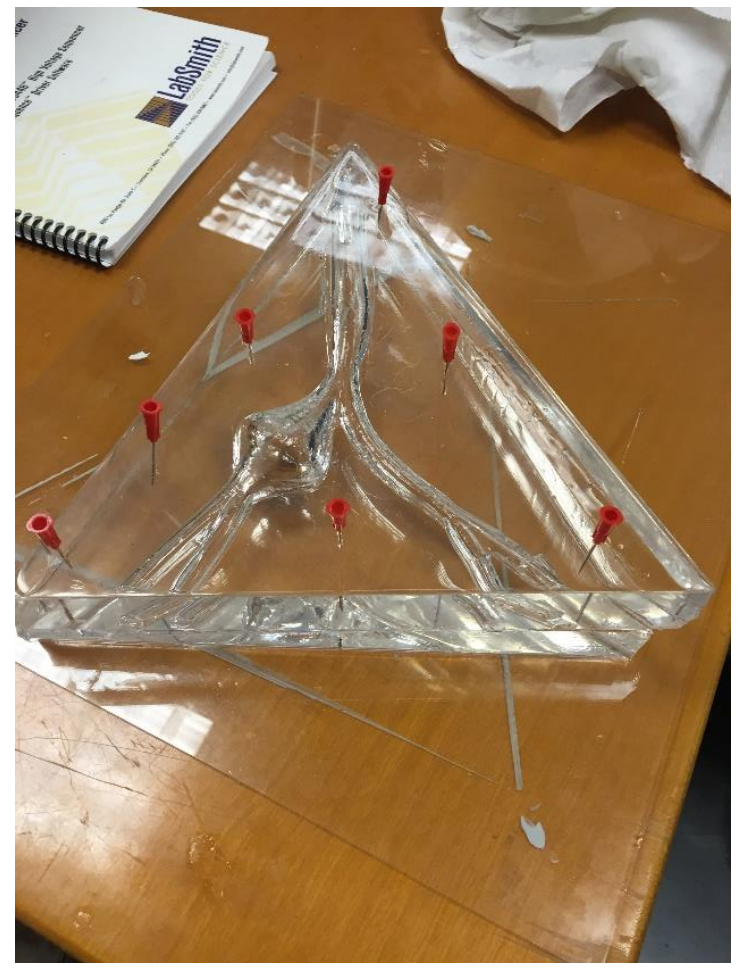

Figure 2.10: Pins inserted into the construct to inhibit drift 
The only limitation to this method was that uniform weights could not be applied to the construct which would result in some air bubbles throughout the construct. The final construct was cleaned using IPA.

\subsection{Imaging}

\subsubsection{Recording}

The recording process was completed using the camera from an iPhone 6 . In order to stabilize the camera at a sufficient focal distance, a table structure was built using a cardboard box as the table top surface. The phone was placed on top of the box and anchored down with packing tape. The model was placed vertically across from the table on top of four risers to allow for proper outflow. The tubing that was connected to the aneurysm cavity was attached to a pump that had an output rating of $2.5 \mathrm{~L} / \mathrm{min}$ which is within the spectrum of the output volumetric flow rates of a human aorta. The pump was placed in a bucket of water and water was pumped through the model and tilted to eliminate any air bubbles before recording.

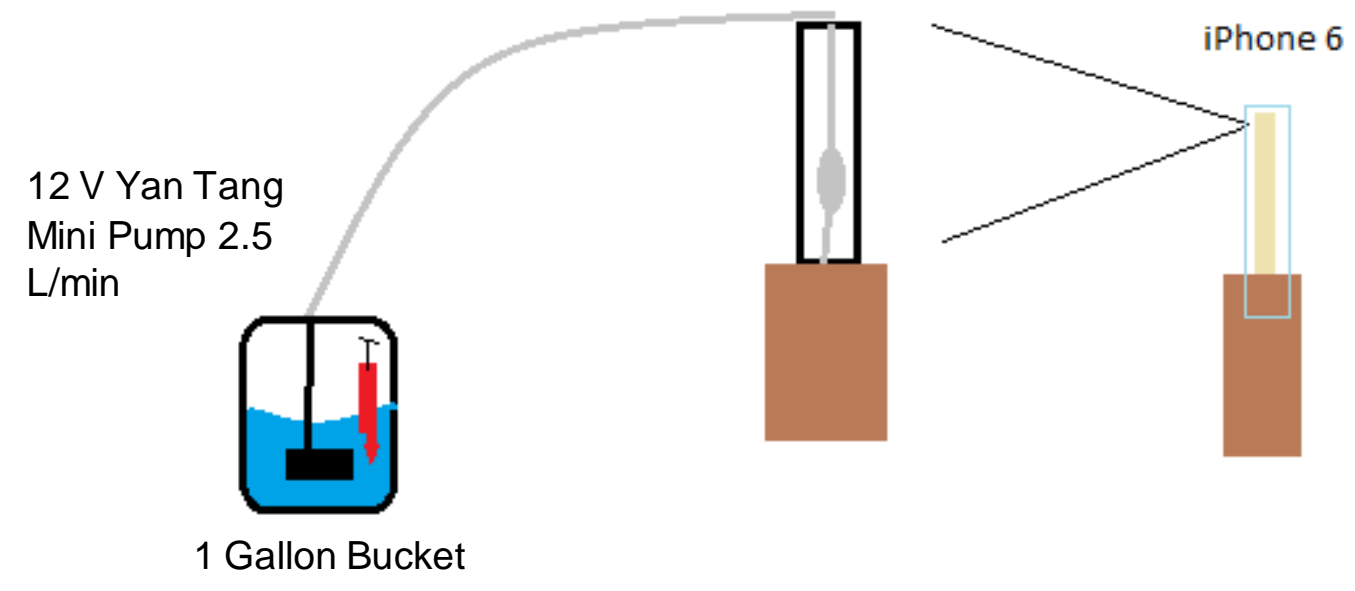

Figure 2.11: Recording Process Cartoon 


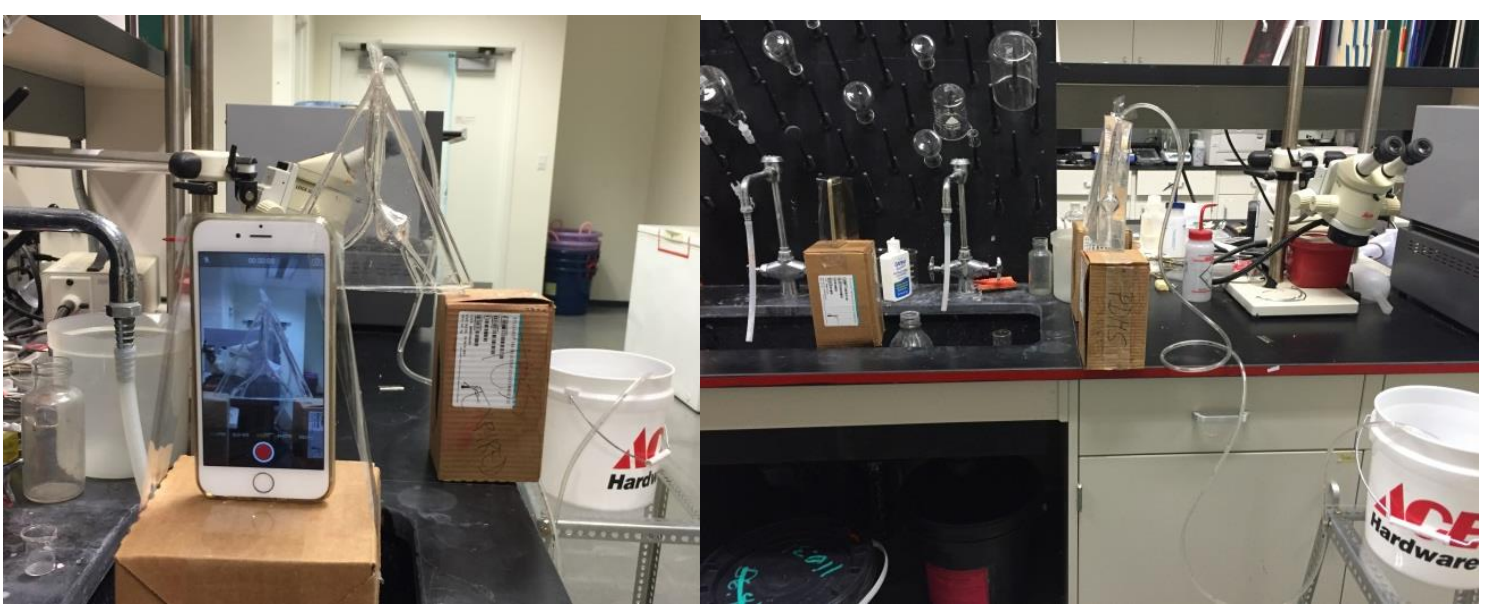

Figure 2.12: Actual photos of the imaging process

Due to the small inlet and dramatic increase in diameter, the inlet was not reaching a uniform velocity profile. This issue had to be mitigated by plugging the two nonaneurysmal branches to encourage flow through the branch of interest. The reduction of volume by under half was allowed based on the assumption that the heart can pump blood at about $5 \mathrm{~L} / \mathrm{min}$, essentially twice the flow rate of the pump. While the pump remained running, the camera began recording and red dye was injected into the inlet of the pump.

\subsubsection{Image Processing}

The individual frames of the video recordings were then compiled into a series of still images, each frame representing 0.02 seconds. Those images were then opened in the ImageJ software, and a threshold was set in RGB mode. This allowed for a more consistent reading by providing hard lines where the dye was as opposed to a gradient which was an artifact of mixing clear water with the food dye. 


\subsubsection{Image Analysis}

The images that were gathered and had thresholds added to the m were then analyzed to extract velocity values. The furthest downstream threshold pixel value was compared to furthest downstream pixel value on the proceeding frame. This procedure was developed by the author along with Daniel Greinke. These values were calculated as distances in units of pixels and then converted to units of meters. Using the distances and times between frames, velocities were calculated between pixel coordinates. These pixel coordinates were then transformed into COMSOL MULTIPHYSICS $₫$ coordinates using the coordinate transformation (Eq. 2.1 and Eq. 2.2) where $\alpha$ is the angle of rotation and $\left(x_{0} ; y_{0}\right)$ is the origin) to adjust for rotation angle and origin displacement.

The common feature of the bifurcation was used as the origin to relate the two images.

$$
\begin{aligned}
& x=x^{\prime} \cos \alpha-y^{\prime} \sin \alpha+x_{o} \\
& y=x^{\prime} \sin \alpha-y^{\prime} \cos \alpha+y_{o}
\end{aligned}
$$

\subsection{COMSOL MULTIPHYSICS ® Validation}

Once the geometry of the half vessel was transferred into the COMSOL MULTIPHYSICS $®$ software, multiple studies were run to validate the COMSOL MULTIPHYSICS $\AA$ model and to track any differences between healthy vessel and an aneurysmal vessel. For studies validating the COMSOL MULTIPHYSICS $®$ model with the empirical model, fluid properties for water were set in COMSOL MULTIPHYSICS ${ }^{\circledR}$ with a Newtonian viscosity of $0.89 \mathrm{cP}$ and a density of $1000 \mathrm{~kg} / \mathrm{m}^{3}$. For studies comparing the aneurysmal vessel to the non-aneurysmal vessel fluid properties of blood were set with a density of $1025 \mathrm{~kg} / \mathrm{m}^{3}$ with a viscosity calculated in COMSOL MULTIPHYSICS ${ }^{\circledR}$ using a Non-Newtonian Carreau Model (figure 2.10). The NonNewtonian Carreau can be explained by equation 2 where the viscosity at high shear 
rate $\eta \infty$ equals the value for the Newtonian model (i.e. $0.0035 \mathrm{~Pa}$ s) while the value at zero shear is $\eta_{0}=0.056$ Pa s. Also: $\lambda=3.313$ s and $n=0.3568$.

$$
\eta=\eta_{\infty}+\left(\eta_{0}-\eta_{\infty}\right)\left[1+(\lambda \dot{\gamma})^{2}\right]^{\frac{n-1}{2}}
$$

At high shear rates, the viscosity of the fluid converges to that of a Newtonian fluid, at low shear rates the viscosity appears to follow the power law where shear rate is a function of the partial differential of velocity.

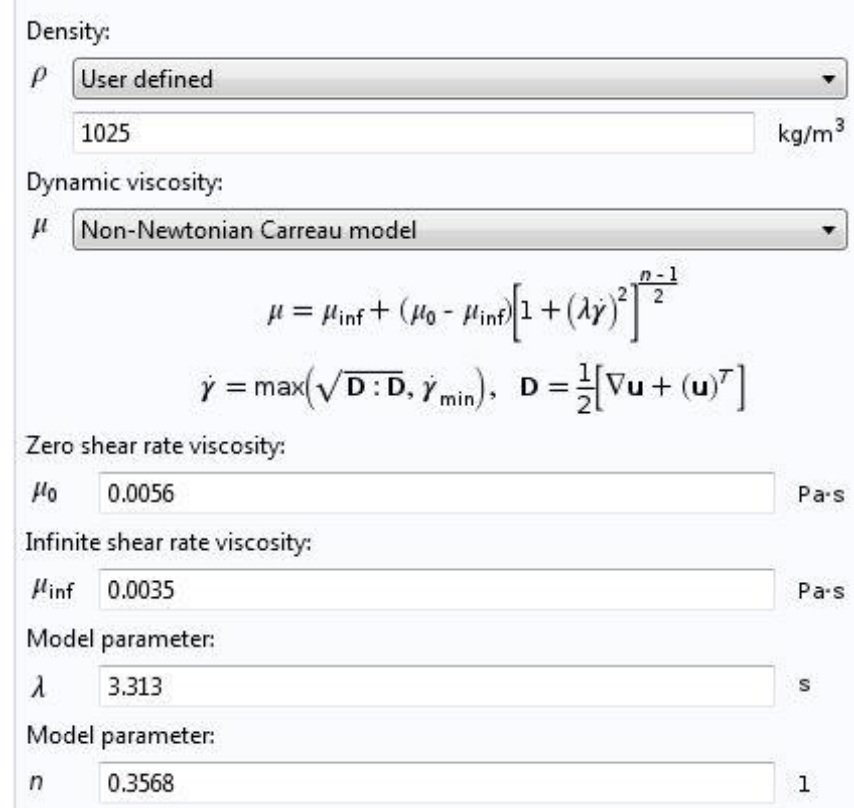

Figure 2.13: Fluid properties for the Carreau model in COMSOL MULTIPHYSICS ${ }^{\circledR}$

Laminar flow was selected for all studies based on the Reynold's number of 1905 which would indicate a laminar flow (Table 2.4). Laminar flow was selected because it accounts for both viscous and inertial effects.

$$
\begin{gathered}
R e=\frac{\text { Inertial Forces }}{\text { Viscous Force }}=\frac{\rho v D}{\mu} \\
\rho\left(\frac{d \underline{V}}{d t}+\underline{v} \cdot \underline{\nabla v}\right)=\underline{\nabla} \cdot \underline{T}+\rho \underline{g}
\end{gathered}
$$


Where $\mu$ is the viscosity ( $1 \mathrm{cP}$ for the purposes of this calculation), $\rho$ is the density (1000 $\mathrm{kg} / \mathrm{m}^{3}$ ), $\mathrm{D}$ is the diameter of the vessel, and $\mathrm{v}$ is the maximum velocity. A tetrahedral mesh calibrated for general physics was used for all models based on the number of elements created in table 2.3 (Figure 2.14).

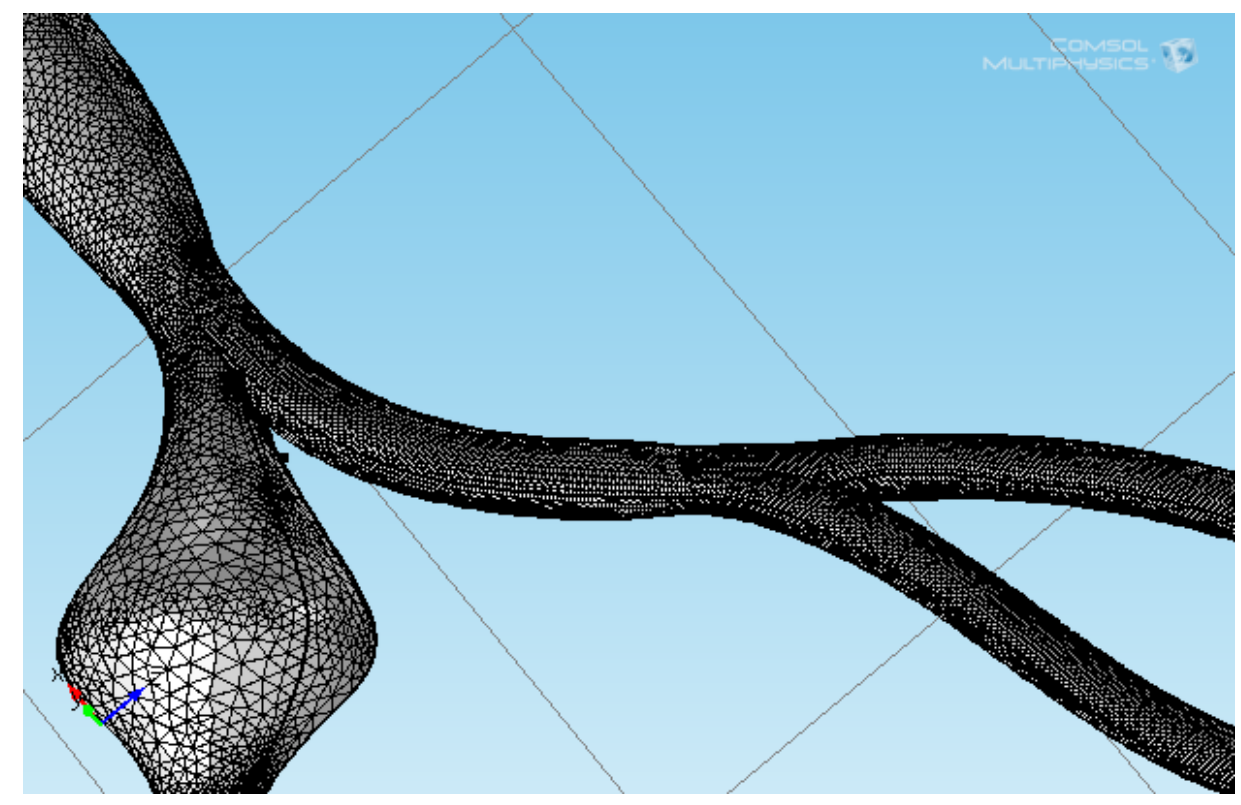

Figure 2.14: Extremely Fine Mesh in General Physics

COMSOL MULTIPHYSICS ® provides statistics regarding the amount of elements created for each mesh size. These results can be seen in Table 2.3. The amount of elements created can greatly affect the accuracy of the results of the study. The more elements created, the closer the model will be to converging to a smooth 3D surface. The following table denotes the percent difference of a measured value in relationship to the mesh setting. The desired percent difference was less than $10 \%$. The values calculated were velocity values in $\mathrm{m} / \mathrm{s}$ derived from point evaluations at the inlet of the aneurysms. 
Table 2.2 Mesh Analysis

\begin{tabular}{|c|c|c|}
\hline \hline Mesh Setting & Measured Value & 356 \\
\hline \hline Normal & 0.31782 & 255 \\
\hline Fine & 0.24665 & 53.4 \\
\hline Finer & 0.10660 & 14.3 \\
\hline Extra Fine & 0.07945 & 0 \\
\hline
\end{tabular}

From the mesh analysis, an extremely fine mesh was chosen for the study.

Table 2.3 Number of elements created based on mesh settings

\begin{tabular}{|c|c|c|c|c|}
\hline Mesh Setting & $\begin{array}{l}\text { Tetrahedral } \\
\text { Elements }\end{array}$ & $\begin{array}{l}\text { Triangular } \\
\text { Elements }\end{array}$ & Edge Elements & $\begin{array}{l}\text { Vertex } \\
\text { Elements }\end{array}$ \\
\hline Tormal & $\overline{44,429}$ & 2,722 & 589 & 59 \\
\hline Fine & 12,027 & 5,810 & 902 & 59 \\
\hline Finer & 44,642 & 14,596 & 1,513 & 59 \\
\hline Extra Fine & 120,077 & 27,210 & 2,209 & 59 \\
\hline Extremely Fine & 400,521 & 62,684 & 3,650 & 59 \\
\hline
\end{tabular}


Table 2.4 Flow Regime According to Reynold's Number

\begin{tabular}{|c|c|c|}
\hline \hline Re<<1 & Re<2100 & Re $>4000$ \\
\hline Stoke's Flow[24] & Laminar Flow [24] & Turbulent Flow [24] \\
\hline \hline
\end{tabular}

The initial value at the inlet was set at $0.2397 \mathrm{~m} / \mathrm{s}$. This was calculated based on the measured volumetric flow rate exerted by the pump, assuming uniform flow field across the inlet. The boundary conditions at all of the outlets were set at a pressure of 0 . In order to limit the computing time, a half vessel was used with a symmetry condition on the flat plane of the vessel. After all of the properties had been set up and the mesh was built, the studies were run. The first studies that were run on both the non-aneurysmal vessel and the aneurysmal vessel were stationary studies to retrieve both qualitative surface plots of the velocity and shear stress as well as quantitative line grafts and point evaluations of the shear stresses along the inner and outer walls of the vessel. Line graphs and point evaluations were also computed to evaluate the velocity and shear stress along the horizontal center line of the aneurysm. Additional qualita tive results from the stationary study were created, such as stream line depiction of the velocity throughout the vessel and arrow plots indicating the direction and magnitude of the velocity within the aneurysm. A second study was computed using a time-dependent model. The objective of this study was to create a movie of the velocity propagation throughout the aneurysmal vessel and non-aneurysmal vessel. The study computed data points every 0.1 second for a total of 1 second. No quantitative data was extracted from this study.

The final COMSOL MULTIPHYSICS $®$ study that was performed was a time dependent steady state particle study. A particle tracking model was implemented after the stationary, steady state laminar model had been computed. COMSOL 
MULTIPHYSICS ${ }^{\circledR}$ uses the solutions from the laminar study and applies a particle release function. The fluid properties that were assigned for the laminar model remain the same for the particle tracking model with the addition of a drag force. The drag force that is applied to each model is represented by the following equations where $\mathrm{pp}$ is the density of the particle $\left(1060 \mathrm{~kg} / \mathrm{m}^{3}\right), d_{p}$ is the diameter of the particle $(80 \mu \mathrm{m}), m_{p}$ is the mass of the particle (542 ng), $\mu$ is the viscosity of the fluid (taken from laminar simulation), and $\mathrm{v}$ is the particle velocity (taken from laminar study). 125 particles were released from the inlet every 0.01 seconds. The boundary conditions at the walls were set so that the particles would bounce off the walls and disappear at the outlet.

$$
\begin{gathered}
F=\frac{1}{\tau_{p}} m_{p}(u-v) \\
\tau_{p}=\frac{\rho_{p} d_{p}}{18 \mu}
\end{gathered}
$$




\section{RESULTS}

\subsection{Experimental Findings}

The experimental portion of the study yielded both quantitative and qualitative findings. The quantitative results provided a velocity value through the aneurysm which can be found in figure 3.23. The qualitative results provided the findings of a vortex that was developed in the aneurysm during the experiment (Figure 3.1).

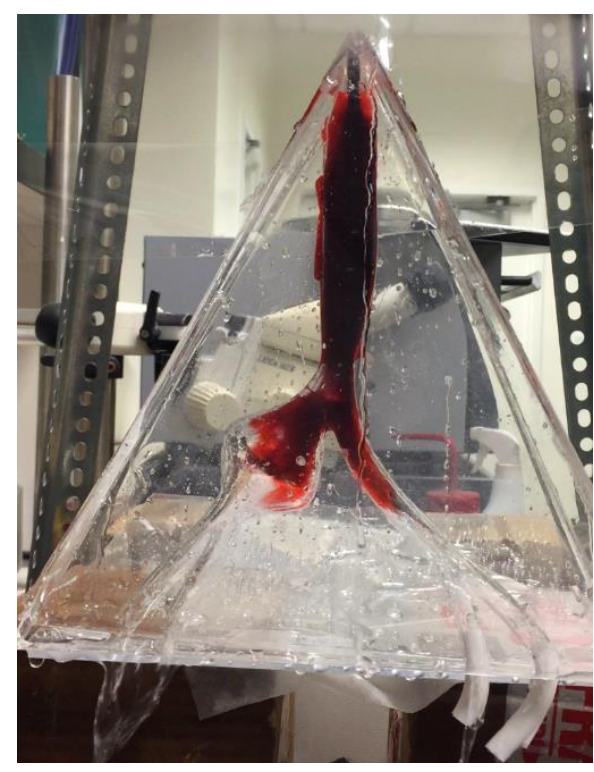

Figure 3.1: Still Image Capturing the Vortex Effect in the Aneurysm at 11 seconds

There also appeared to be very minimal lateral leakage which would indicate that the PDMS bonding was successful.

\subsubsection{Statistical Analysis}

The statistical analysis for the experimental trials against the COMSOL MULTIPHYSICS ( ) trials was calculated by using 2-Sample T-Tests and Paired 2-Sample T-Tests in Minitab. The data from both the paired and non-paired tests suggest that there is not enough statistical evidence to conclude a difference in the velocities gathered from 
empirical model and the COMSOL MULTIPHYSICS ${ }^{\circledR}$ model. For this study, alpha was set at 0.05 , the confidence interval was set at $95 \%$ and the alternative hypothesis describes a significant difference in samples.

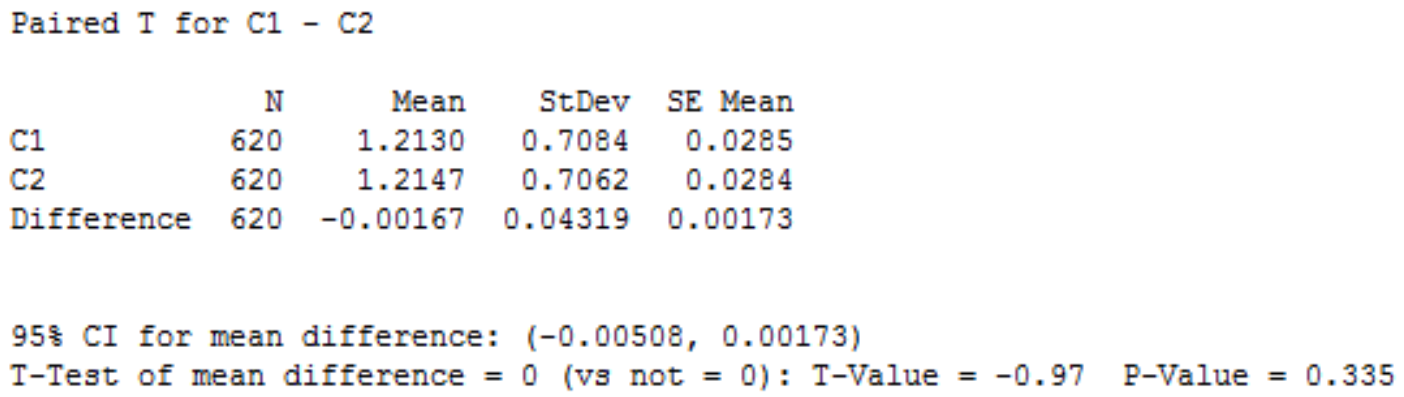

Figure 3.2: Illustration of how the statistics were derived

\subsection{In Silico Findings}

\subsubsection{Cylinder Validation}

A simple cylinder was used to validate the accuracy of their COMSOL MULTIPHYSICS (B parameters. In this verification, the cylinder was used to prove COMSOL MULTIPHYSICS ${ }^{\circledR}$ 's fluid physics to analytical equations. The cylinder was $5 \mathrm{~mm}$ in diameter and $10 \mathrm{~cm}$ long. An average velocity of $0.3 \mathrm{~m} / \mathrm{s}$ and a pressure drop of $115 \mathrm{~Pa}$ were used as boundary conditions. A stationary study was run using the same mesh that was used for the CIA simulation. The equations that were used to verify the COMSOL MULTIPHYSICS $®$ simulation were Hagen-Pouseuille and the wall shear stress on for a straight tube. The average velocity and average wall shear stress were then simulated for blood as a Newtonian fluid in the cylinder. The results can be seen in Table 3.1 


$$
\begin{gathered}
\text { Vave }=\frac{R^{2}\left(P_{0}-P_{L}\right)}{8 \mu L} \\
\tau_{W}=\frac{\left(P_{0}-P_{1}\right) R}{2 L}
\end{gathered}
$$

Table 3.1 Verification of COMSOL MULTIPHYSICS $®$ with a cylinder using common fluid dynamic equations

\begin{tabular}{|c|c|c|}
\hline \hline & Velocity (m/s) & $\begin{array}{c}\text { Shear Stress of } \\
\text { the Wall }(\mathrm{Pa})\end{array}$ \\
\hline & Newtonian & Newtonian \\
\hline Calculation & 0.3 & 1.44 \\
\hline Simulation & 0.30017 & 1.449 \\
\hline$\%$ Error & 0.057 & 0.6 \\
\hline \hline
\end{tabular}

COMSOL MULTIPHYSICS $®$ proved to be an accurate simulation software with very little error.

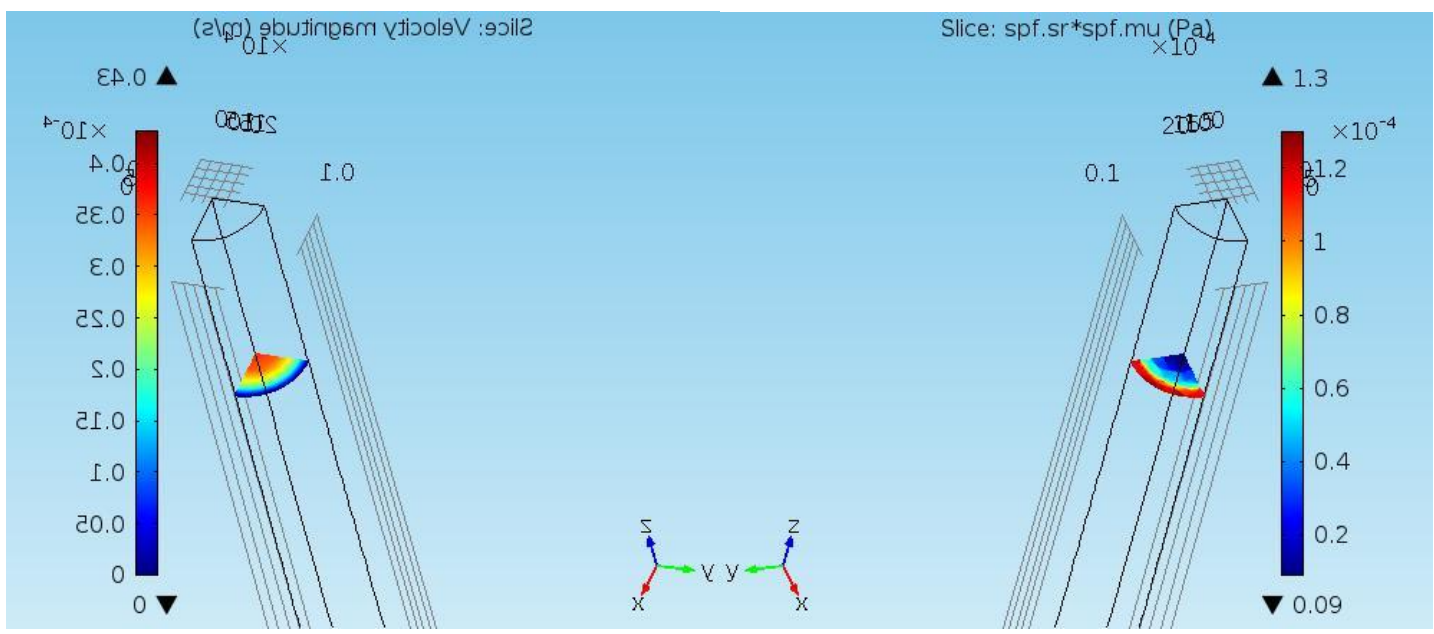

Figure 3.3: Cylinder verification 
The left image shows the resultant velocity and the right image shows the resultant shear stress.

\subsubsection{Graphic Results}

Below is a graphic depicting surface plots generated by COMSOL MULTIPHYSICS $®$ graphically depicting the intensities on a color gradient of the velocities in the aneurysmal (figure 3.4) and non-aneurysmal vessels (figure 3.5) as well as surface plots of the shear stress in the aneurysmal (figure 3.6) and non-aneurysmal vessels (figure 3.7).

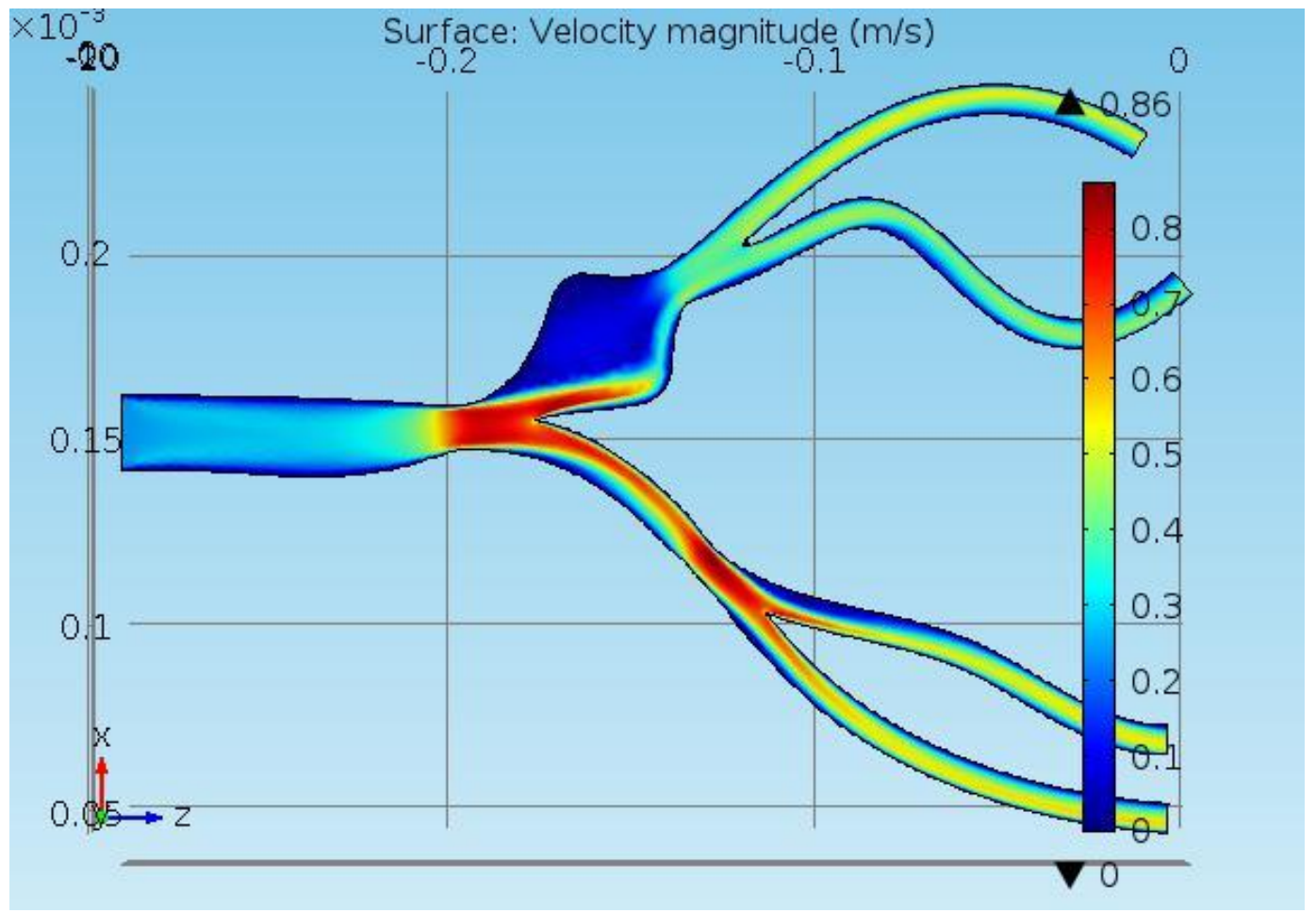

Figure 3.4: Surface plot of velocity in the aneurysmal vessel 


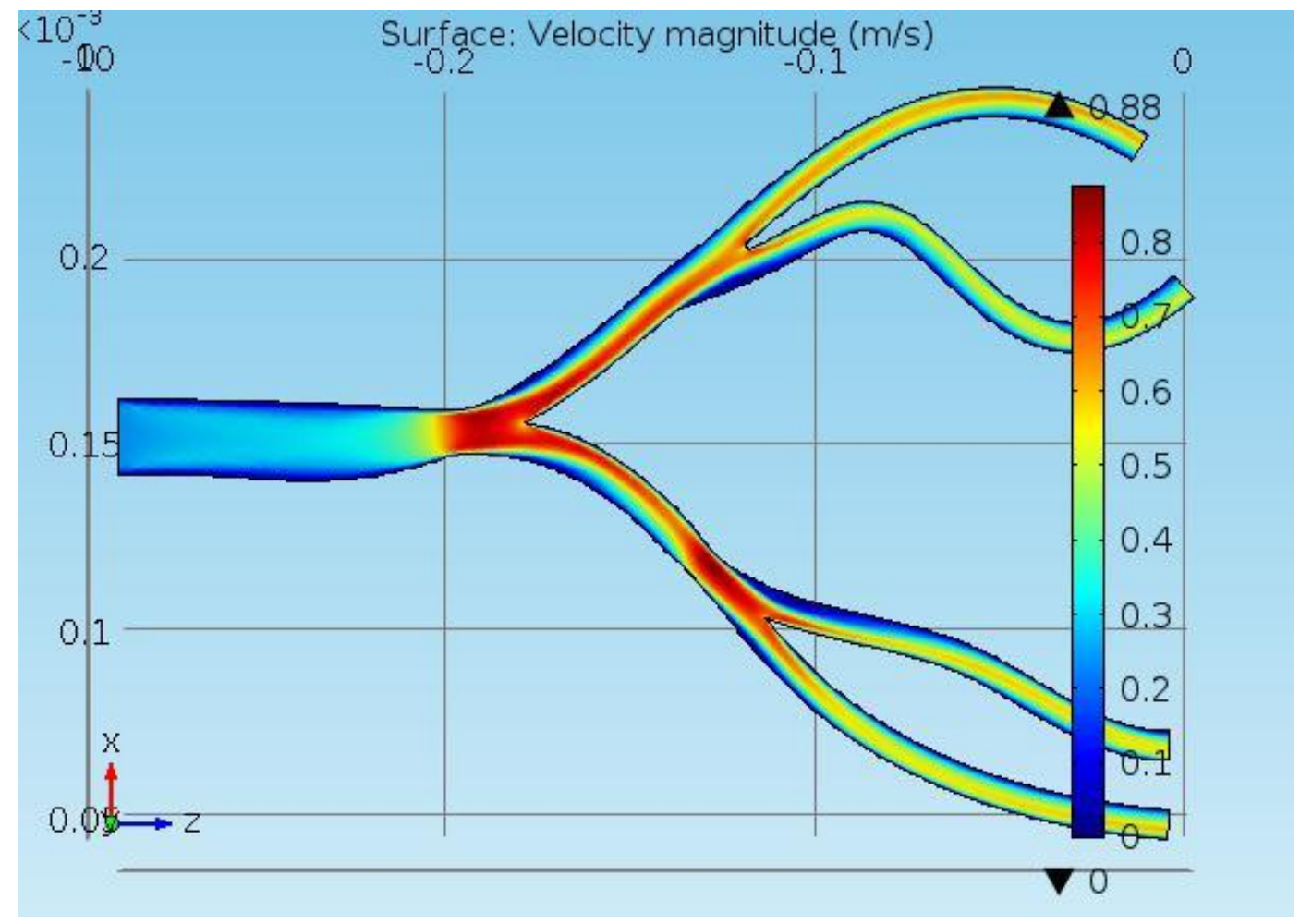

Figure 3.5: Surface plot of velocity in the non-aneurysmal vessel

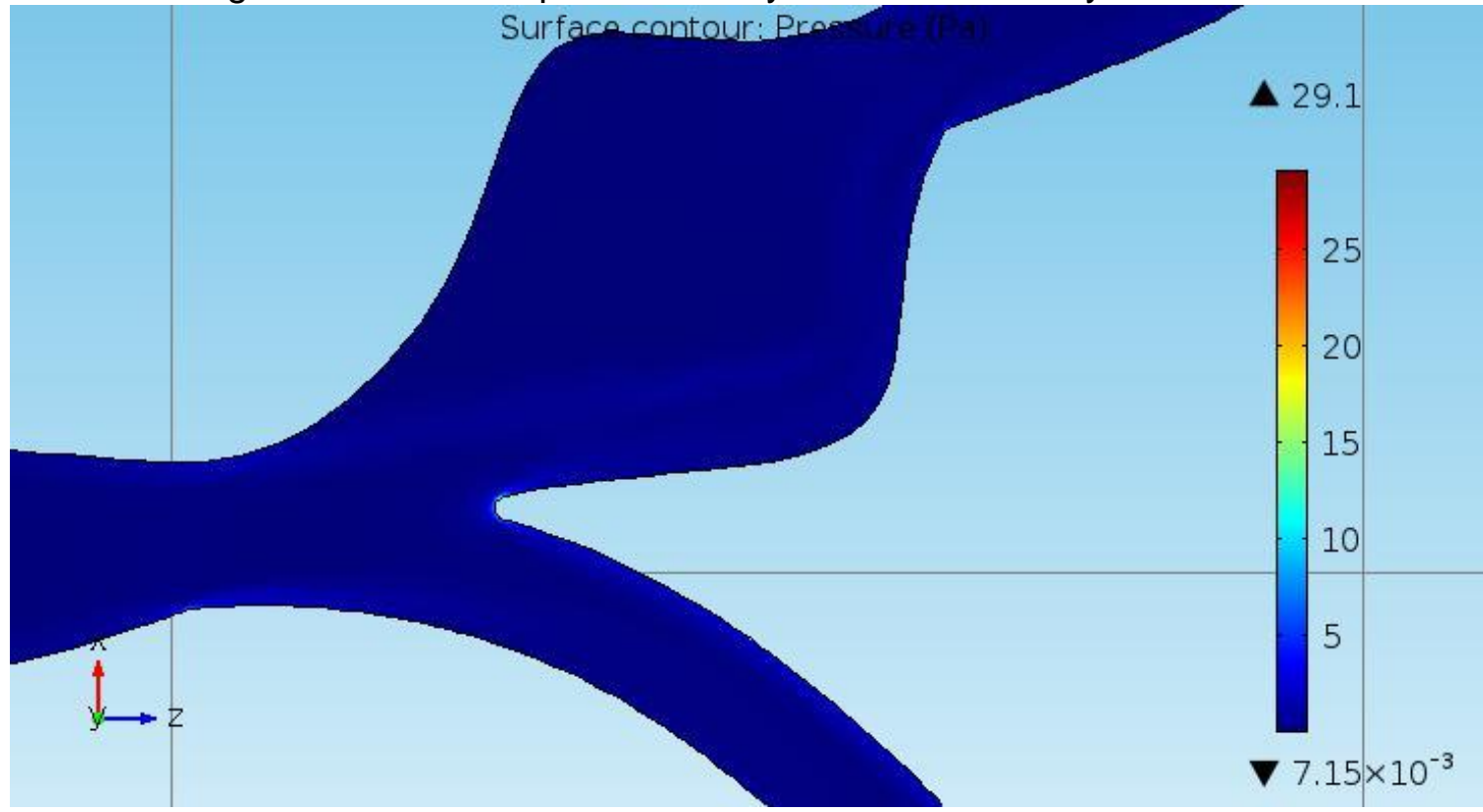

Figure 3.6: Zoomed Surface plot of shear stress in $\mathrm{Pa}$ in the aneurysmal vessel 


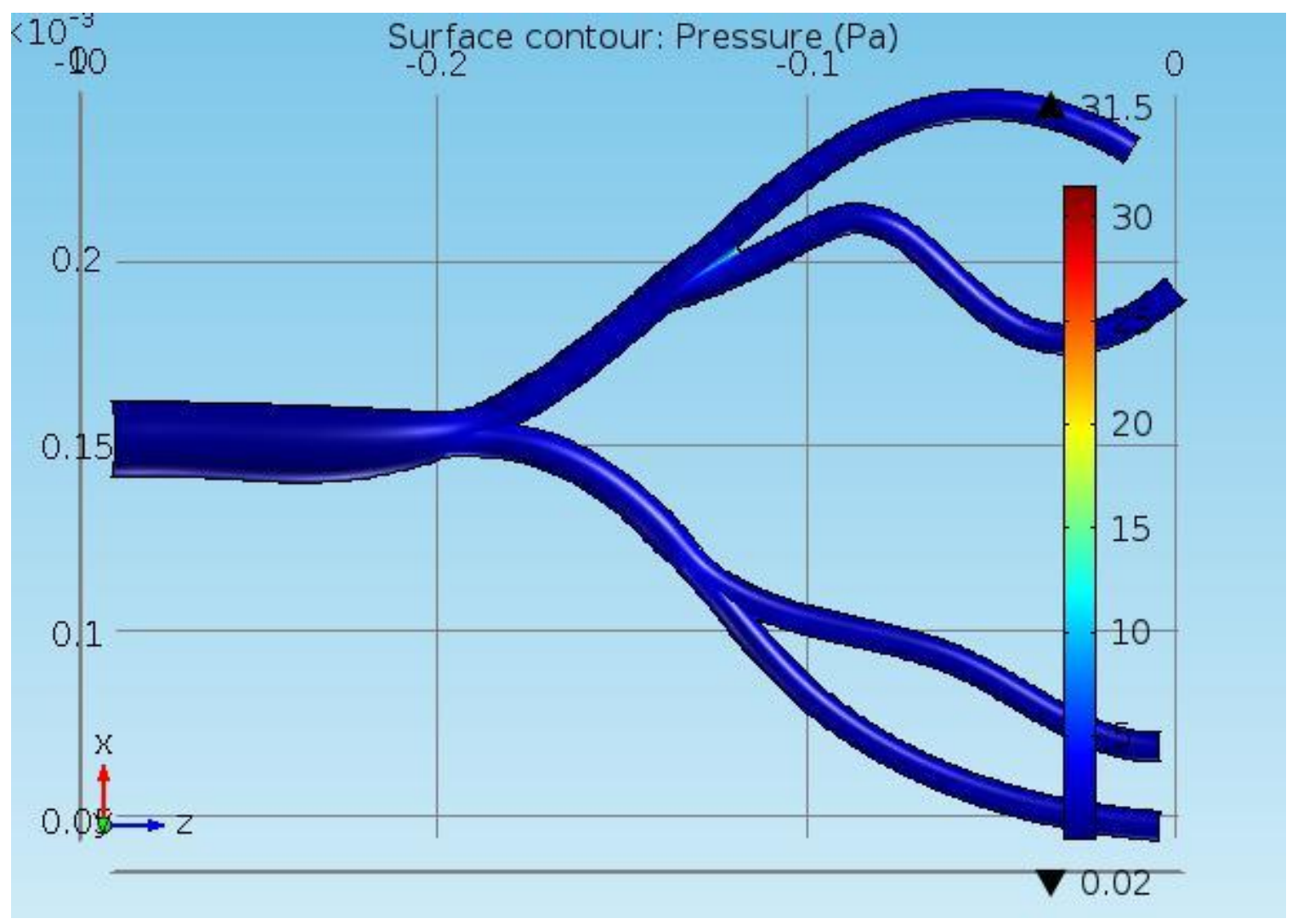

Figure 3.7: Surface plot of shear stress in $\mathrm{Pa}$ in the non-aneurysmal vessel

The interior wall sees the highest velocities while the exterior wall sees very low velocities in a circular formation. There is limited shear stress being exerted on the vessel which is why a zoomed in portion of the image is provided to show that the majority of the shear stresses occur at the common iliac bifurcation.

Additional qualitative results were calculated such as the streamline velocities in the aneurysmal (figure 3.8) and non-aneurysmal vessels (figure 3.9). 


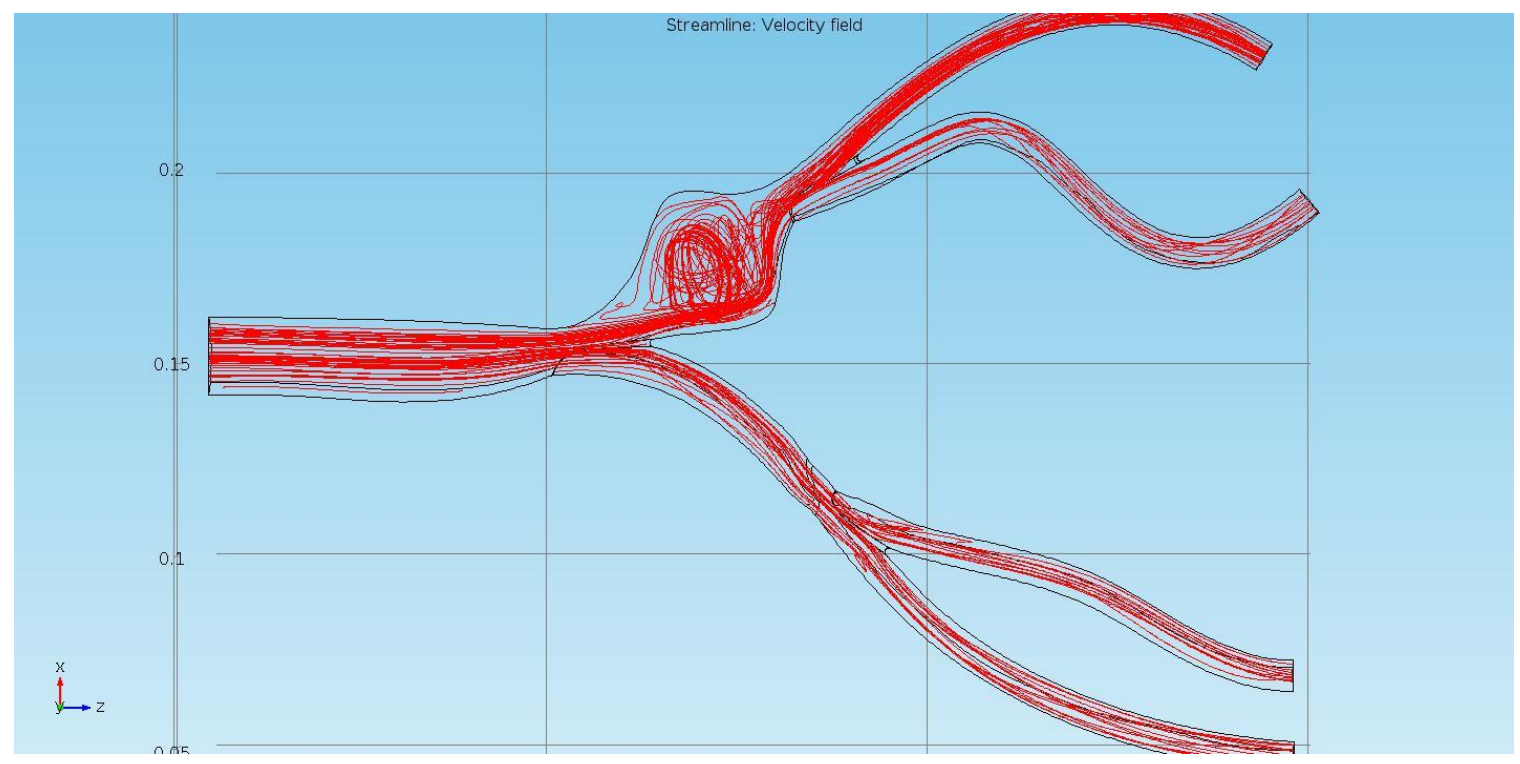

Figure 3.8: Streamline velocity of the aneurysmal vessel

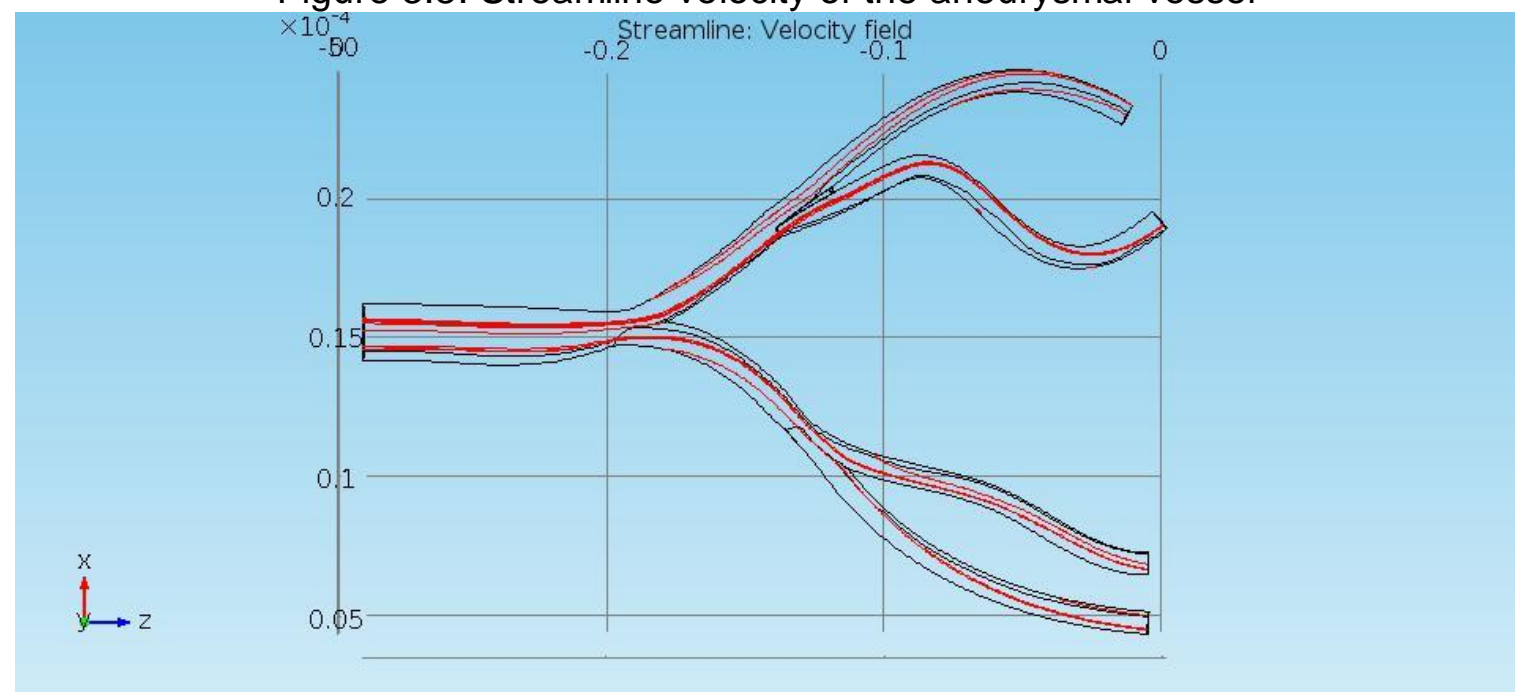

Figure 3.9: Streamline velocity in a non-aneurysmal vessel

The streamline velocity plots show the path that a given fluid particle is likely to take under the specified boundary conditions. The aneurysmal vessel shows a vortex-like structure forming in the aneurysm. It also shows a much more convoluted flow path than the non-aneurysmal vessel. The non-aneurysmal vessel shows clean, organized velocity paths, indicating that the walls in a healthy vessel would be less likely to experience pressures arising from fluid impacting the walls at a non-tangential angle. 
The final qualitative result from COMSOL MULTIPHYSICS $\AA$ shows an arrow plot of the aneurysm (figure 3.10).

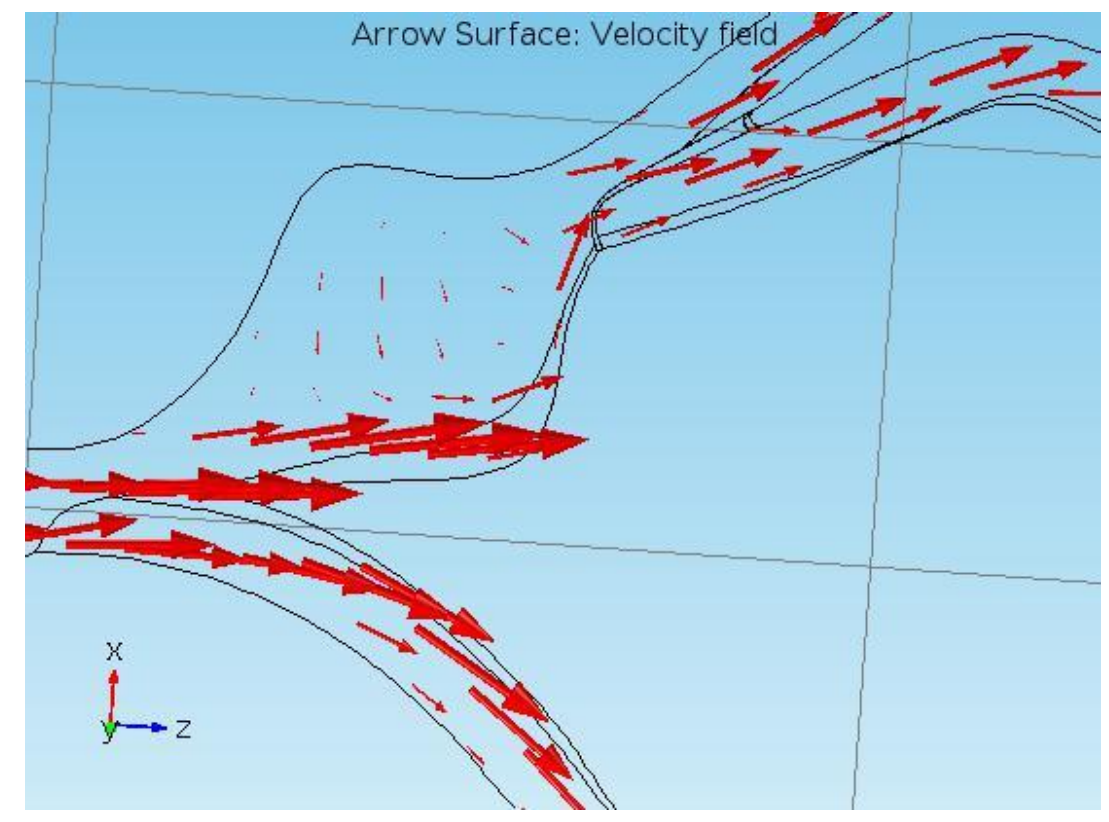

Figure 3.10: Arrow plot depicting the velocities of the fluid

This graphic explicitly shows the direction and magnitudes of the fluid flow within the aneurysm. Some important things to note are the direction of the velocities on the lateral side of the aneurysm. These arrows indicate that the fluid is actually flowing upstream causing a vortex within the aneurysm, a phenomenon shown in the empirical model. These velocities, although small, result in direct pressure from fluid flow contacting the wall orthogonally. This impact can result in the further expansion of the aneurysm.

\subsubsection{Numerical Results}

A graphical depiction of the numerical results can be summarized using line graphs. The aneurysmal and non-aneurysmal vessels line graphs were created to analyze the shear stress along the inner and outer edges of the aneurysmal branch. For the outer edge 
analysis, the only relevant information occurs along the aneurysm which correspond s to an arc length of 0.1 to 0.18 .

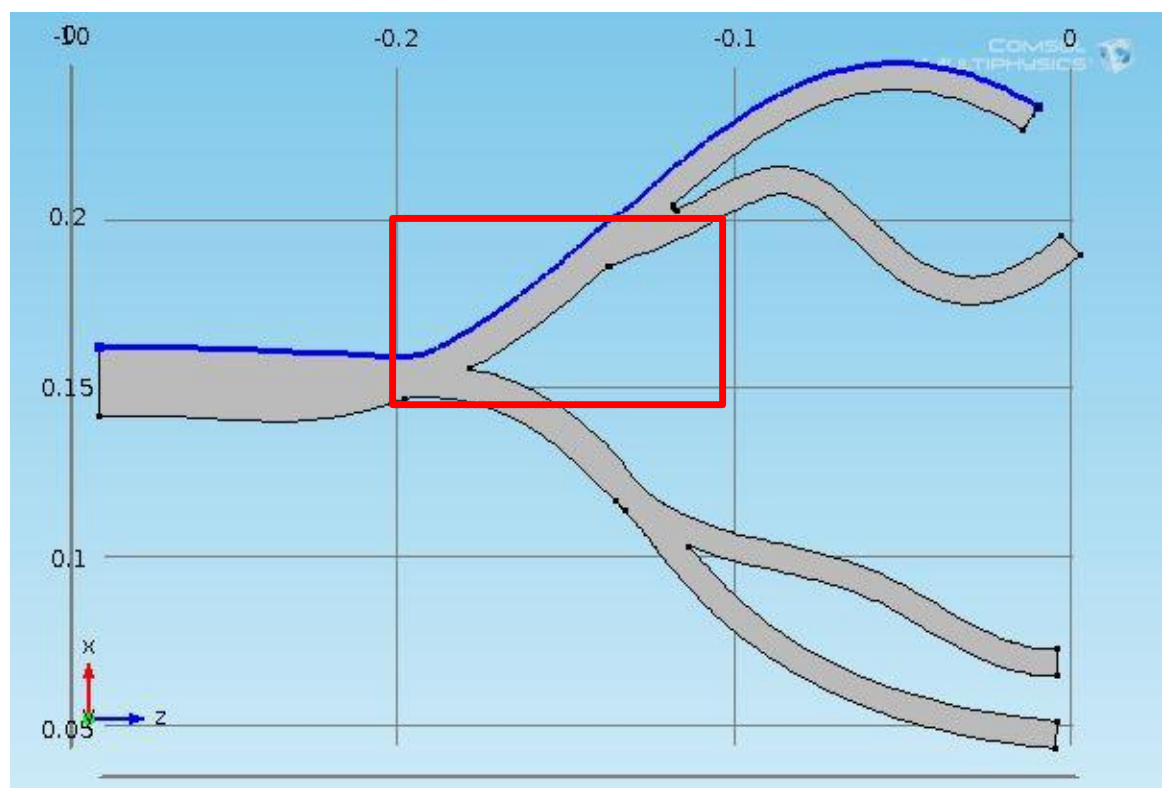

Figure 3.11: Blue line depicting the outer edge of the vessel from which the shear stress data was extracted

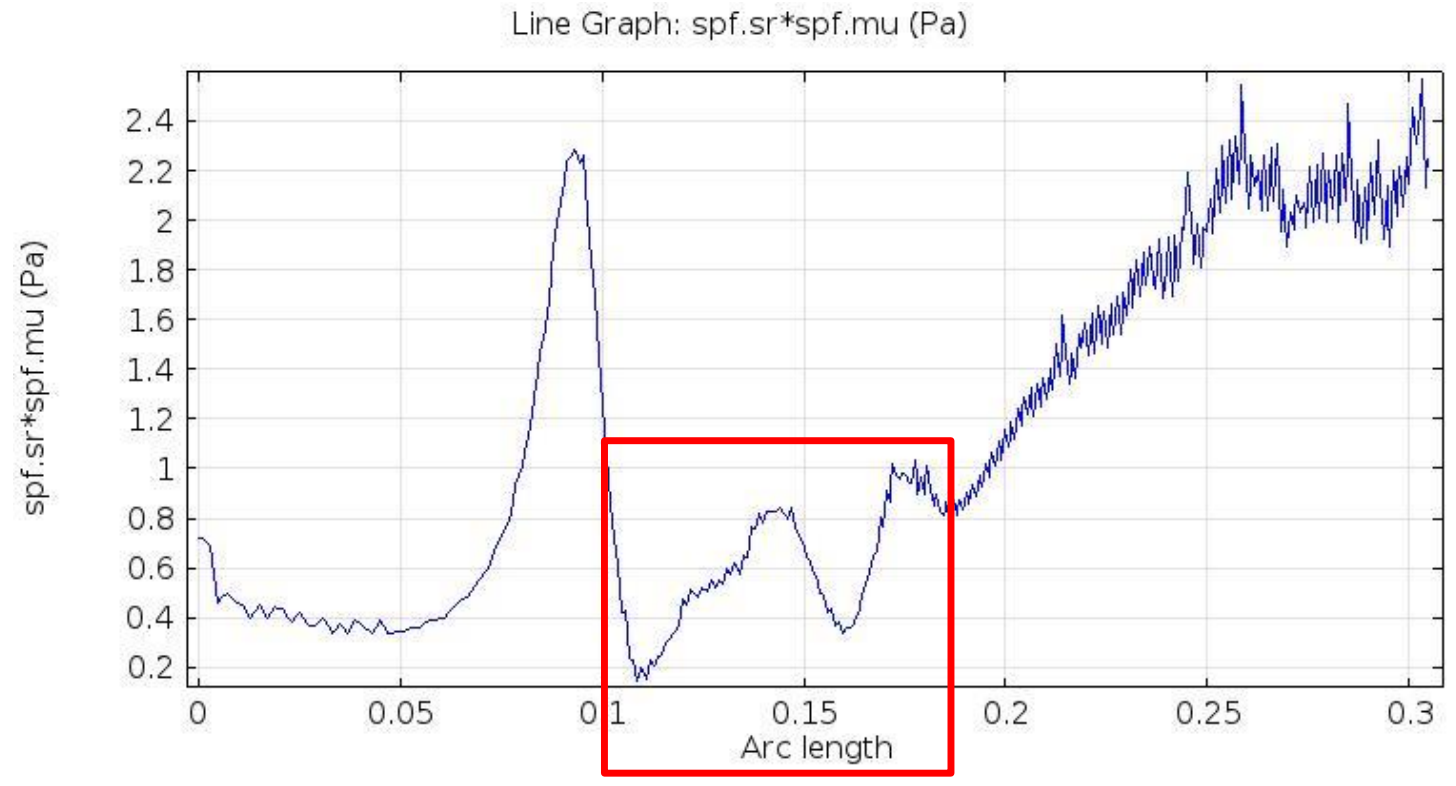

Figure 3.12: Line graph of the shear stress that occurs along the outer edge of the vessel in the non-aneurysmal vessel 


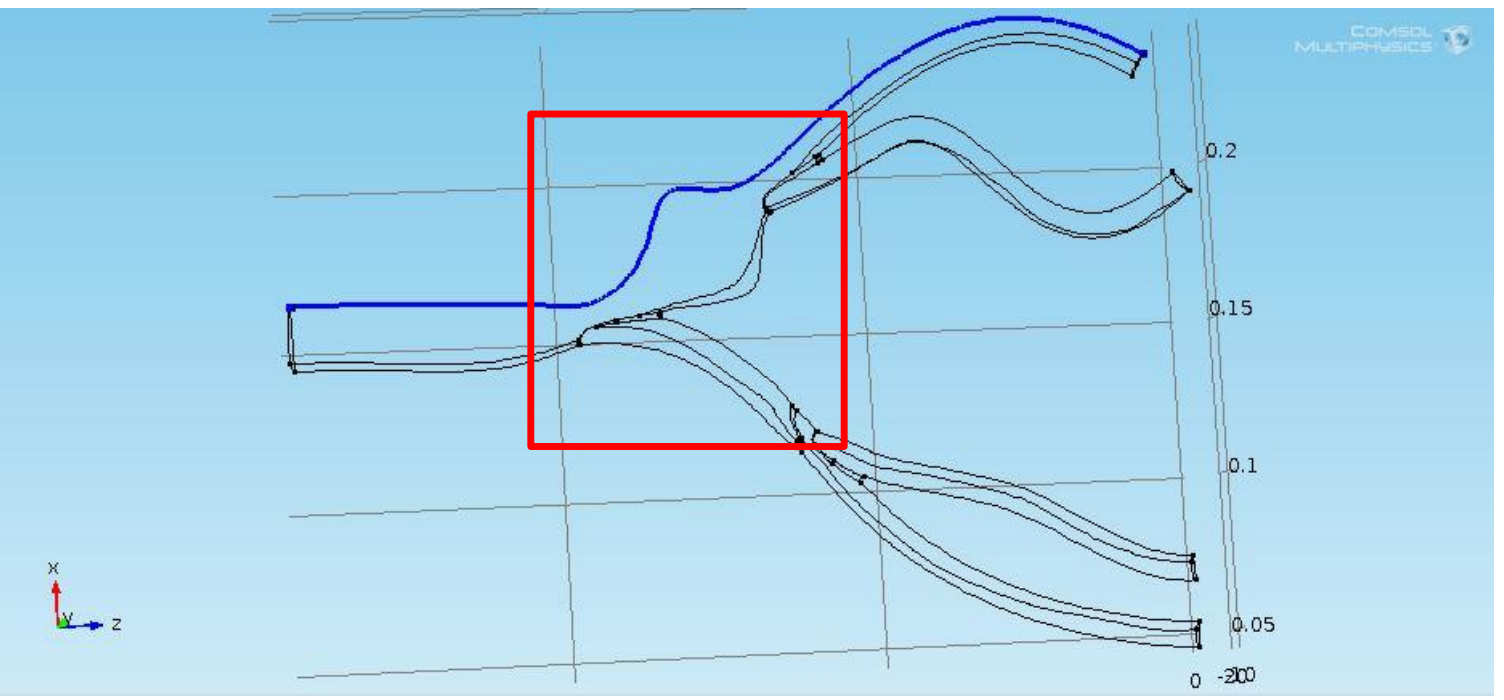

Figure 3.13: Blue line depicting the outer edge of the vessel from which the shear stress data was extracted

Line Graph: spf.mu*spf.sr (Pa)

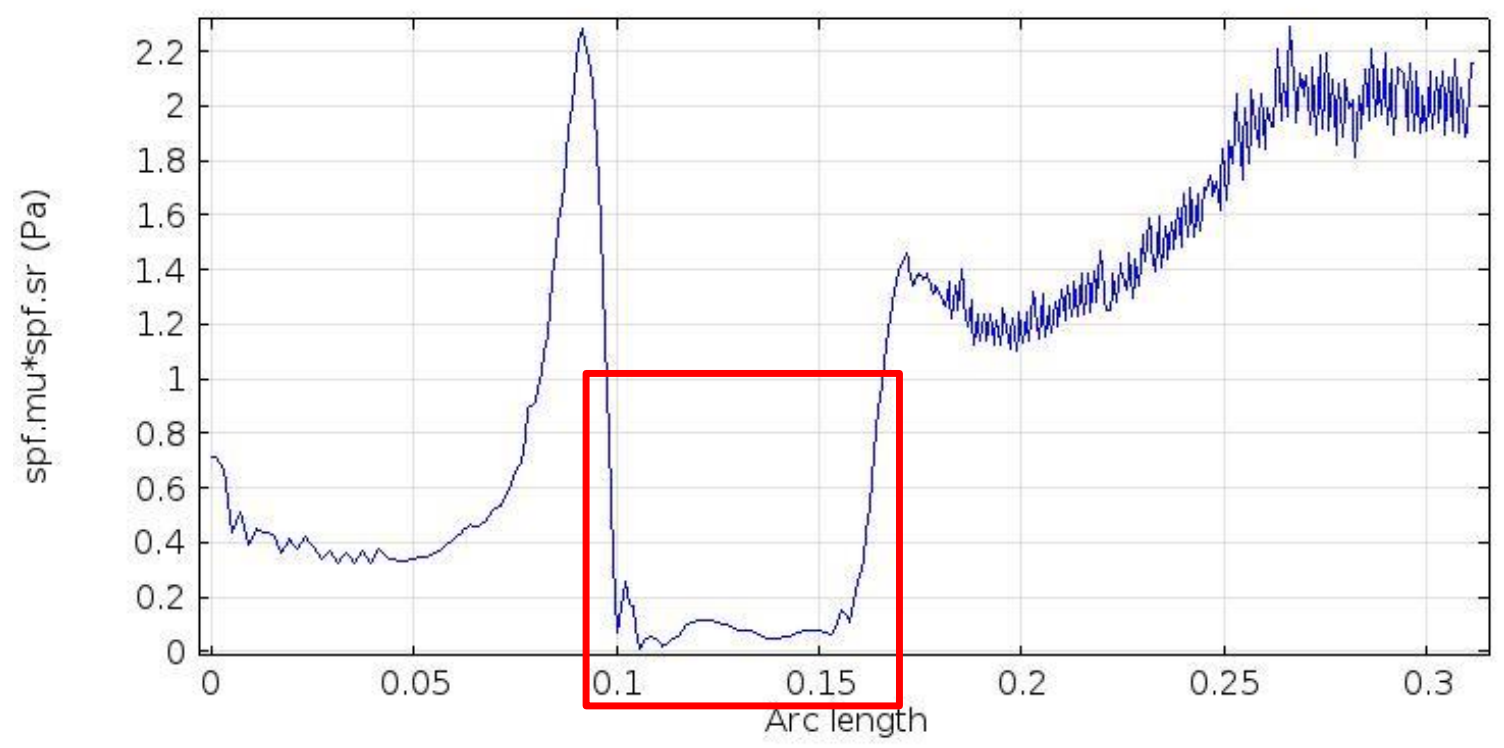

Figure 3.14: Line graph of the shear stress that occurs along the outer edge of the vessel in the aneurysmal vessel

The line graph indicates a sudden drop at the location of the aneurysm. This follows closely with the qualitative values that were derived that indicate low shear stress and low velocities in the aneurysm. The non-aneurysmal vessel experiences a more variable increase in shear stress and at a larger magnitude than the aneurysmal vessel. This could lead to thrombus formation along the outside edge of the aneurysm. 
For the inner edge data, the entire length of the arc is of relevance.

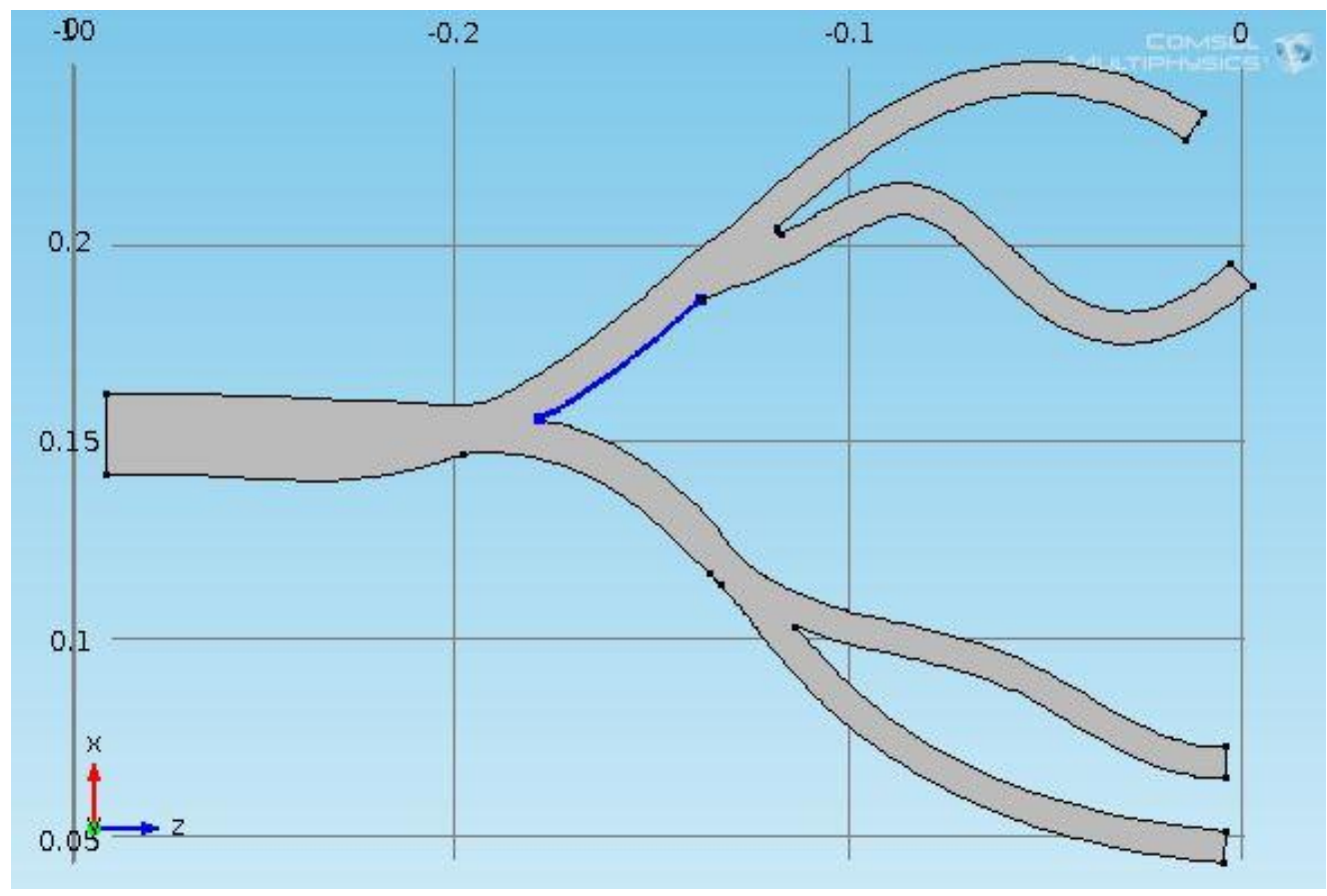

Figure 3.15: Blue line depicting the inner edge of the vessel from which the shear stress data was extracted

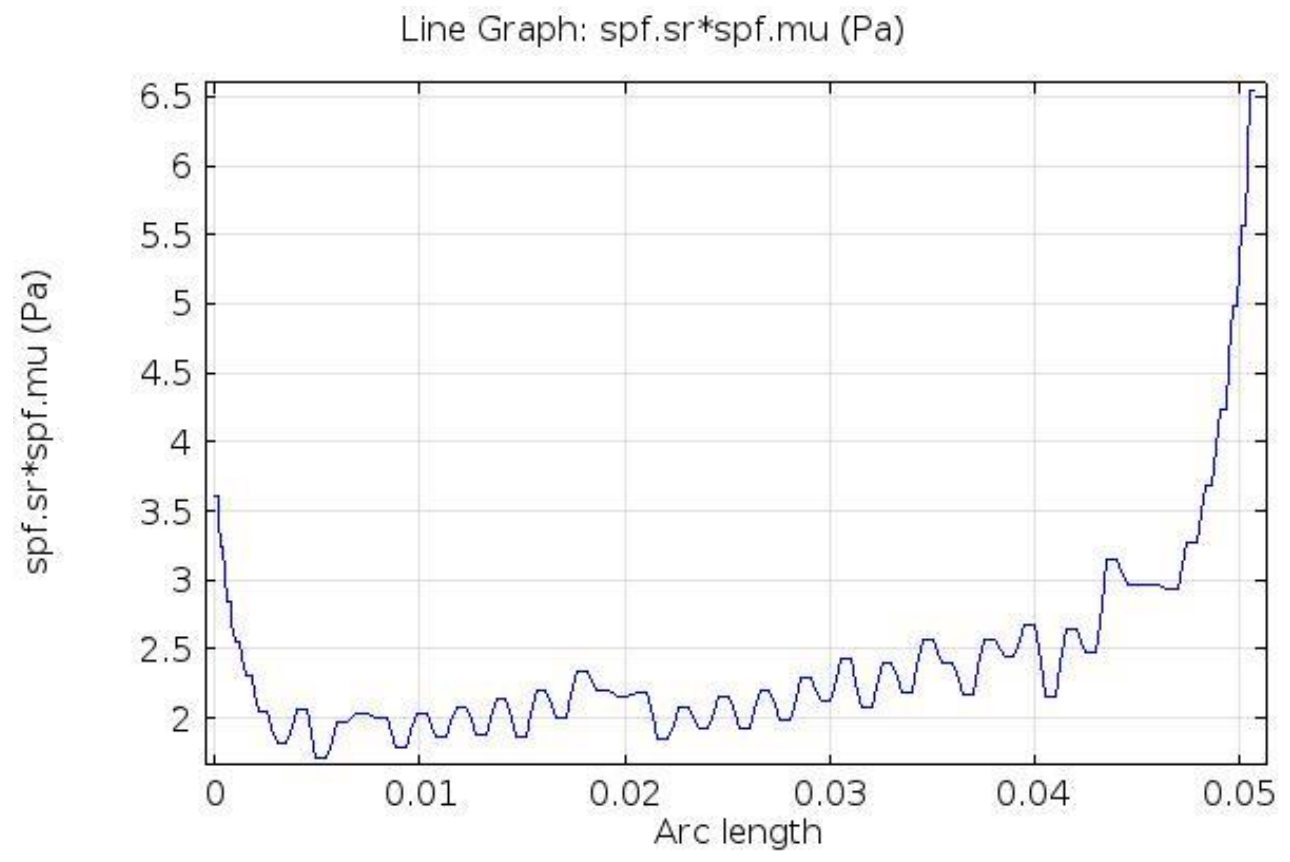

Figure 3.16: Line graph of the shear stress that occurs along the inner edge of the vessel in the non-aneurysmal vessel 


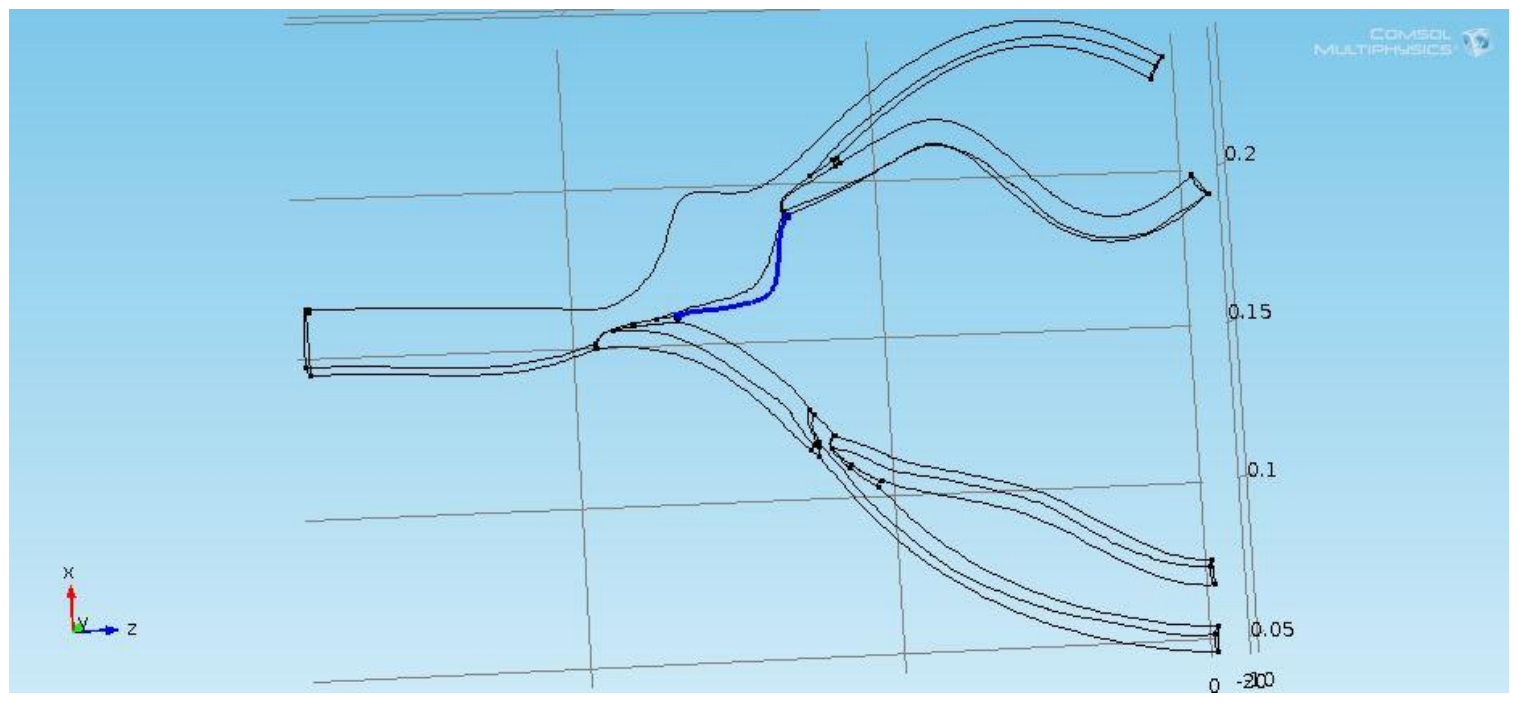

Figure 3.17: Blue line depicting the inner edge of the vessel from which the shear stress data was extracted

Line Graph: spf.mu*spf.sr (Pa)

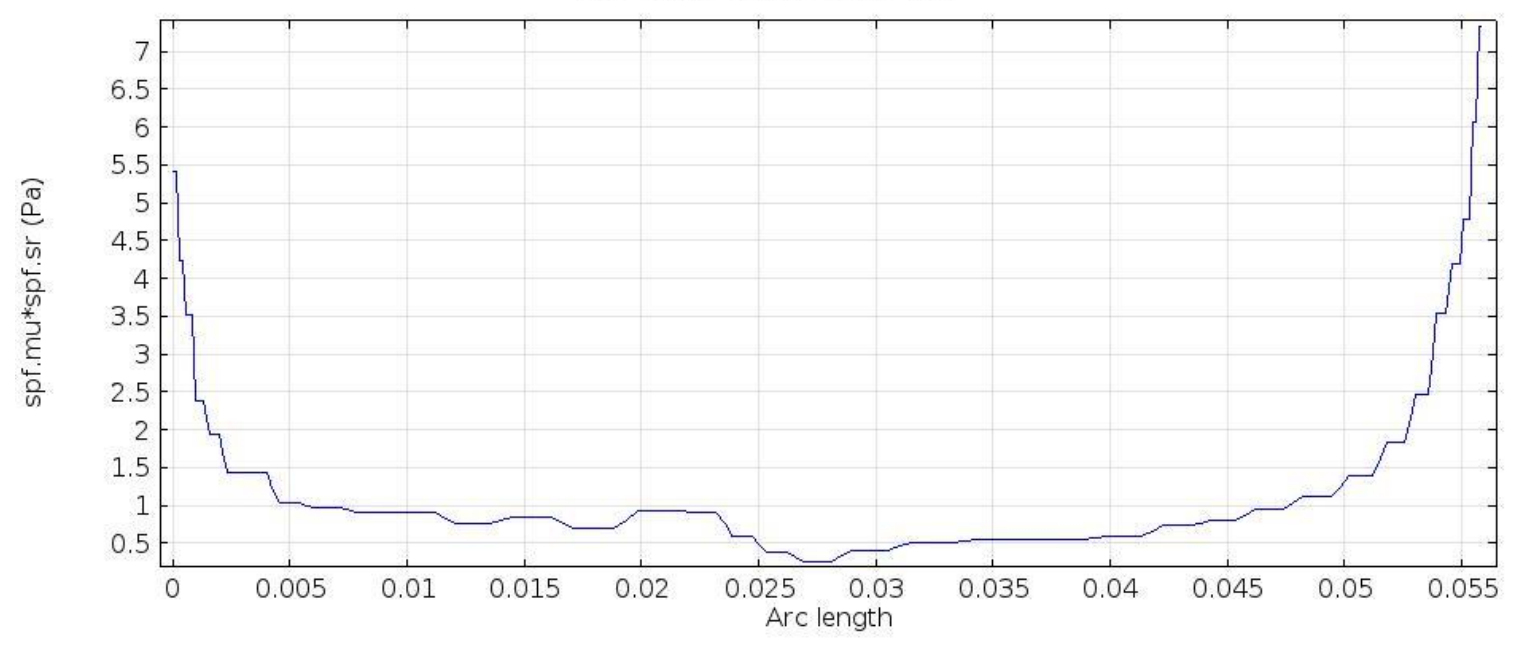

Figure 3.18: Line graph of the shear stress that occurs along the inner edge of the vessel in the aneurysmal vessel

It can be noted from this simulation that the aneurysmal vessel experiences less shear stress compared to the non-aneurysmal vessel. Although the difference is still nominal, small changes in mechanical properties can have serious effects on vessel remodeling. This might lead one to conclude that an aneurysmal vessel is much less likely to grow; however, this simulation does not take into account the weakening of the walls in the vessel. The aneurysmal vessel has already been stretched and therefore has weaker 
wall integrity; therefore, smaller shear stresses will still have a very large impact on the growth and potential rupture of the vessel. The non-aneurysmal vessel can withstand much more shear stress without deformation because the integrity of the wall is still intact. One other conclusion that can be made from these shear stress edge analyses is that the shear stress exponentially increases as the vessel approaches the iliac bifurcation and at the point of entry near the aortic bifurcation. This indicates that sites proximal to the bifurcations can be considered common breeding ground for aneurysms.

The final quantitative data that was collected from the COMSOL MULTIPHYSICS $®$ study was velocities and shear stresses along the cross-section of the aneurysm. This data was used to compare the COMSOL MULTIPHYSICS $®$ model to the empirical model. A cut line was used to collect a line plot and point evaluations at the horizontal centerline of the aneurysm.

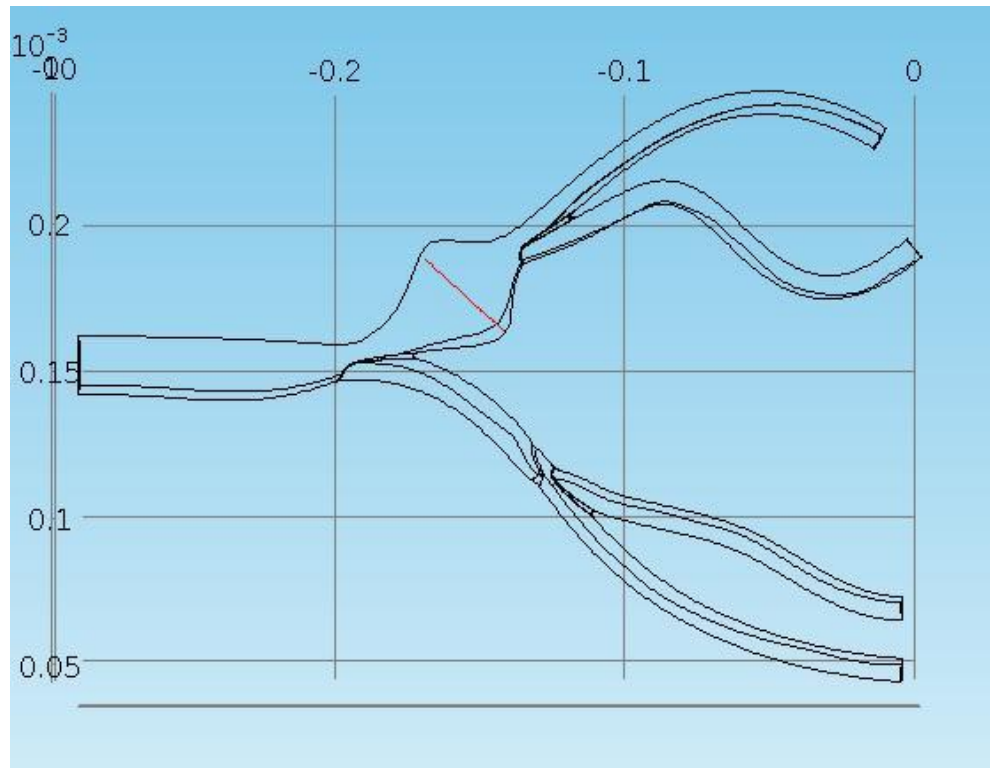

Figure 3.19: Red line indicating the cut line from which the following data was extracted 
Line Graph: Velocity magnitude (m/s)

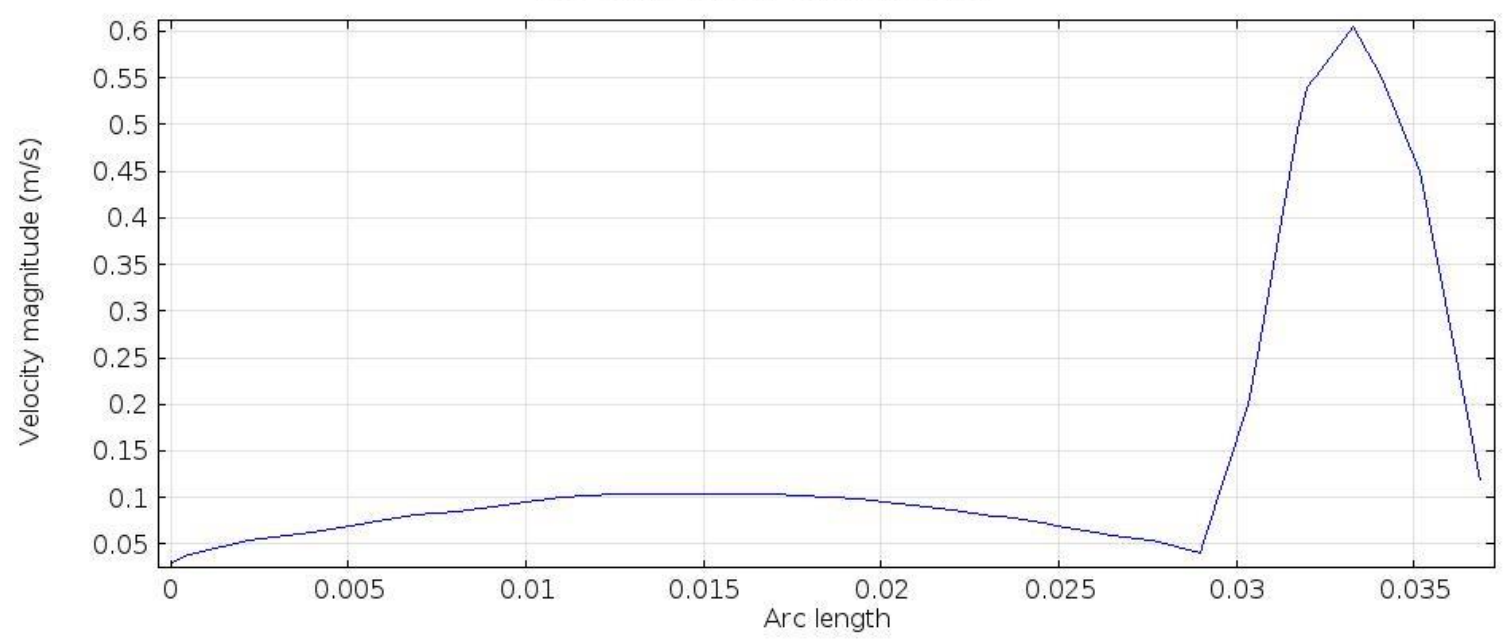

Figure 3.20: Line graph of the velocity across the centerline of the aneurysm Line Graph: spf.mu*spf.sr (Pa)

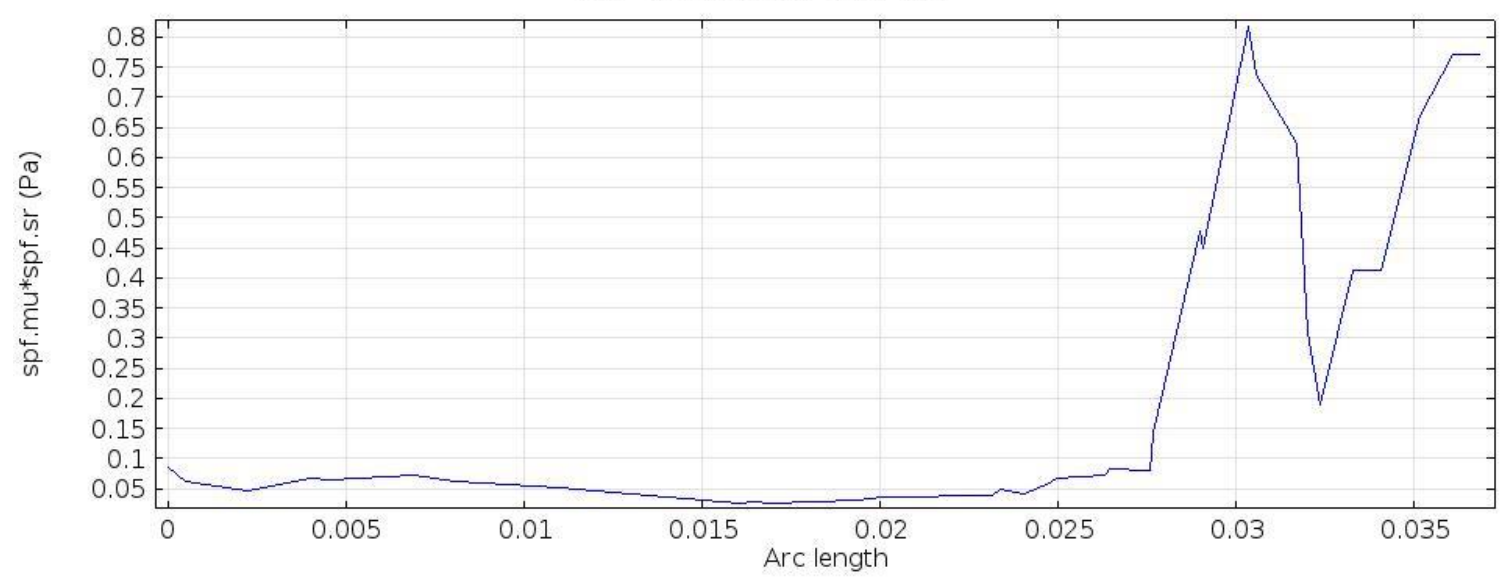

Figure 3.21: Line graph of the shear stress across the centerline of the aneurysm

The graphs follow a similar pattern, indicating that the higher the velocity, the higher the shear stress. This can be verified by Newton's equation for shear stress. Where tau is the shear stress, mu is the dynamic viscosity and $u$ is the velocity.

$$
\tau=-\mu \frac{\partial v}{\partial r}
$$

It can also be seen that there is a dramatic increase in velocity and shear stress on the medial side of the branch; this can be because the line of action follows along the medial 
side of the aneurysm, and this is what is essentially causing the vortex. These velocities can be used to create a velocity profile and derive further fluid dynamic properties.

\subsubsection{Particle Tracking}

The particle tracking simulation showed comparable results to both the empirical model and the laminar COMSOL MULTIPHYSICS $®$ simulation. The main feature of the particle tracking simulation is the back flow and vortex captured in the aneurysm. This simulation clearly demonstrates the lack of fluid flow reaching the outer walls of the aneurysm. Figure 3.23 captures the particle tracking simulation 1 second after the first release of particles and as can be seen from this screen shot there is are no particles touching the upper outside edge or center of the aneurysm.

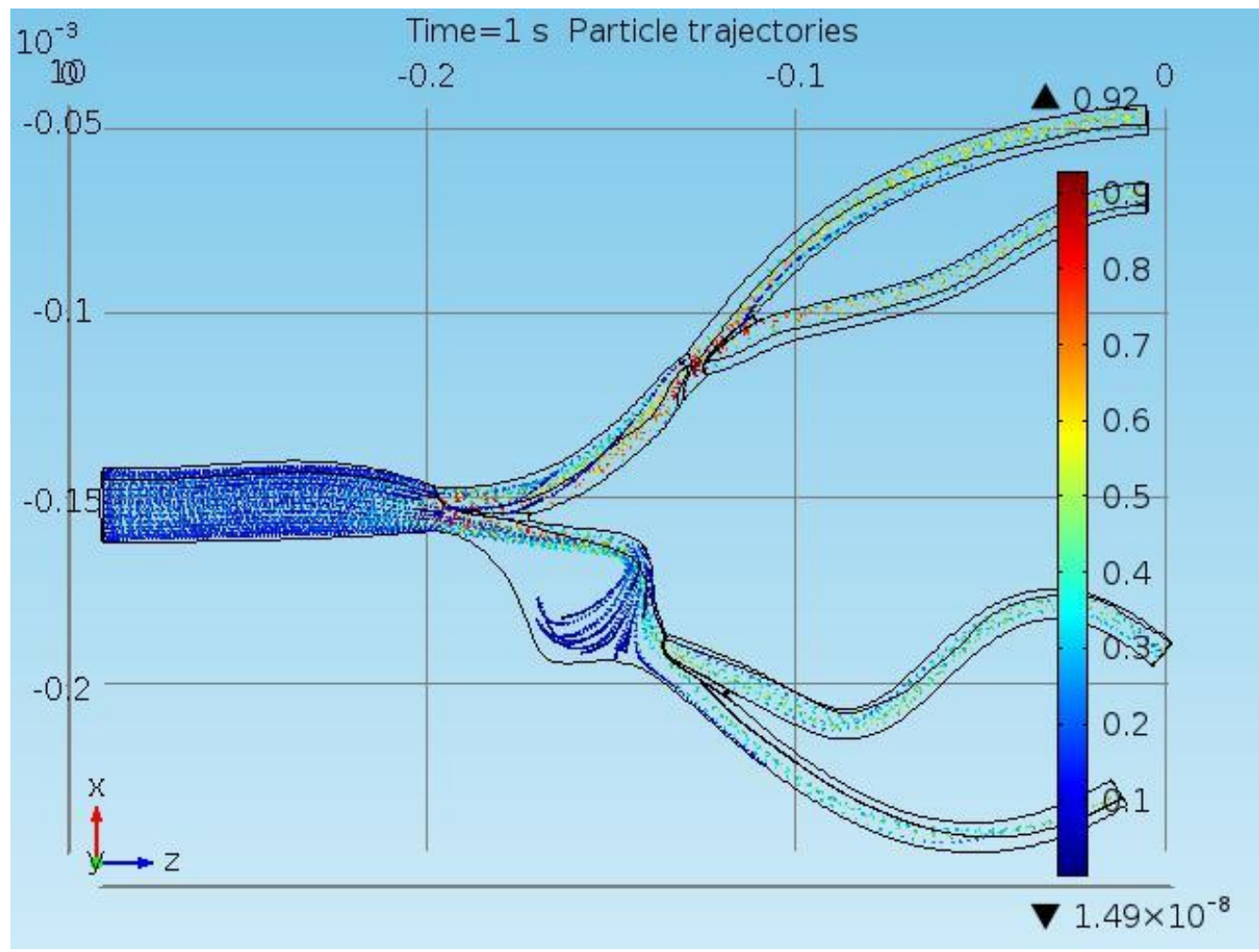

Figure 3.22: Particle tracking simulation at 1 second after initial particle release 
This could potentially indicate complications in remodeling from the lack of exposure of stimuli to the endothelial cells lining that region of the vessel.

\subsection{Comparative Results}

The following graph is a comparative analysis of the velocities found experimentally and through the COMSOL MULTIPHYSICS ® simulation along the path of the fluid flow over time. The velocities for the experimental model were calculated by measuring the distance between the furthest downstream point of dye in one frame and the furthest downstream point of dye in the preceding frame. That distance was then divided by the time between frames. The velocities from the COMSOL MULTIPHYSICS $₫$ model were calculated using point evaluations at coordinates matched to the empirical model.

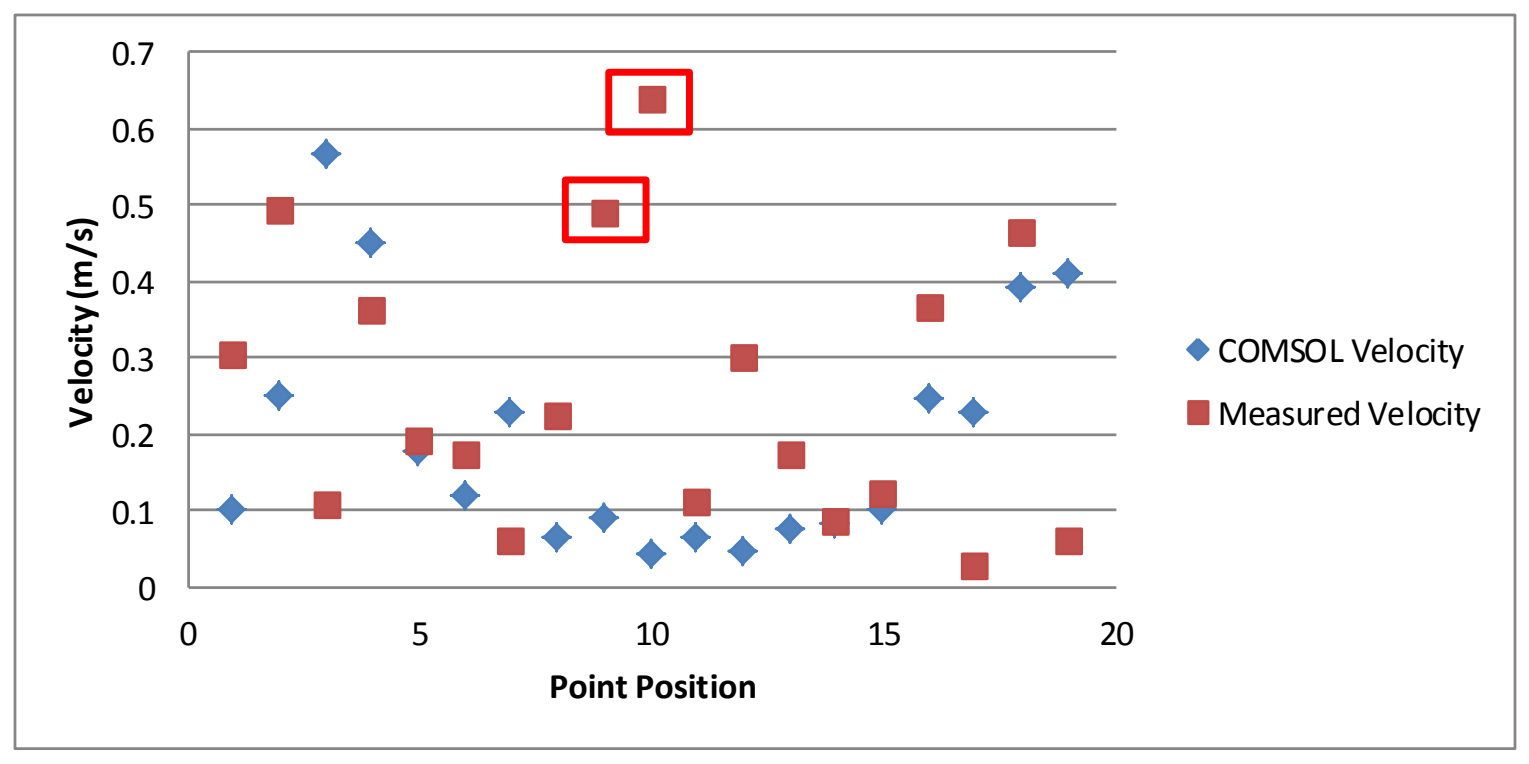

Figure 3.23: Velocity $(\mathrm{cm} / \mathrm{s})$ comparison of the experimental findings and the COMSOL MULTIPHYSICS ${ }^{\circledR}$ findings. Outliers outlined in red

This graph shows disagreement towards the inlet of the vessel (frame 0 to frame 10). Hypotheses that may explain this phenomenon can be found in the discussion chapter. Beyond frame 10, the velocities measured from the empirical model, roughly approach 
the velocities derived from the COMSOL MULTIPHYSICS $\AA$ simulation following the same trend in velocity magnitude. Although the correlation appears to be insignificant, a Paired T-Test and a alpha value of 0.05 found that there was not enough evidence to conclude a significant difference in the velocity values measured through the experiment and the velocity values produced in COMSOL MULTIPHYSICS $\AA(p=0.348)$. 


\section{DISCUSSION}

\subsection{Interpretation of In Silico Findings}

The outcome of the COMSOL MULTIPHYSICS ® simulation allowed for a composite of results providing both numerical and graphical data. Figure 3.10 shows a directional arrow plot which shows both the direction and comparative magnitudes of the fluid flow through the aneurysm. This shows the development of a vortex and which walls are going to receive the most impact from fluid velocity. Figure 3.4 and 3.5 is another graphical result showing the magnitudes of the velocity along a color gradient. This image shows extremely high velocities superior to the aneurysm and along the internal wall of the aneurysm. This is to be expected as the volume of the artery slightly decreases as the aorta ends and the common iliac artery begins. Another significant fluid dynamic change observed as a result of the aneurysm is the difference in velocity magnitudes in the external and internal branches of the iliac artery with the aneurysm, and the vessel without the aneurysm. The external and internal iliac arteries downstream of the aneurysm experience much lower velocities than their counterparts on nonaneurysmal vessel. This could result in poor circulation distal to the aneurysm as well as less oxygen supply. The final image generated in COMSOL MULTIPHYSICS ® , figure 3.6 and 3.7 shows the shear stress along a color gradient. Most of the vessel experiences low shear stresses, generally between 10 and 40 dynes $/ \mathrm{cm}^{2}$ (Figure 4.1). 


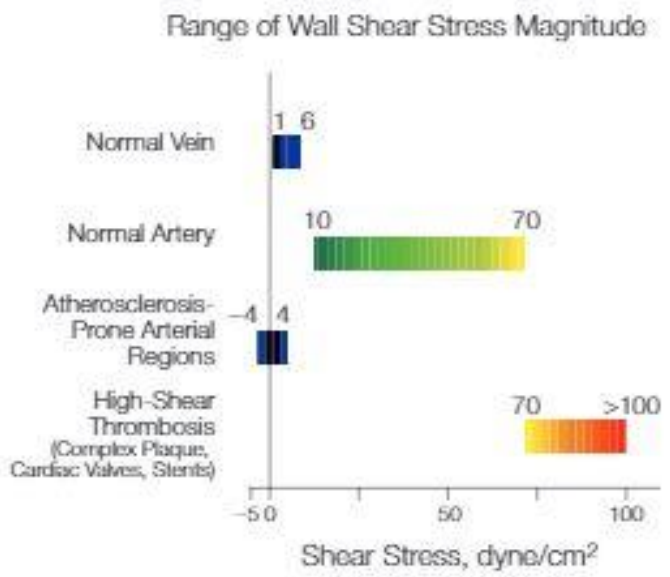

Figure 4.1: A range of shear stress magnitudes encountered in veins, arteries, and in low-shear and high-shear pathologic states

The highest shear stress occurs at the bifurcation and the lowest shear stress occurs on the lateral interior of the aneurysm. After comparing the non-aneurysmal vessel to the aneurysmal vessel, the aneurysmal vessel showed a significant decrease in shear stresses along the walls. The low shear stresses can result in atherosclerosis [11]. In numerous experiments, shear stress has been shown to influence vessel wall remodeling. Specifically, chronic increases in blood flow, and consequential shear stress, lead to expansion of the luminal radius such that mean shear stress is returned to its baseline level [11]. Conversely, decreased shear stress, as seen on the lateral wall of the aneurysm, resulting from lower flow or blood viscosity, can cause a decrease in internal vessel radius. The net effect of these endothelial-mediated compensatory responses is the maintenance of mean arterial hemodynamic shear stress magnitude [11]. 


\subsection{Interpretation of Experimental Findings}

The statistical analysis comparing the COMSOL MULTIPHYSICS ${ }^{\circledR}$ simulation velocity values to the experimental velocity values confirmed the accuracy of the empirical study. The vortex observed in the aneurysm is a realistic result of the geometry. As fluid flow enters the aneurysm, it goes from a small fixed volume almost immediately to a much larger volume. A vortex is advanced by the flow across the aneurysm neck and impinges on the distal wall. Another artifact of the experimental model is the proven success of imaging velocity profiles through an iliac aneurysm using food dye. The imaging technique used for this experiment can be considered a novel method. The combination of the organic shape and behavior of flow from the food dye and the concrete numerical data gathered from the threshold adjusted images using ImageJ allowed for more complete results. After analyzing the data from both the half vessel model and the full vessel model, it can be concluded that the half vessel model is a more accurate tool for imaging. The half vessel model utilizes a single plane for imaging which gives a higher resolution image and a more finite velocity profile.

\subsection{Limitations}

\subsubsection{Limitations of the COMSOL MULTIPHYSICS ® Simulation}

As with all finite element simulations such as COMSOL MULTIPHYSICS ${ }^{\circledR}$, there will be limitations to the accuracy of the simulation. In COMSOL MULTIPHYSICS $®$, when a mesh size is assigned, a number of elements are created to make up the geometry, the element size, which (seen in table 2.3), correlates to the resolution of the results: more elements result in a higher resolution. The mesh refinement study was competed using general physics, further mesh refinement in the fluid dynamics category was not explored. Another limitation associated with the COMSOL MULTIPHYSICS ® simulation 
run for this study is the degree at which the material was mimicked. For the purposes of this study, the only material properties that were programed were the density and viscosity of the fluid, in this case, water. No material for the walls was selected which left room for error in the boundary conditions.

\subsubsection{Limitations of the Mechanical Experiment}

The most significant limitation associated with the empirical experiment was that a uniform velocity profile could not be achieved at the inlet; a condition that COMSOL MULTIPHYSICS $®$ assumes. The lack of uniform inflow was partially corrected by adding a conical attachment to the inlet superior to the aorta, however, a perfectly uniform velocity profile was not achieved at the inlet. This limitation caused air bubbles to develop throughout the vessel, an artifact that would not be seen in COMSOL MULTIPHYSICS ${ }^{\circledR}$ or in vivo. These air bubbles were minimized by rocking the vessel back and forth, however, a significant congregation of air bubbles remained at the entrance of the aneurysm. One more limitation to the mechanical experiment were the ridges and seams developed as a result of machining and manufacturing error. FMD creates layers as it builds, as seen in the Methods Chapter, this leaves ridges along the walls of the vessel, a feature that was not mimicked in COMSOL MULTIPHYSICS $®$. Although a significant improvement was made to mitigating the ridges with the use of the finishing coating mentioned in the Methods section, there were still some ridges that could not be sealed. The ridges could result in a change in shear stresses along the wall and cause slower velocities throughout the vessel. The large seam that was created by joining the two vessels was a major limitation to the study. In order to create the full vessel, the accuracy along the centerline of the walls had to be forfeited. The seam would essentially cause the same effect as the ridges, however unlike the ridges, the 
direction of the seam matched the direction of flow, and therefore a large decrease in shear stress along the walls was not witnessed.

\subsubsection{Study Limitations}

There were many limitations that differentiated this study from an in vivo analysis of an iliac aneurysm. There was no pulsatile flow mimicked in either the mechanical study or

the COMSOL MULTIPHYSICS ® study, a feature that is exhibited by the heart and plays an important role in the fluid properties and propagation. The use of water instead of blood or a blood-like fluid in the in-vitro model left room for errors in viscosity differences, the absence of rouleaux formation and any coagulation. The tortuosity and anatomy of the vessel was arbitrary and created based on a literature review rather than a CT Scan or MRI image. Atherosclerosis was not accounted for in either models, a disease often associated with CIAs. Atherosclerosis could result in a different interior geometry of the vessel and might play a role in the fluid properties, therefore it should be considered in future iterations. The final limitation of the study was that the walls of the study in either model did not mimic the true physiology of a vessel wall. There was no accounting for endothelial tissue lining the wall which plays a large role in the fluid dynamics of the natural vessel. The assumption of laminar flow can also be considered a limitation. The Reynolds number used for this study assumed laminar flow in a smooth pipe, however, in the empirical model, ridges, seams, and tears can cause viscous differences in the flow, transitioning the Reynolds number more towards turbulent flow.

\subsection{Conclusions and Future Iterations}

Iliac aneurysms are difficult to treat, however, with an accurate computer simulation and a functioning in-vitro vessel, advances in treatment technology can be made. In future iterations, the creation of a full vessel computer model that is able to show the 
deformation pattern of the vessel could provide more valuable information on the propagation of the aneurysm. Deformation studies also allow the user to see the effects of different anatomical properties on the propagation of the aneurysm over time such as wall strength, ischemia, viscosity and plaque formation. COMSOL MULTIPHYSICS ${ }^{\circledR}$ has the capability to analyze fluid on wall interactions and quantify the deformation over time. Abaqus also has the capability to perform deformation studies. Both of these softwares can even allow the user to input a threshold for bursting, essentially p redicting the conditions under which the vessel will burst. Future iterations could also achieve a 3D model with virtually no ridges, which was an artifact from the FMD; this might include more advanced polishing techniques or higher definition FMD technology. One other future study that should be considered is a threshold study to evaluate the critical shear stress rate that clot formation occurs. All of the future iterations could provide dramatic clinical impact with regard to both predicting aneurysm rupture and treatment solutions. Improvements to this study can be made by adjusting for any of the other limitations mentioned in the Limitations section. In conclusion, a full vessel COMSOL MULTIPHYSICS $®$ model provides an accurate tool for analyzing the fluid properties in an aneurysm and the full vessel empirical model would be a good candidate for accurately experimenting with iliac artery aneurysm treatments and prevention. 


\section{REFERENCES}

[1] M. Nagarajan, P. Chandrasekar, E. Chandrasekar, and S. Muralidharan, "Repair of iliac artery aneurysms by endoluminal grafting: The systematic approach of one institution," Tex Heart Inst J., vol. 27, pp. 250-252, 2000.

[2] R. S. Sandhu and I. I. Pipinos, "Isolated iliac artery aneurysms," Seminars in Vascular Surgery, vol. 18, pp. 209-215, 2005.

[3] "Blood Vessel Structure." Boundless Anatomy and Physiology. Boundless, 26 May. 2016. Retrieved 29 May. 2016

[4] Lasheras, Juan C. "The Biomechanics of Arterial Aneurysms". Annual Review of Fluid Mechanics 39 (2007): 293-319.

[5] R. A. McCready, P. C. Pairolero, and J. C. Gilmore, "Isolated iliac artery aneurysms," Surgery, vol. 93, pp. 688-693, 1983.

[6] J. W. Richardson and L. J. Greenfield, "Natural history and management of iliac aneurysms," J Vasc Surg, vol. 8, pp. 165-171, 1988.

[7] G. Ailawadi, J. L. Eliason, and G. R. Upchurch, "Current concepts in the pathogenesis of abdominal aortic aneurysms," J Vasc Surg, vol. 38, pp. 584-588, 2003.

[8] Y. C. Fung "Biomechanics: Circulation", 1997.

[9] M. LaBarbera, "Principles of design of Fluid trans-port systems in zoology," Science, p. $249,1990$.

[10] Andreas Raabe, Jurgen Beck. "Intracranial Aneurysms: New Aspects of Formation, Rupture, and Outcome". Schweizer Archive for Neurologie 162.4 (2011): 169-170.

[11] I. Sakamoto, E. Sueyoshi, and S. Hazama, "Endovascular treatment of iliac artery aneurysms," Peripheral Vascular Diagnosis and Interventions, vol. 25, pp. S213S227, 2005. 
[12] Lawrence, Peter F., Shauna Lorenzo-Rivero, and Joseph L. Lyon. "The Incidence of lliac, Femoral, and Popliteal Artery Aneurysms in Hospitalized Patients." Journal of Vascular Surgery 22.4 (1995): 409-16. ScienceDirect. Web. 5 June 2016.

[13] B. Alberts, A. Johnson, J. Lewis, "Blood Vessels and Endothelial Cells" Molecular Biology of the Cell $4^{\text {th }}$ Edition. Garland Science 2002.

[14] A. M. Malek, S. L. Alper, and S. Izumo, "Hemodynamic shear stress and its role in atherosclerosis," JAMA, vol. 282, pp. 2035-2042, 1999.

[15] J. C. Lasheras, "The biomechanics of arterial aneurysms.," Annual Review of Fluid Mechanics, vol. 39, pp. 293-319, 2007.

[16] A. L. Asbury, J. W. Ruberti, E. I. Bluth, and R. A. Peattie, "Experimental investigation of steady flowin rigid models of abdominal aortic aneurysms," Annual Biomedical Engineering, vol. 23, pp. 23-39, 1995.

[17] R. Budwig, D. Elger, H. Hooper, and J. Slippy, "Steady flow in abdominal aortic aneurysm models.," J. Biomech. Eng., vol. 115, pp. 418-423, 1993.

[18] A. C. Salsac, "Changes in the hemodynamic stresses occurring during the enlargement of abdominal aortic aneurysms.," PhD Thesis, University of San Diego, 2005.

[19] E. A. Finol, K. K, and A. C. H., "The effect of asymmetry in abdominal aortic aneurysms under physiologically realistic pulsatile flow conditions.," J. Biomech. Eng., vol. 125, pp. 207-217, 2003.

[20] O. S. Carneiro, A. F. Silva, and R. Gomes, "Fused deposition modeling with polypropylene," Materials and Design, vol. 83, pp. 768-776, 2015.

[21] T. A. Grimm, "Fused deposition modeling: A technology evaluation," T. A. Grimm and Associates inc., 2002.

[22] H. Mayer, "Soft lithography," Lecturer, Department of Mechanical Engineering, California Polytechnic State University, 2015. 
[23] Eddings, Mark A., Michael A. Johnson, and Bruce K. Gale. "Determining the Optimal PDMS-PDMS Bonding Technique for Microfluidic Devices." J. Micromech. Microeng. Journal of Micromechanics and Microengineering 18.6 (2008): 067001. IOP Science. Web. 28 May 2016.

[24] R. L. Fournier, Basic Transport Phenomena in Biomedical Engineering. CRC Press. 


\section{APPENDICES}

APPENDIX A: Still Images from the Time Dependent Study

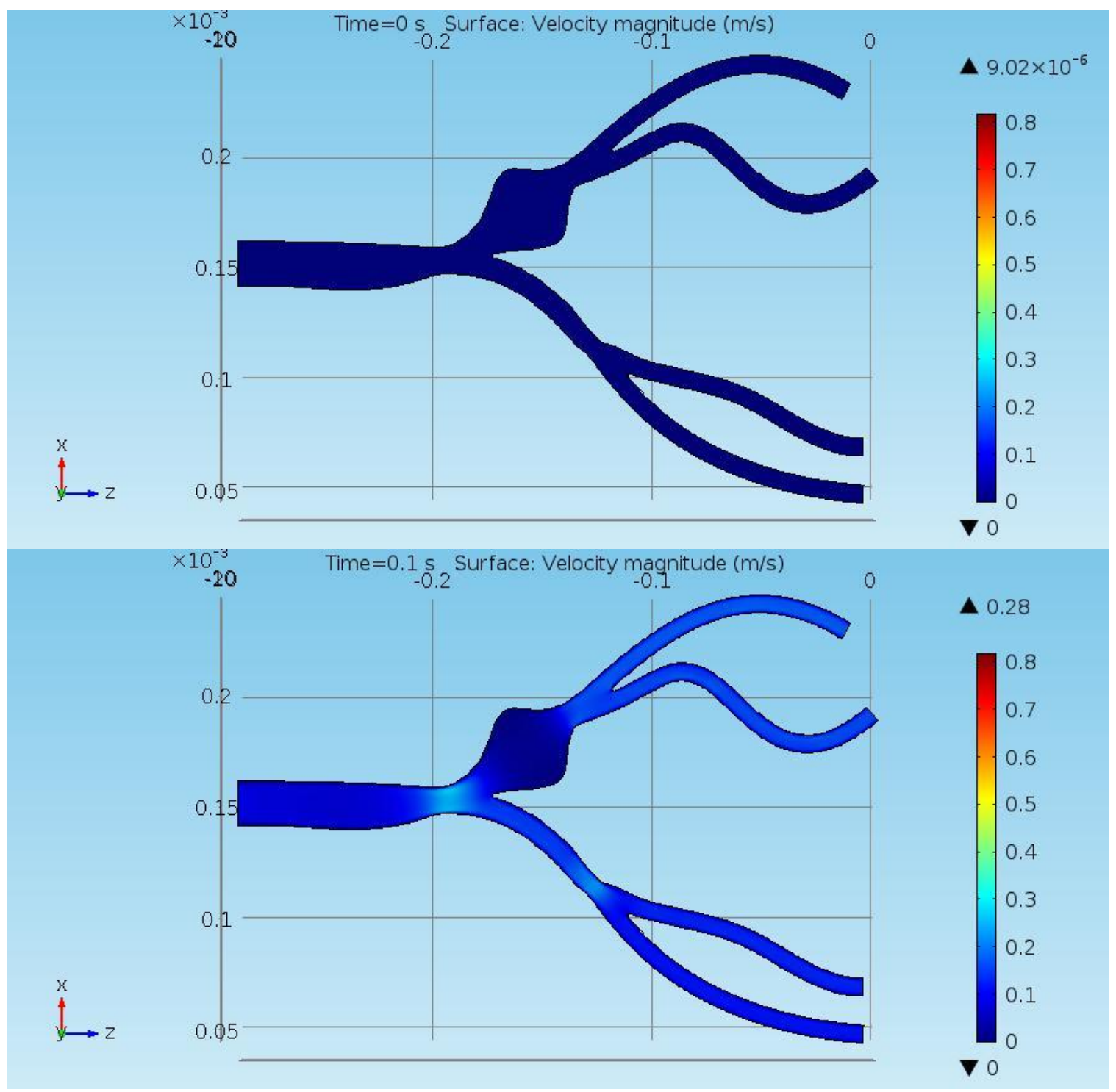




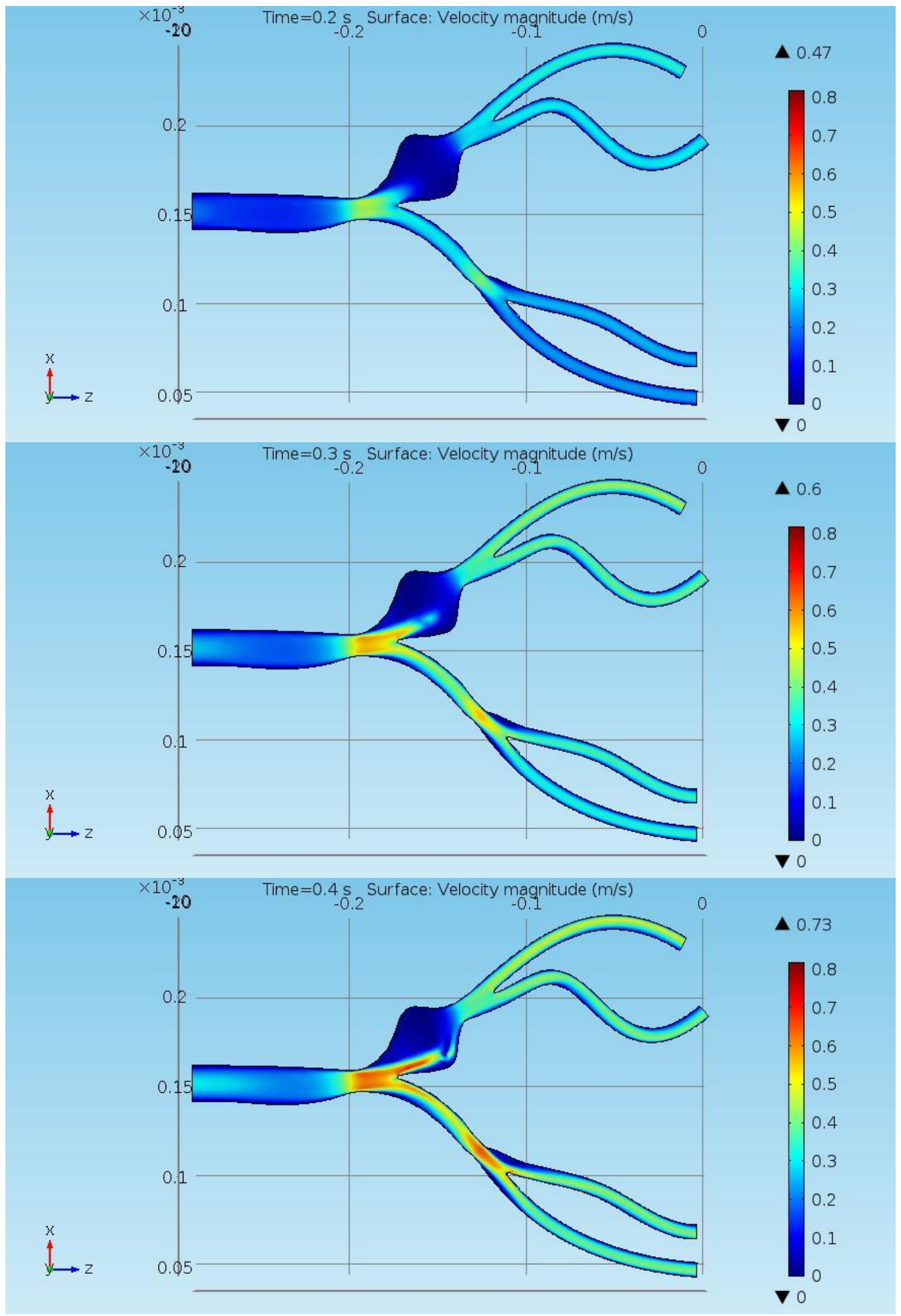




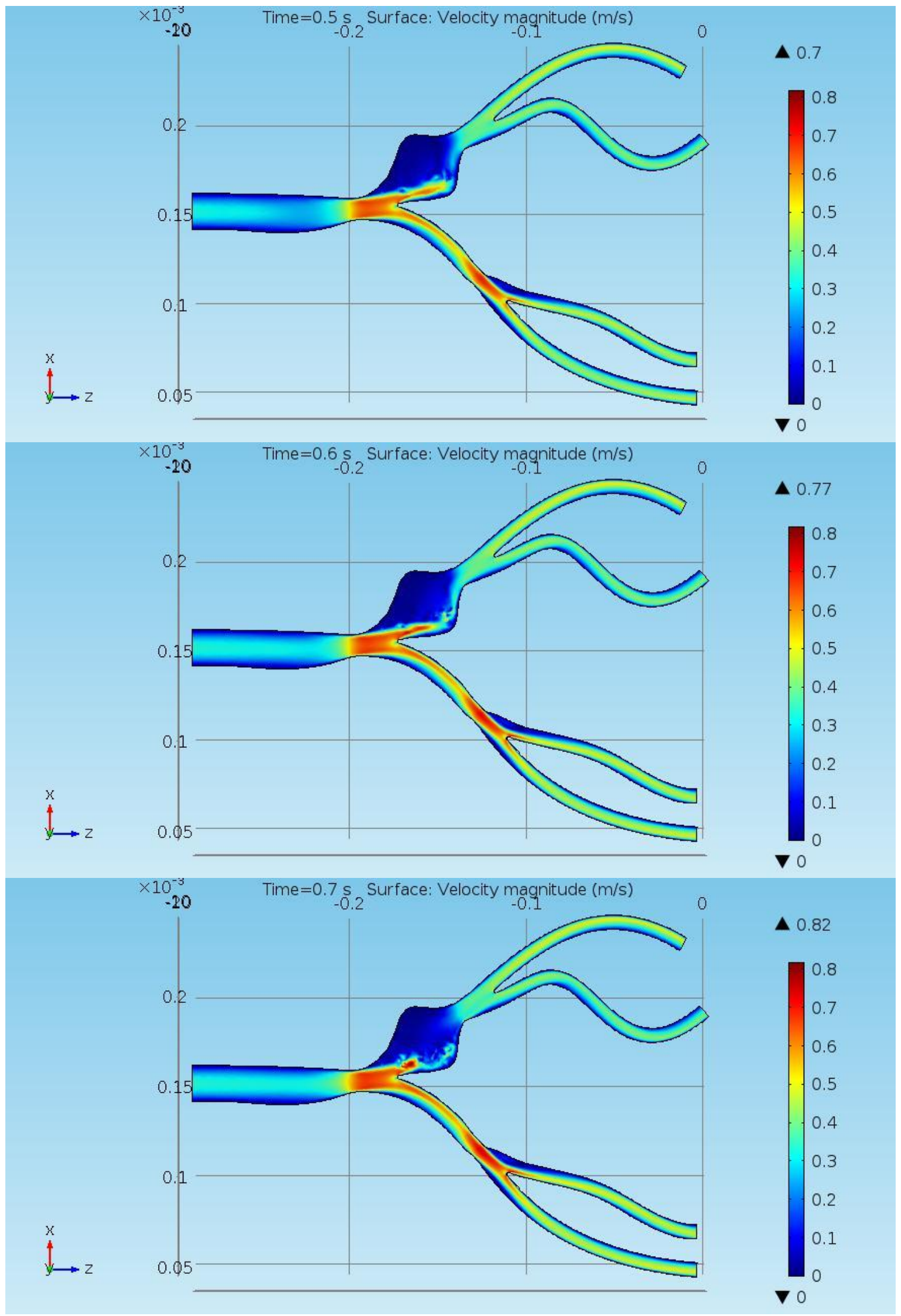




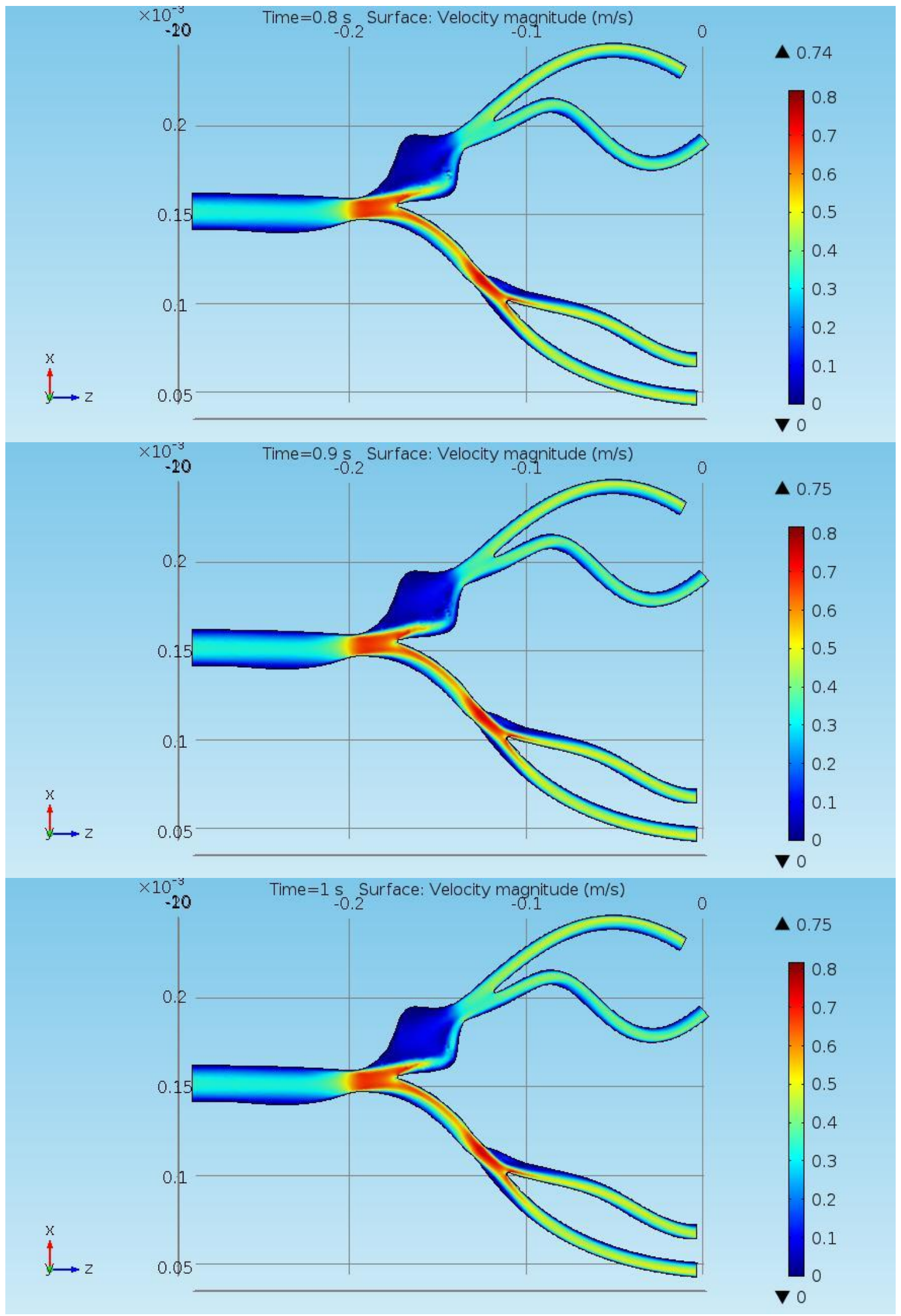


APPENDIX B: Still Images Extracted from the Video of the Empirical Model 


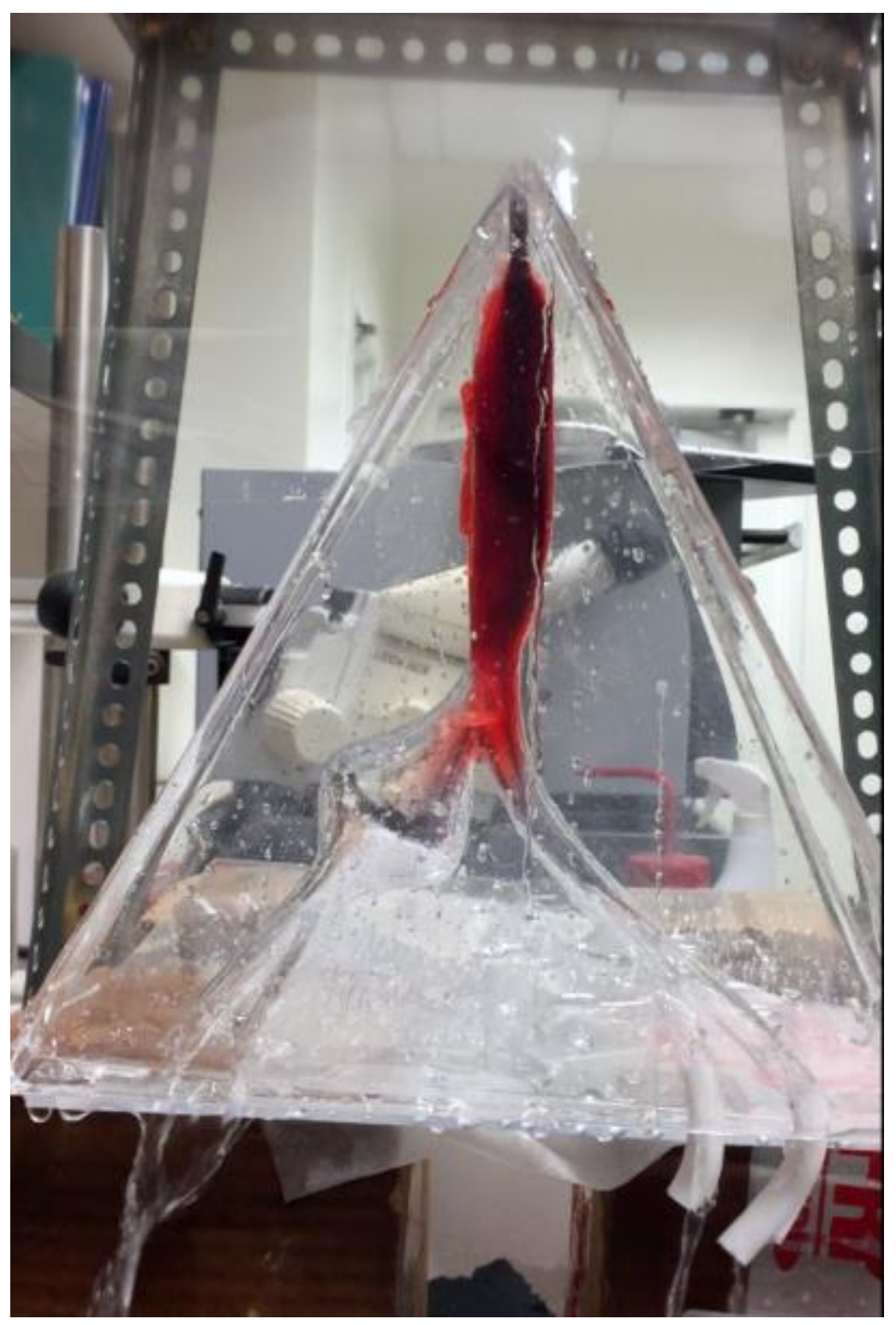




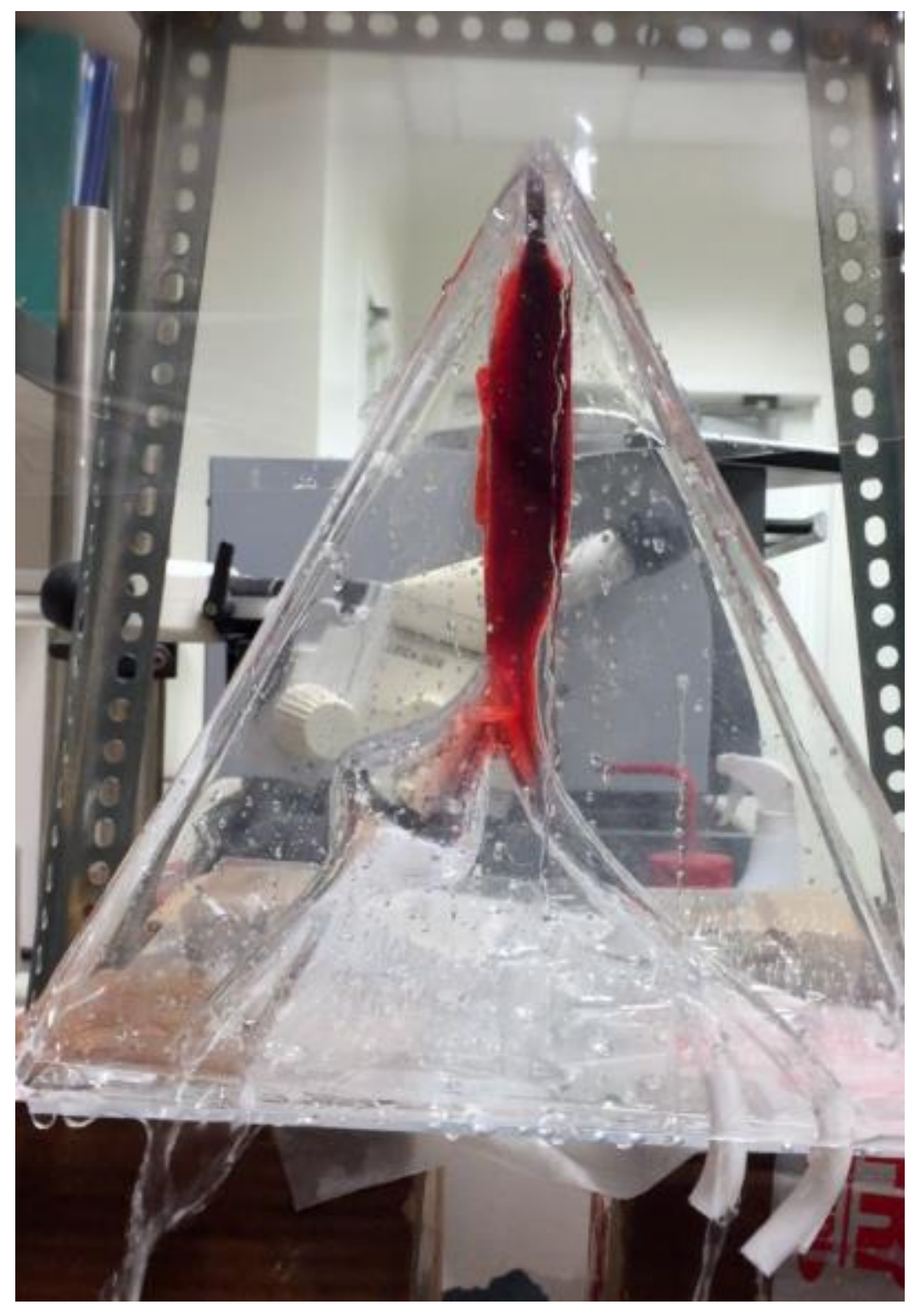




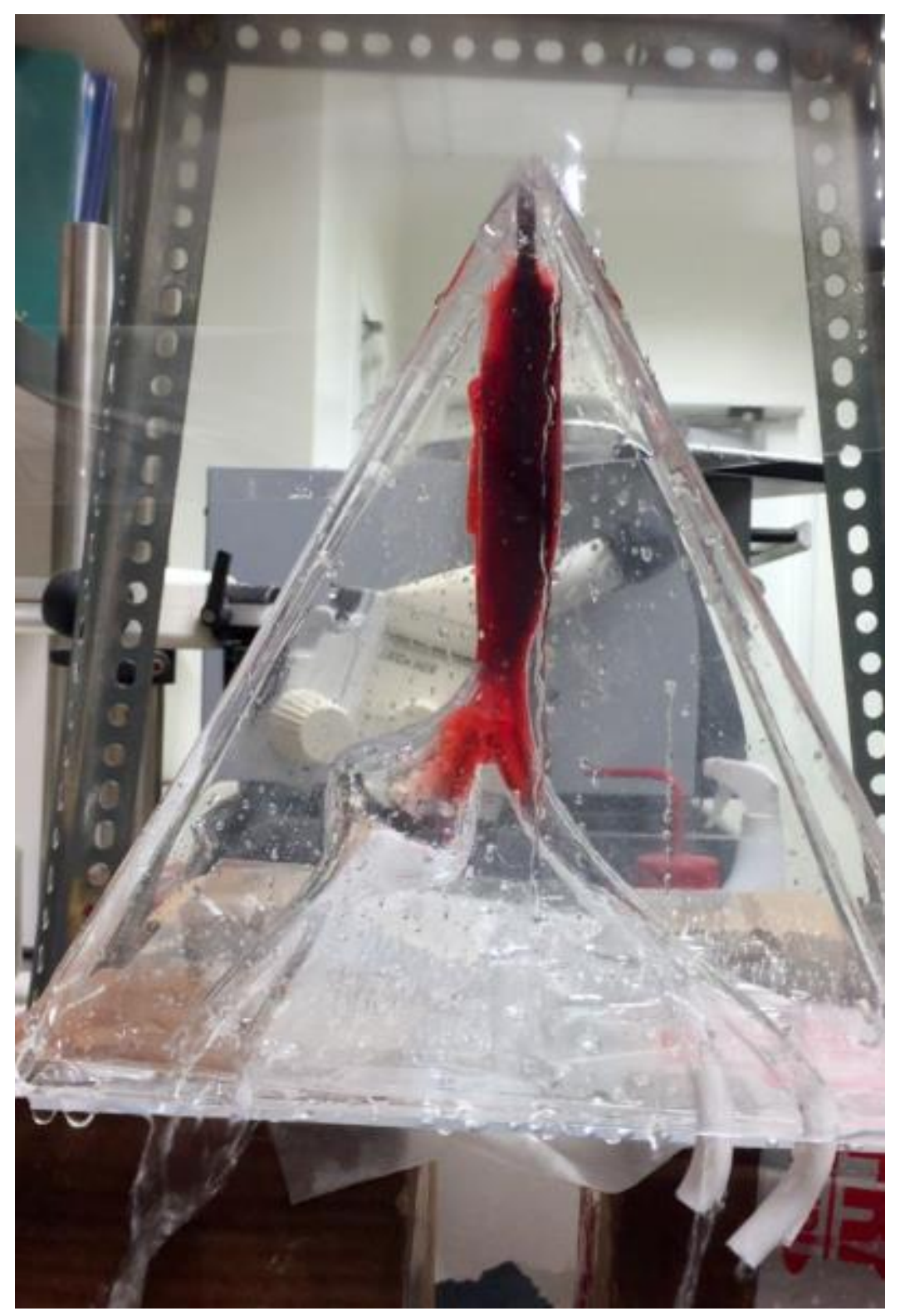




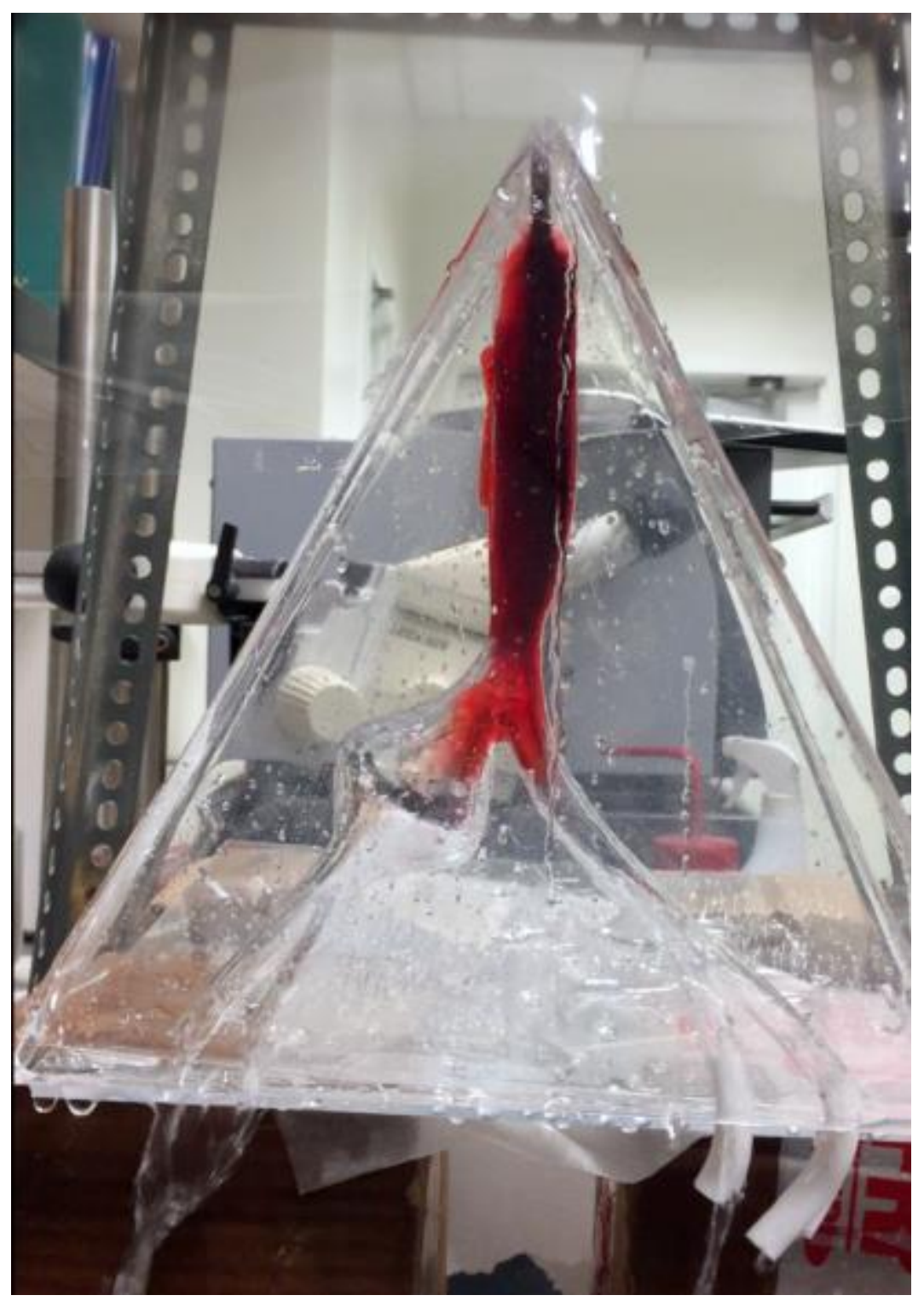




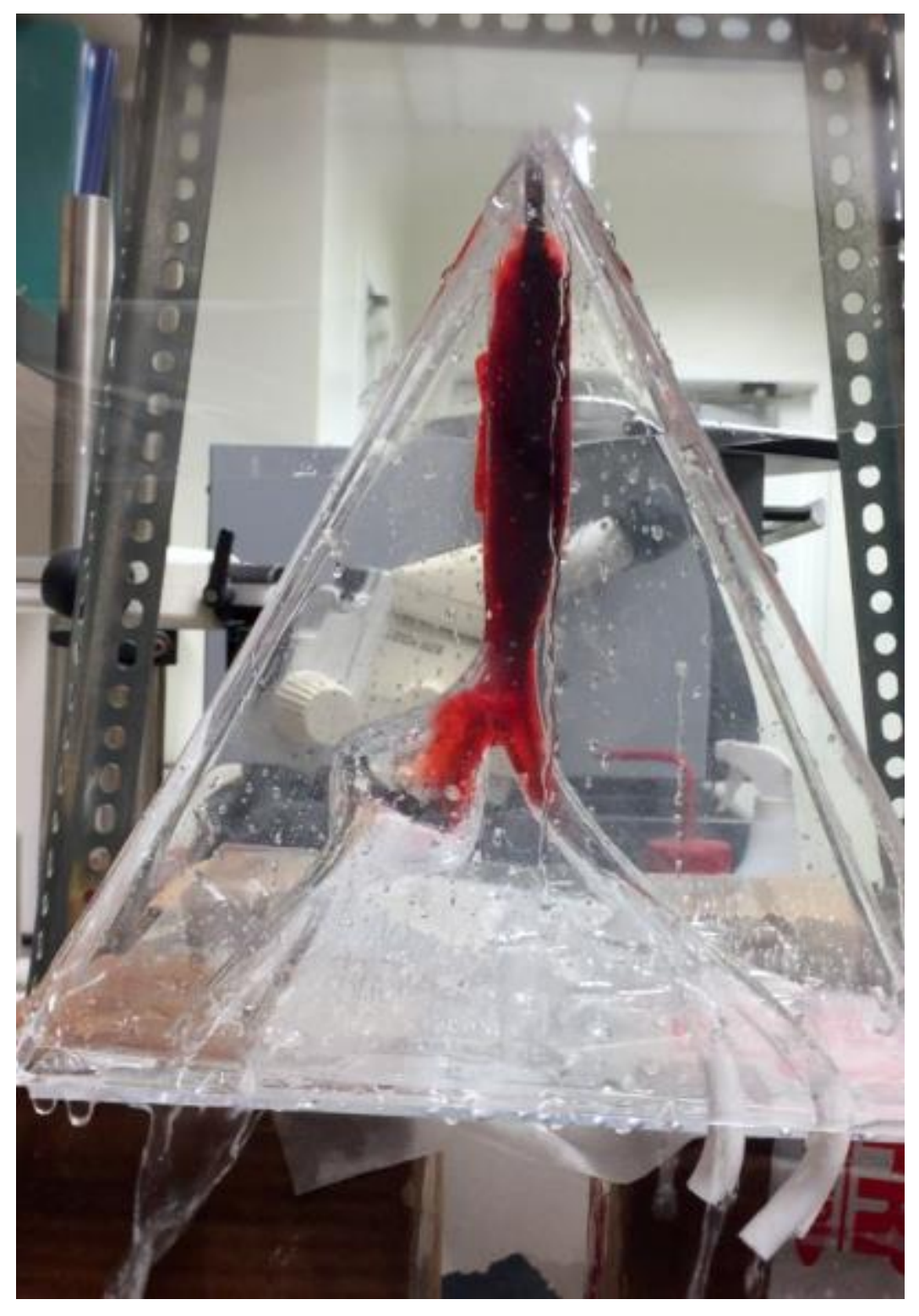




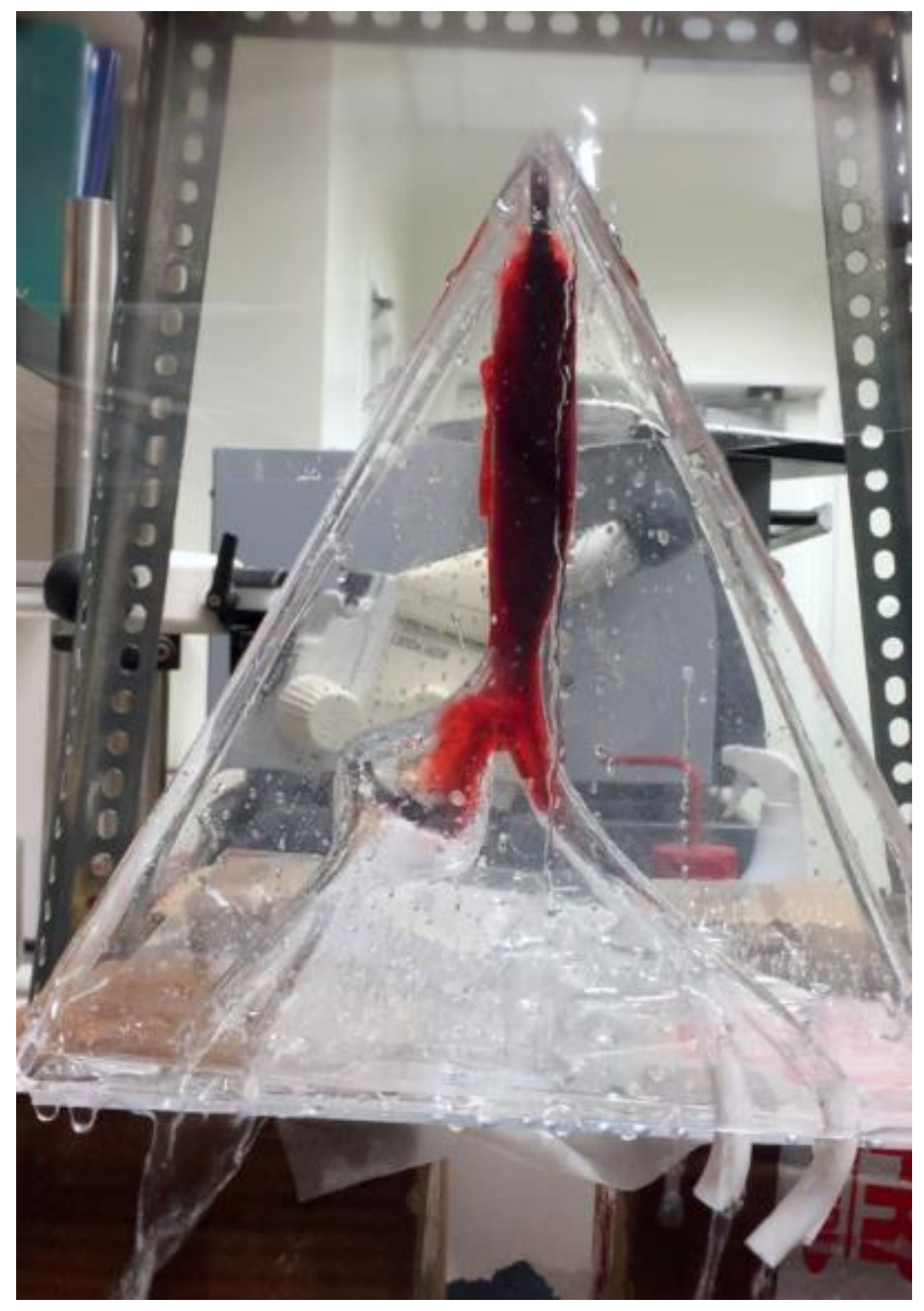




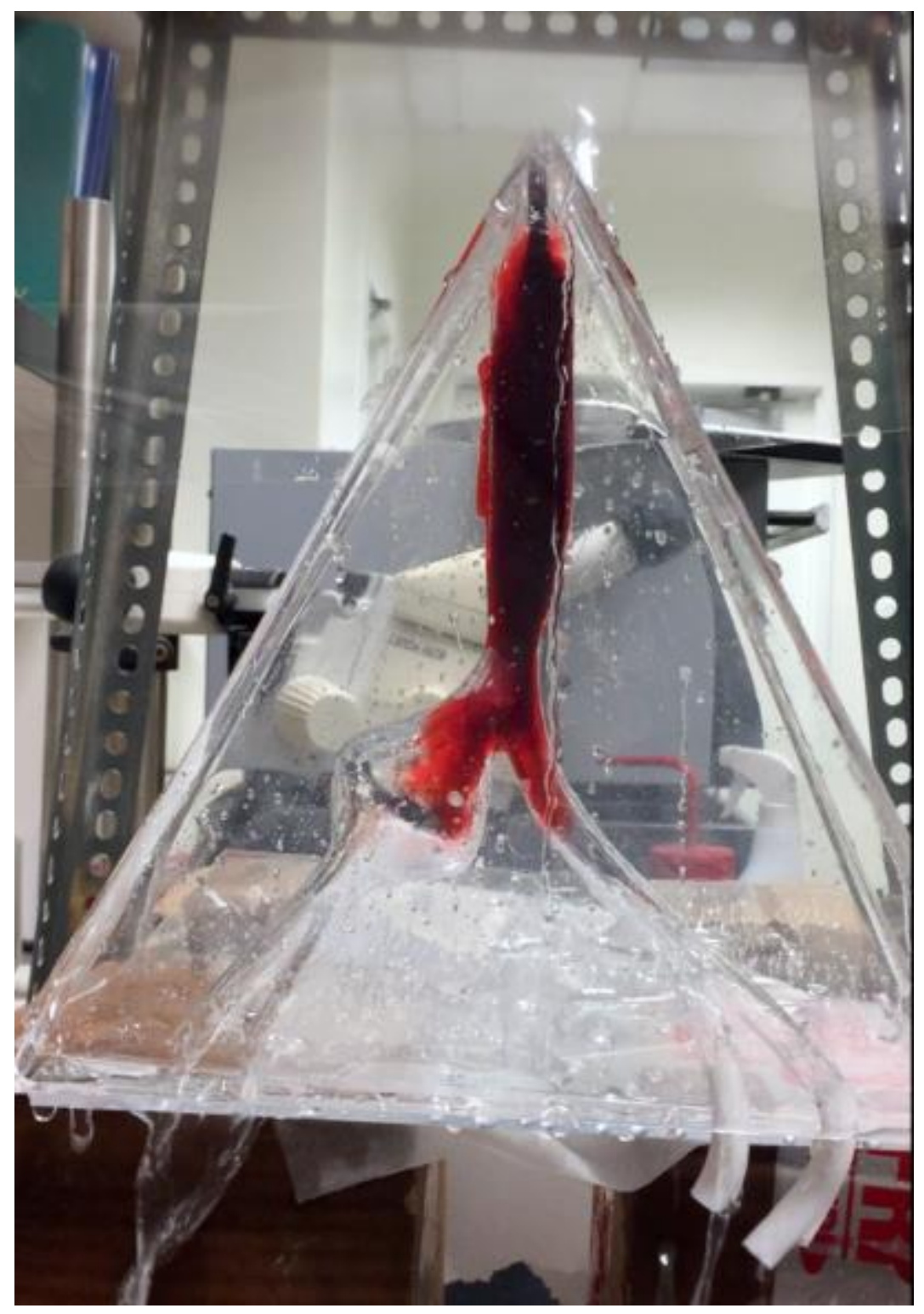




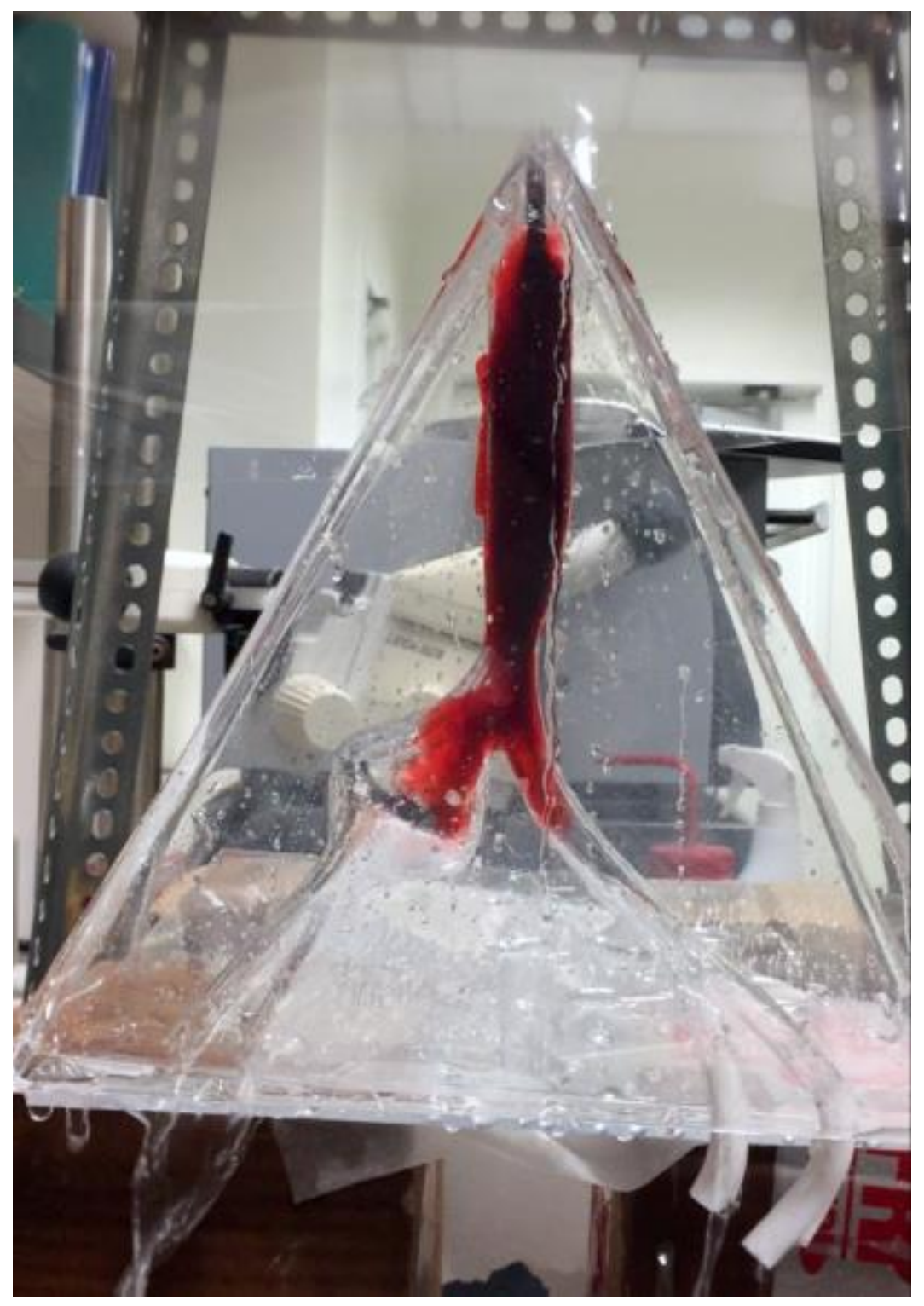




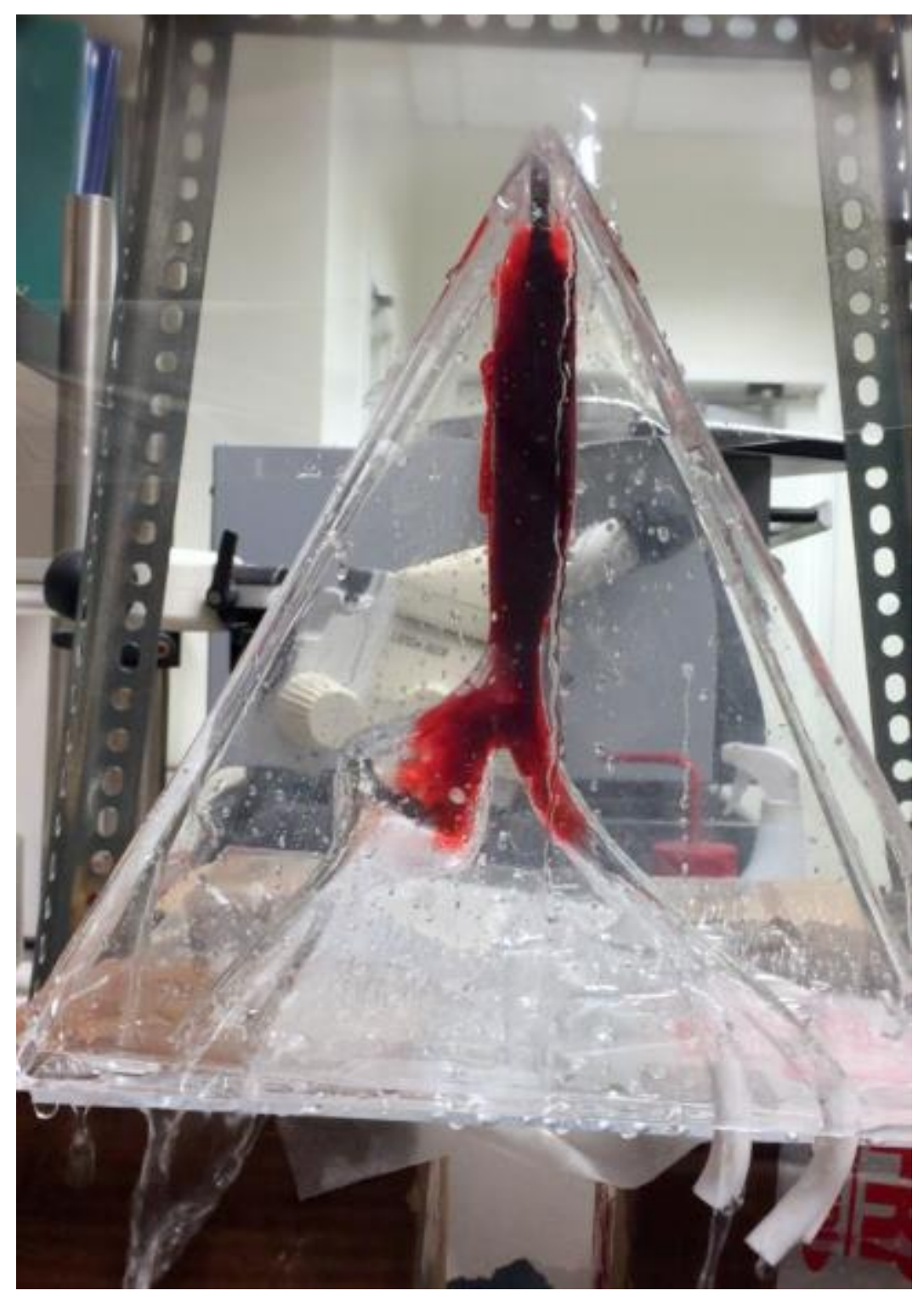




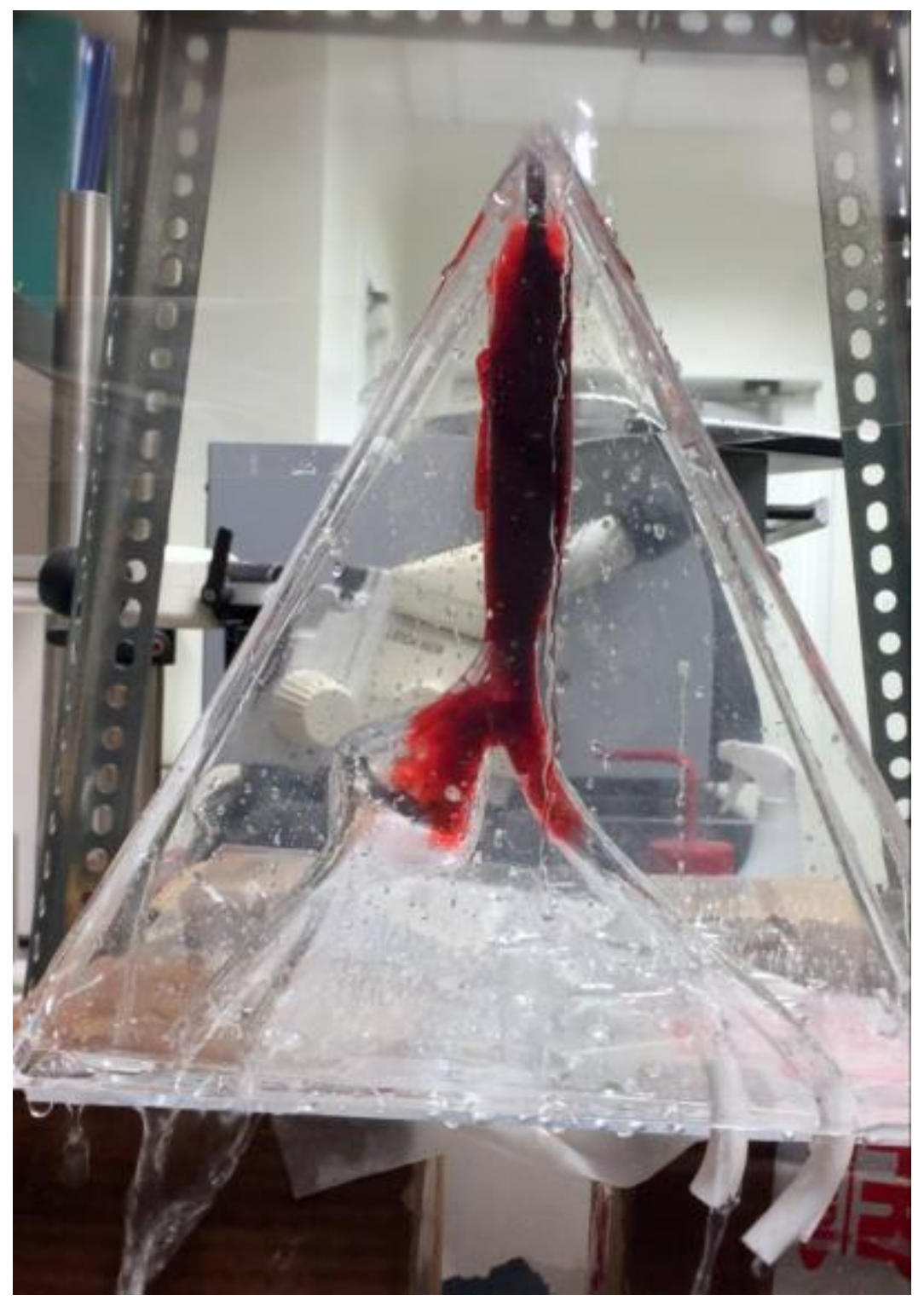




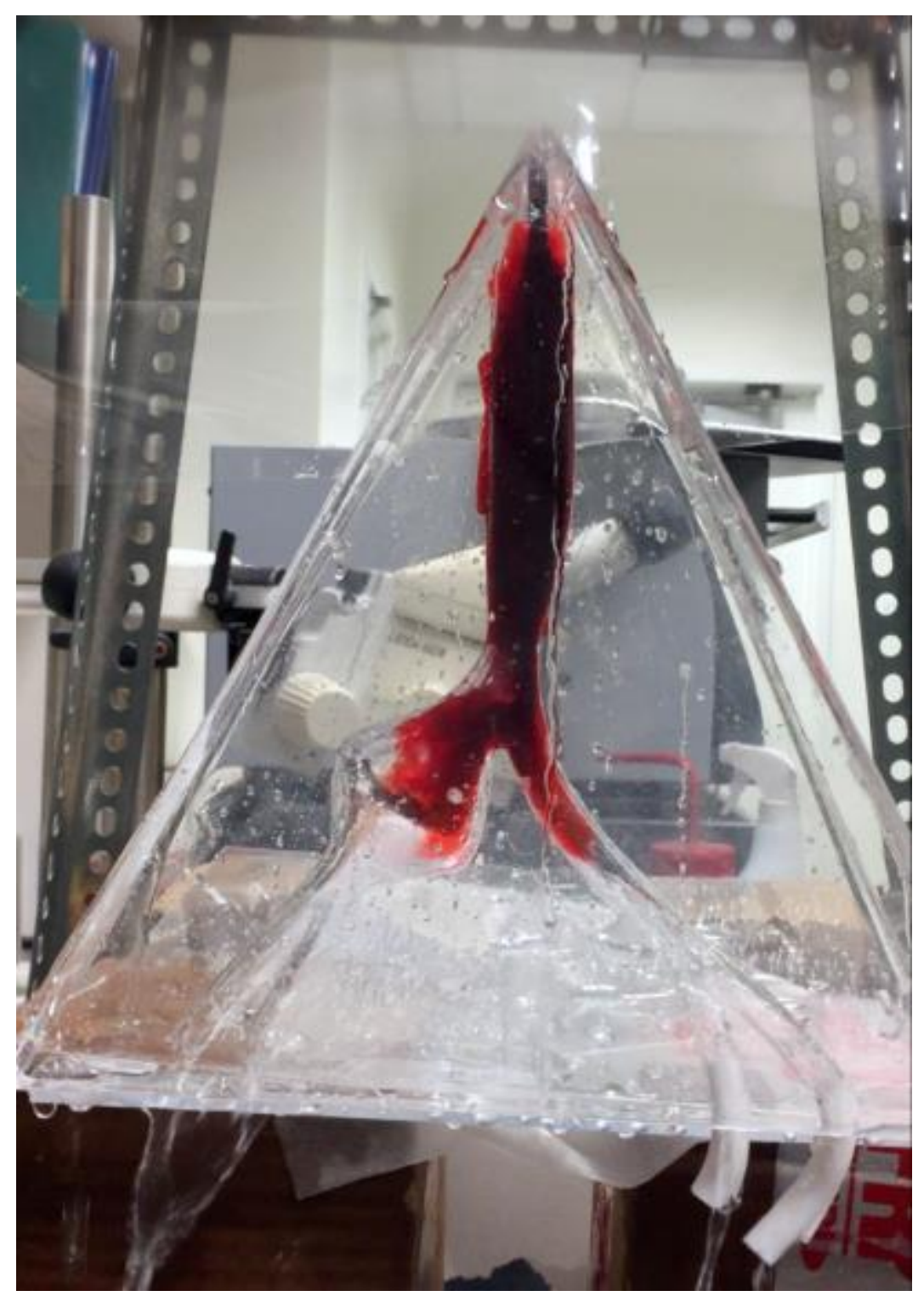




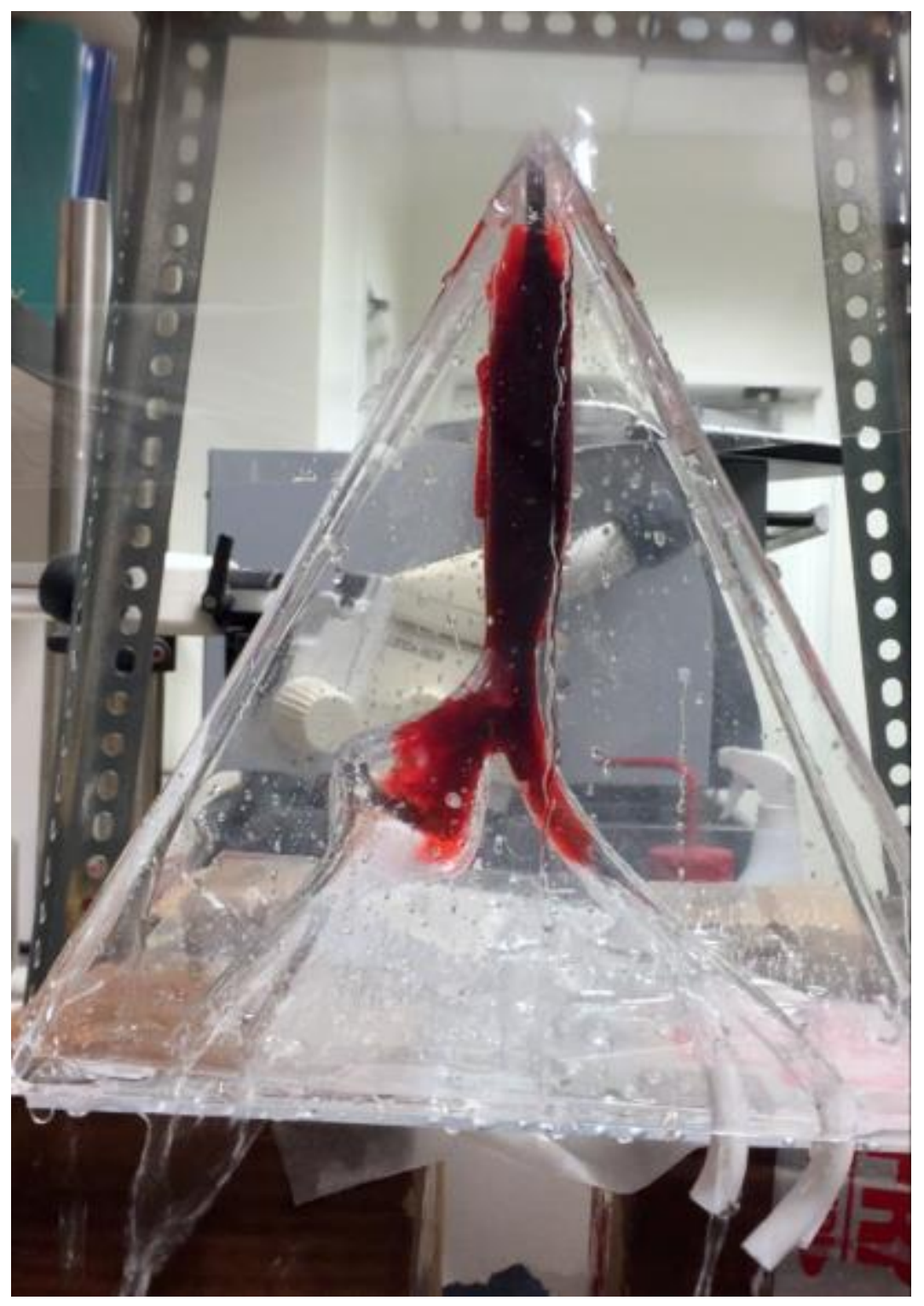




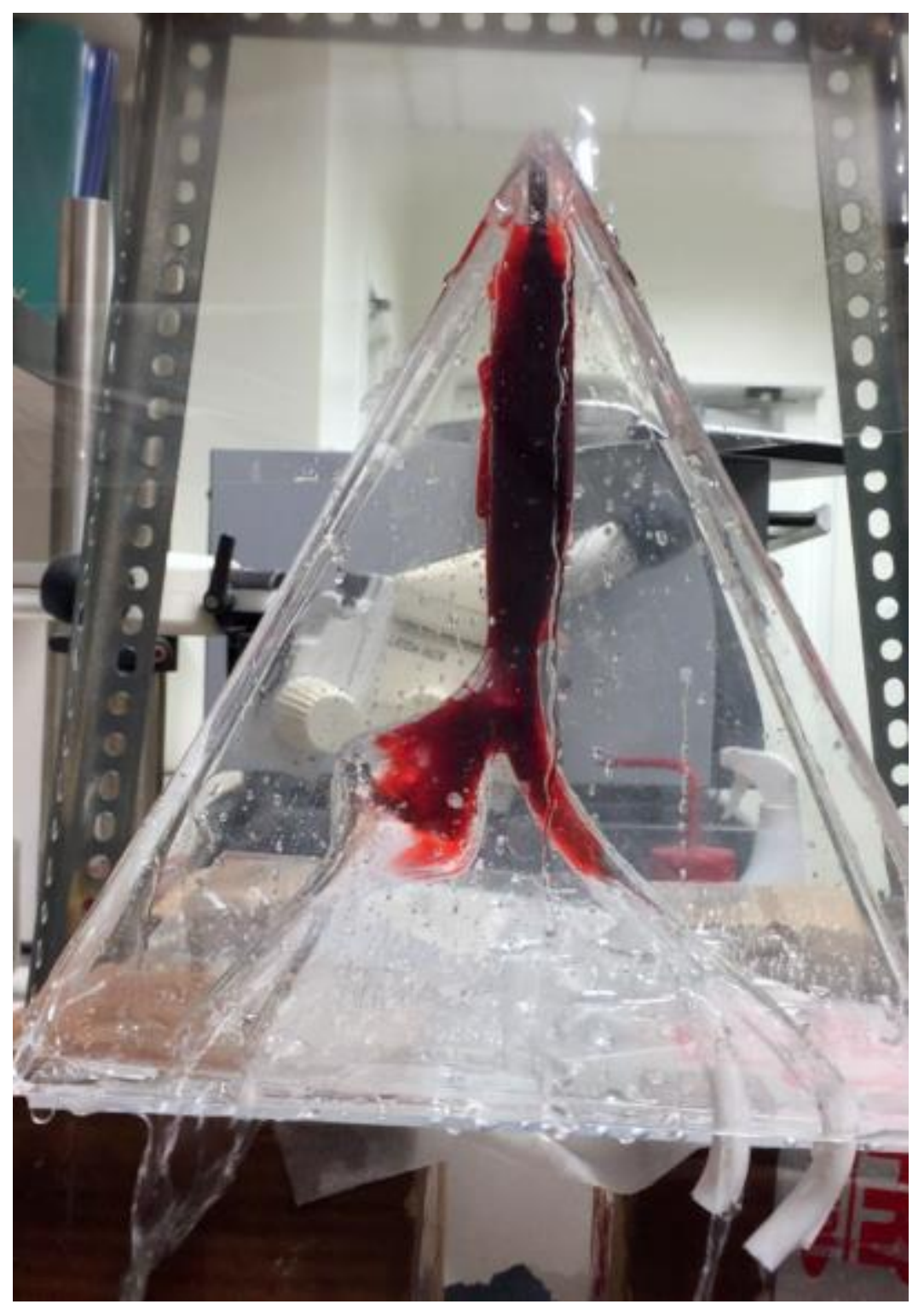




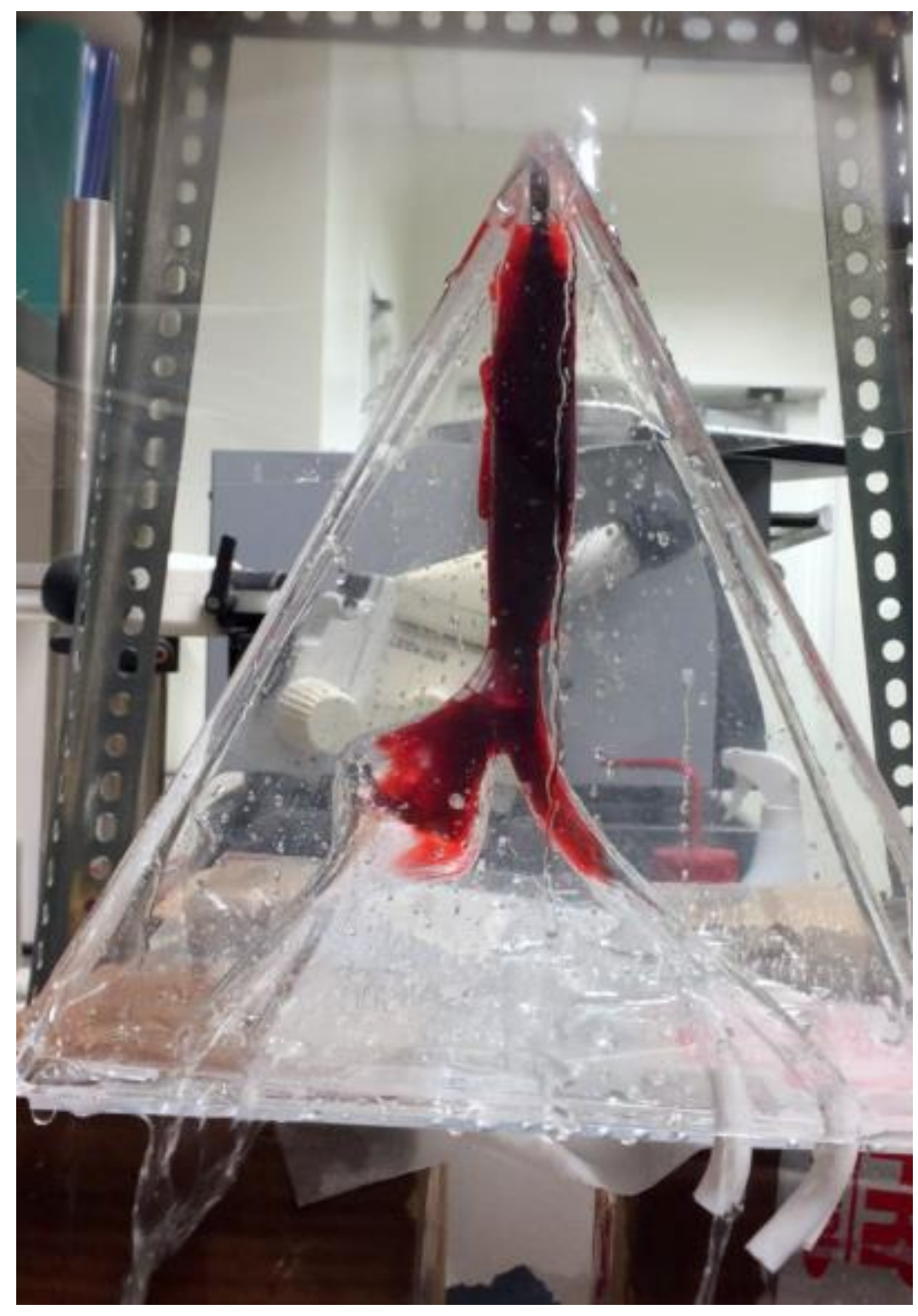




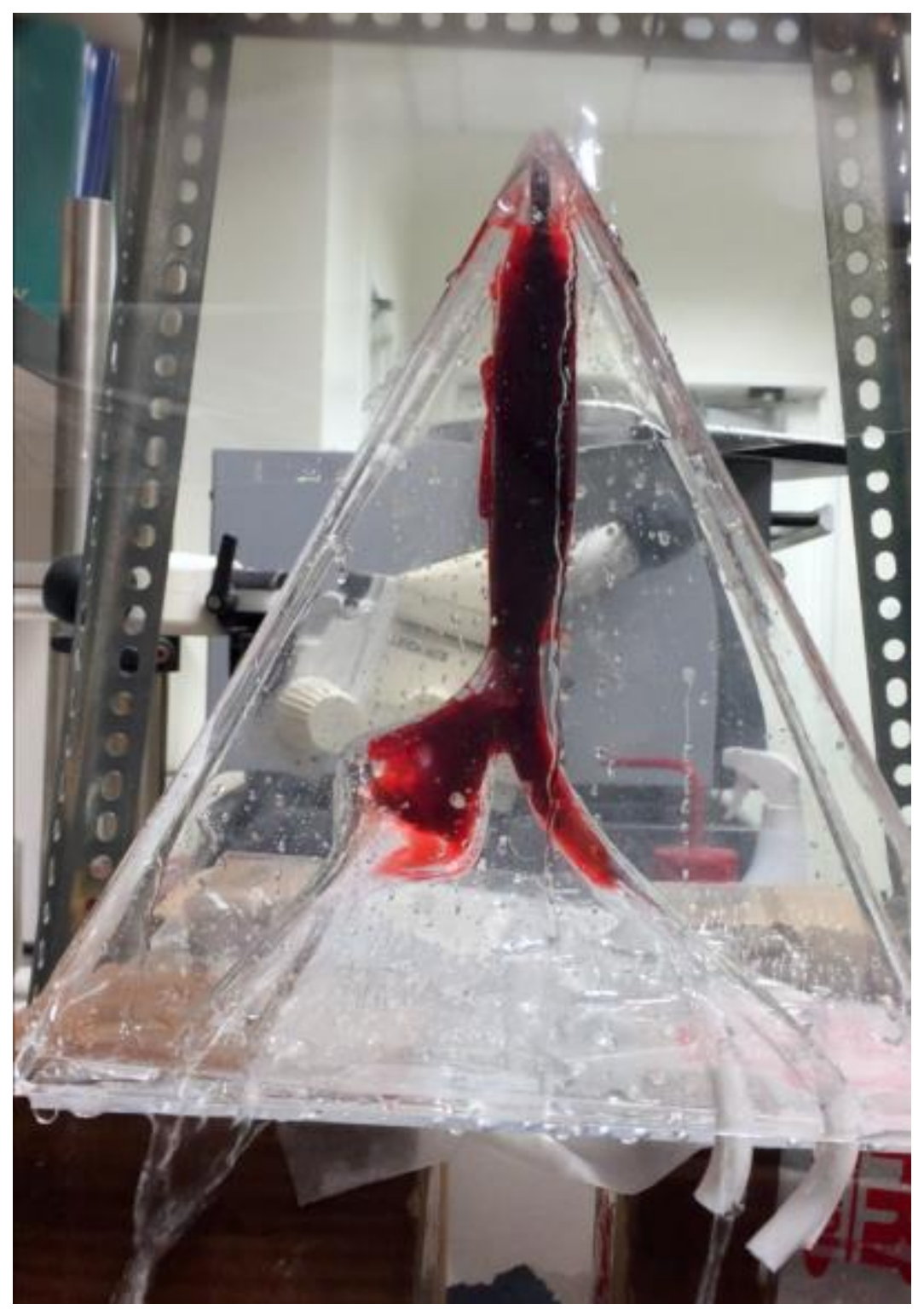




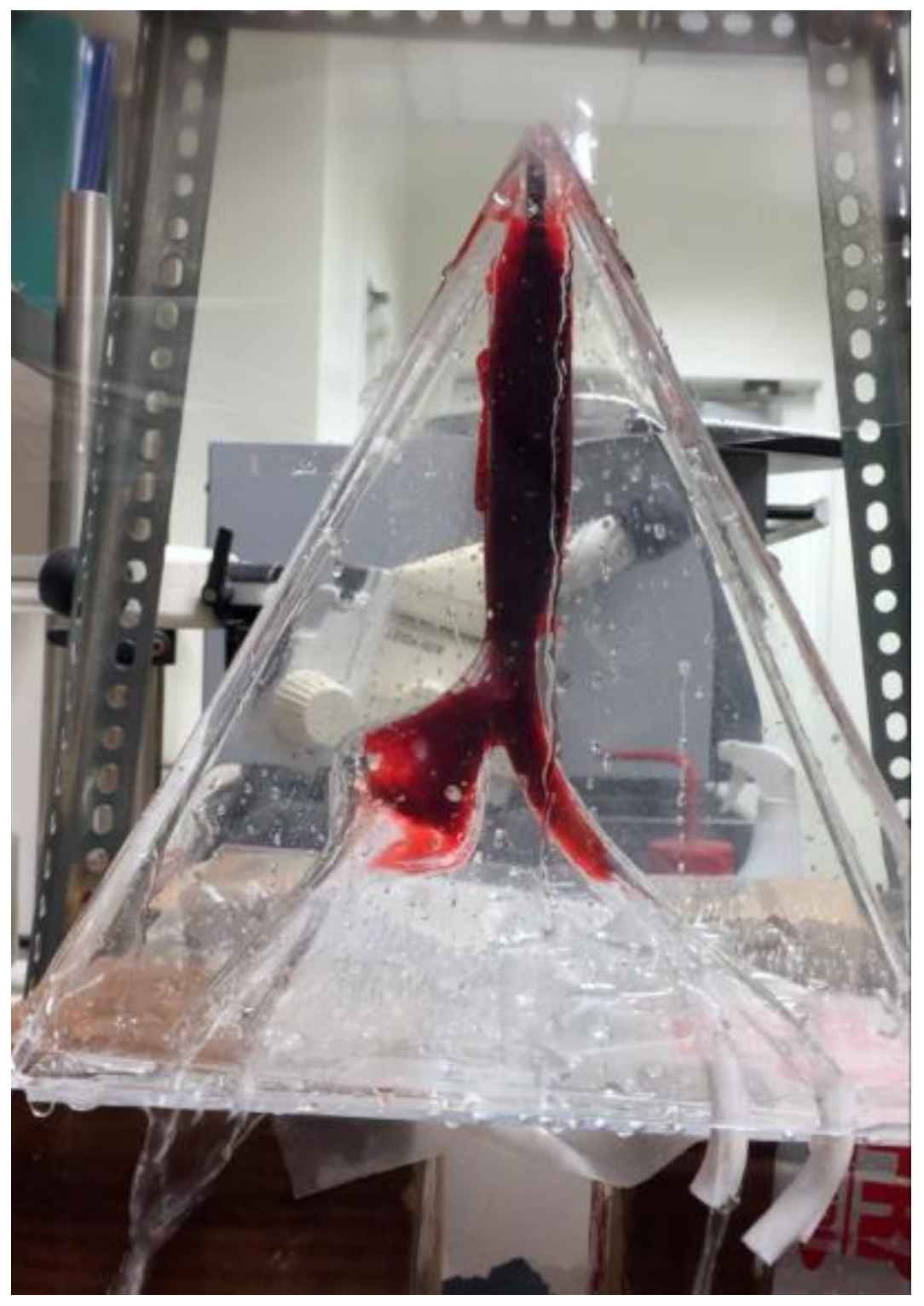




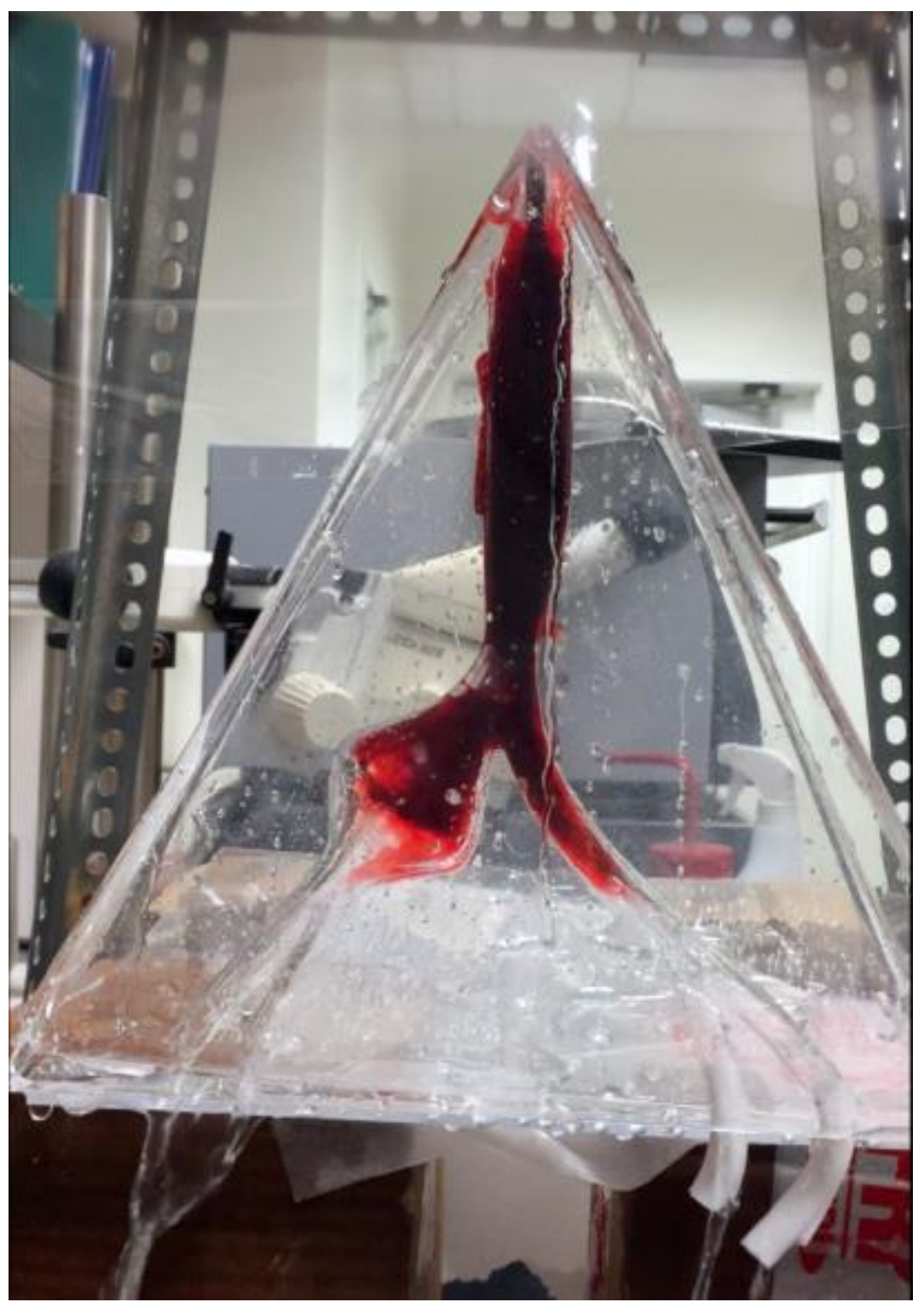




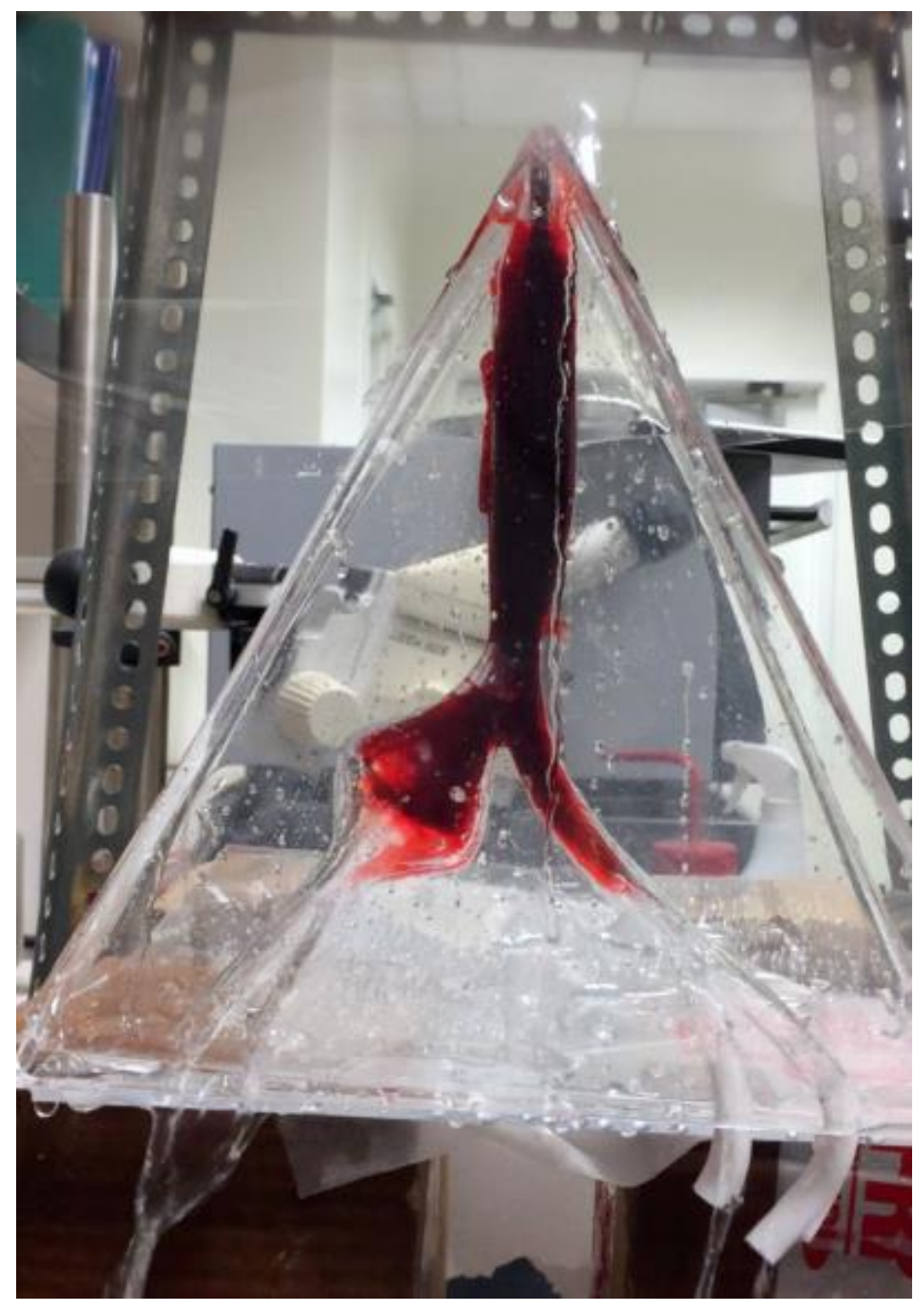




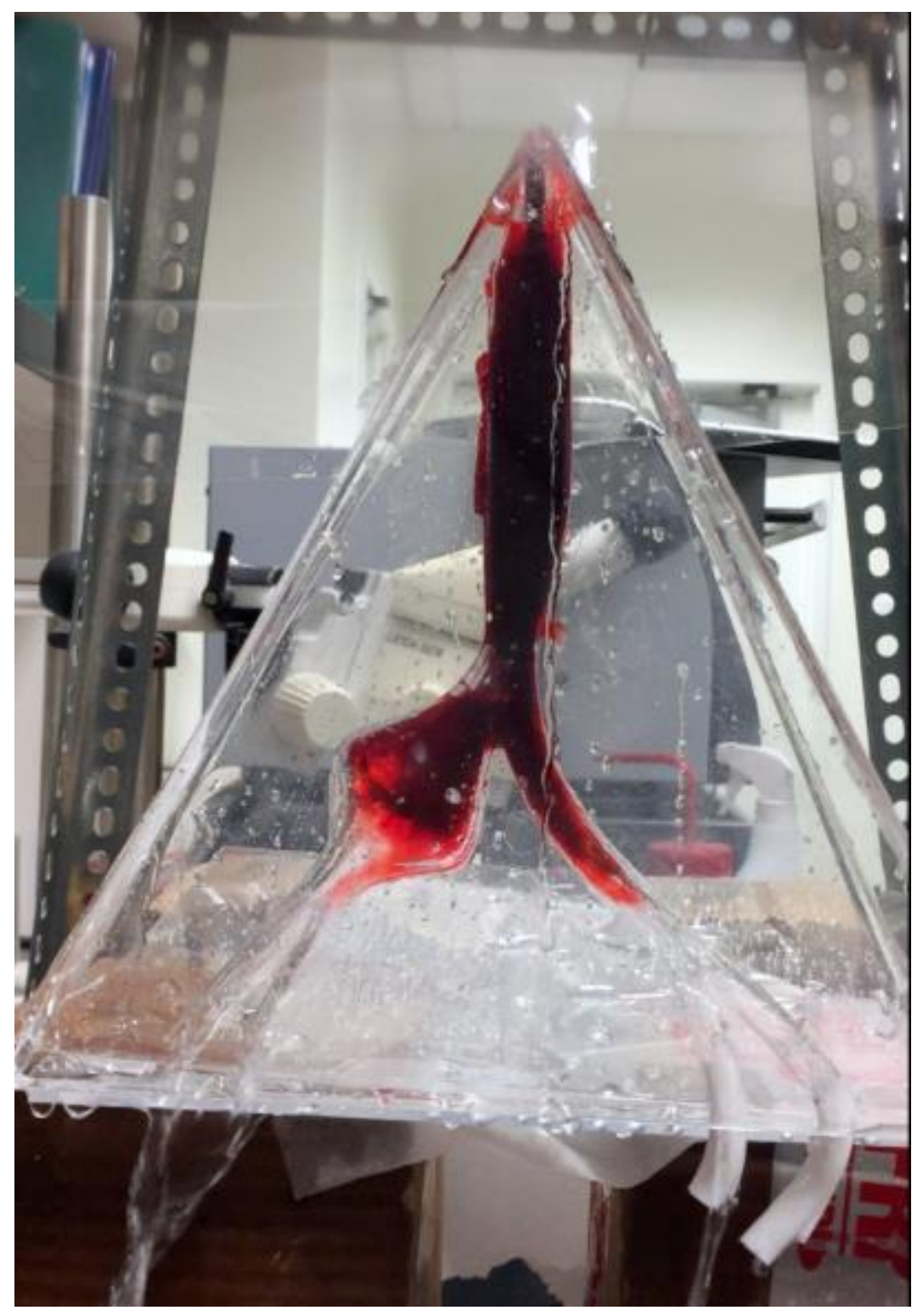




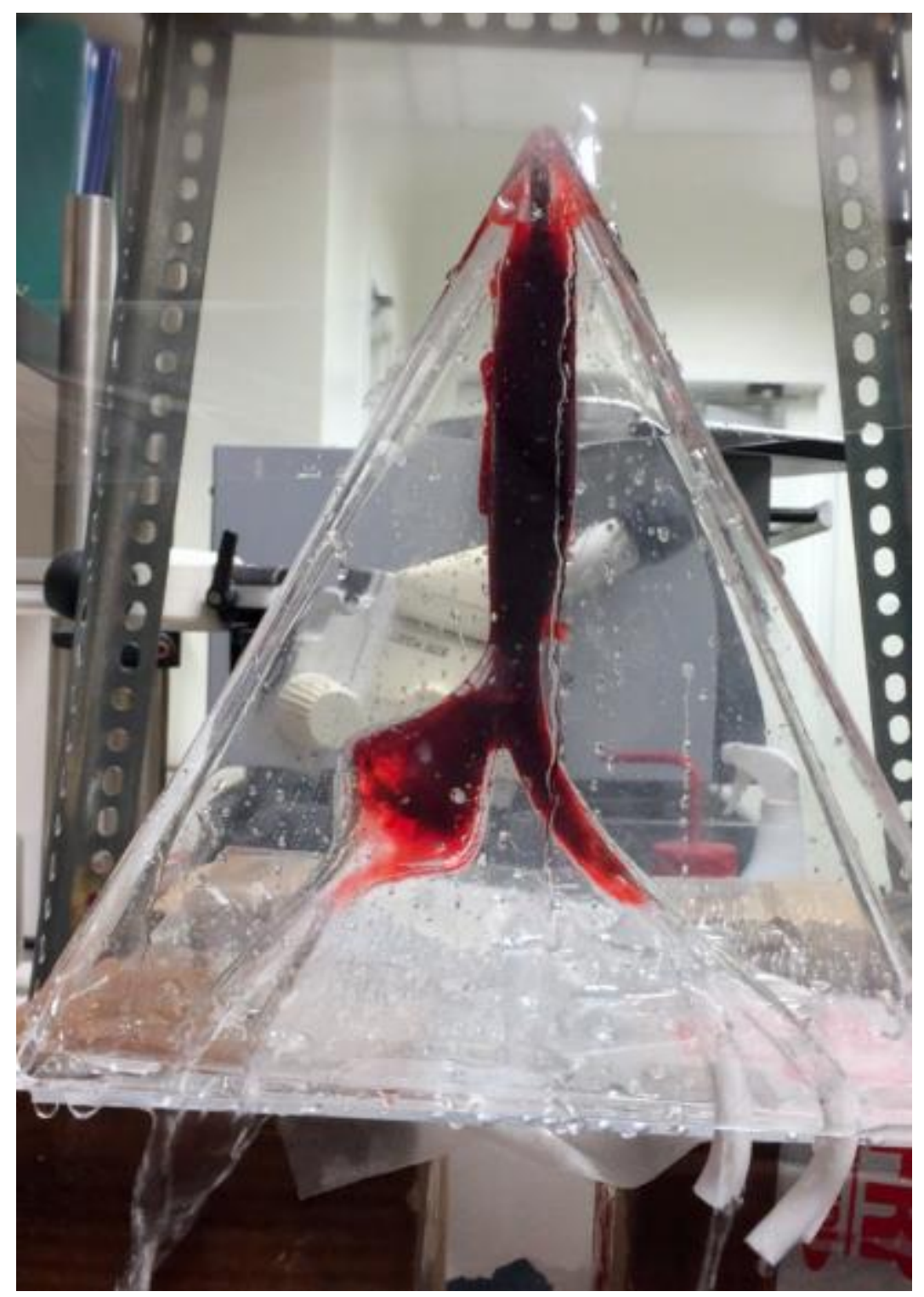




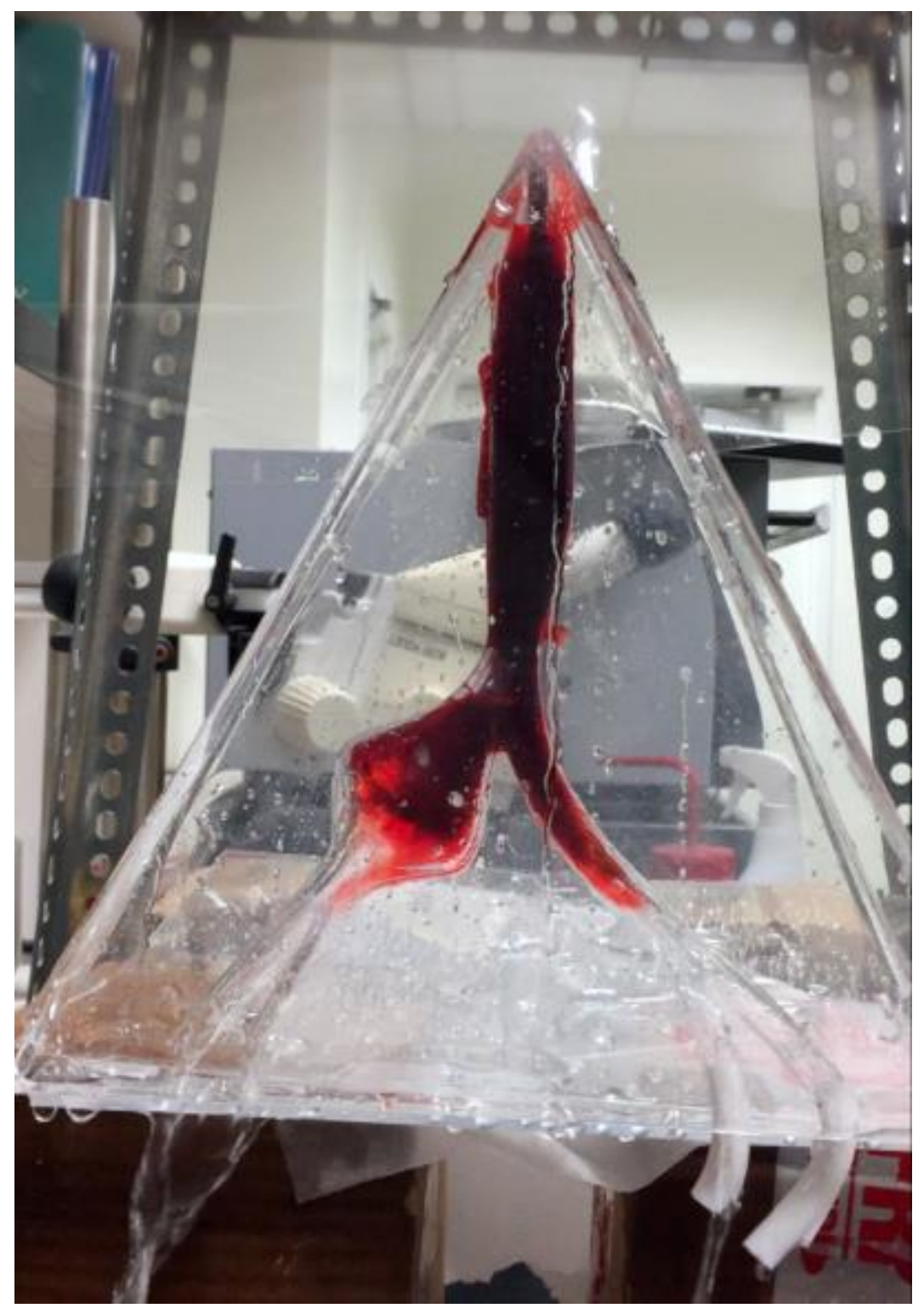




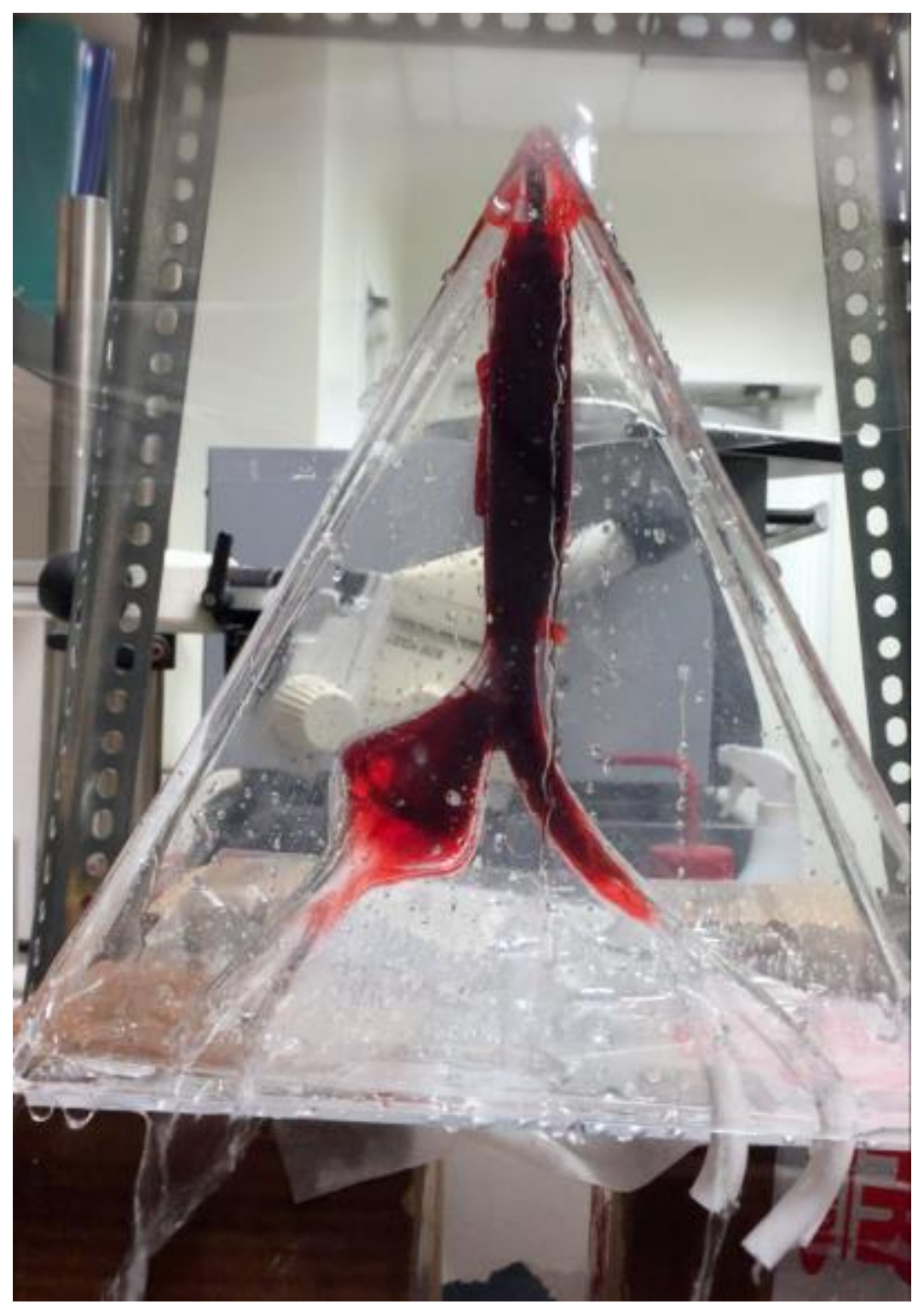




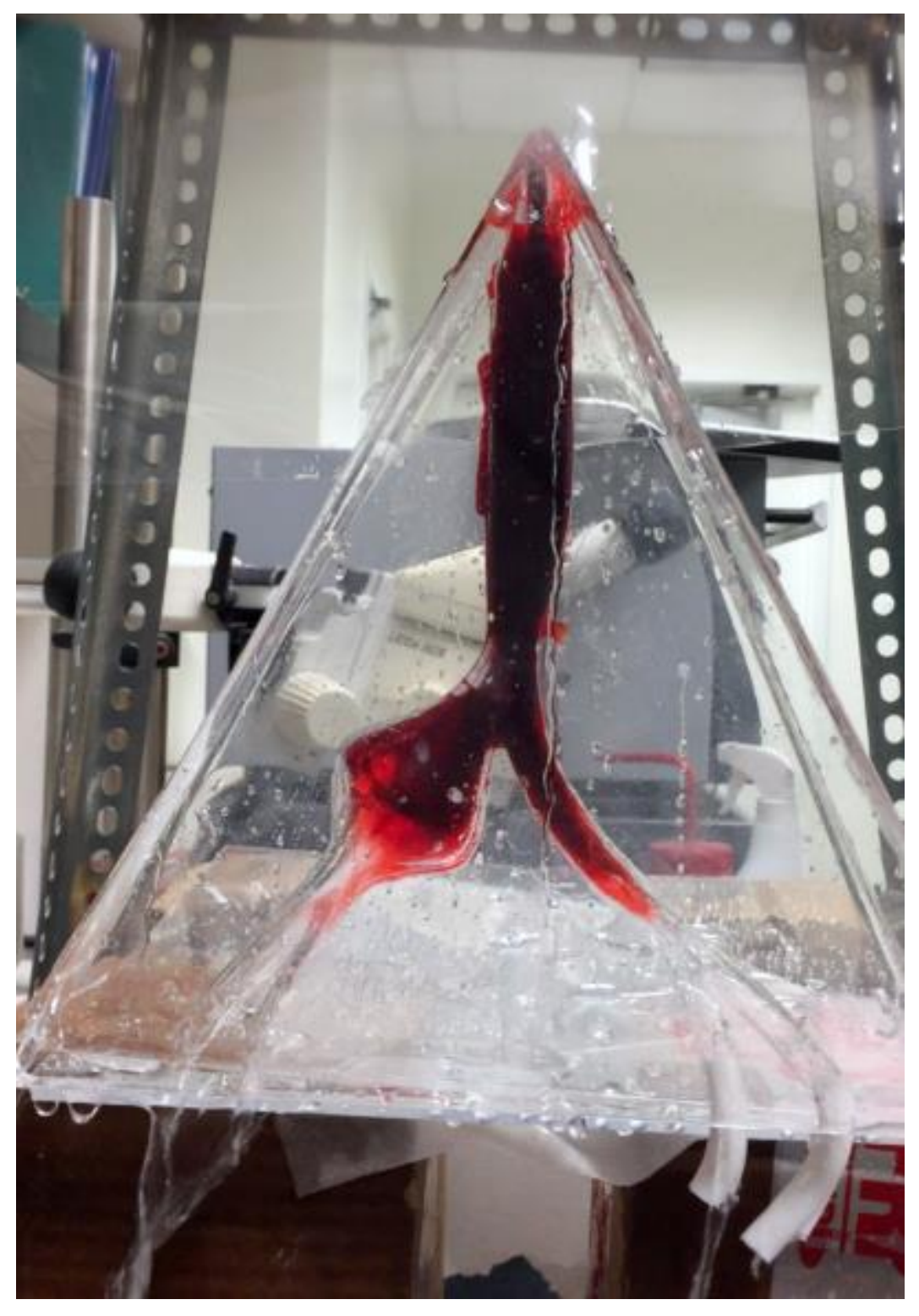




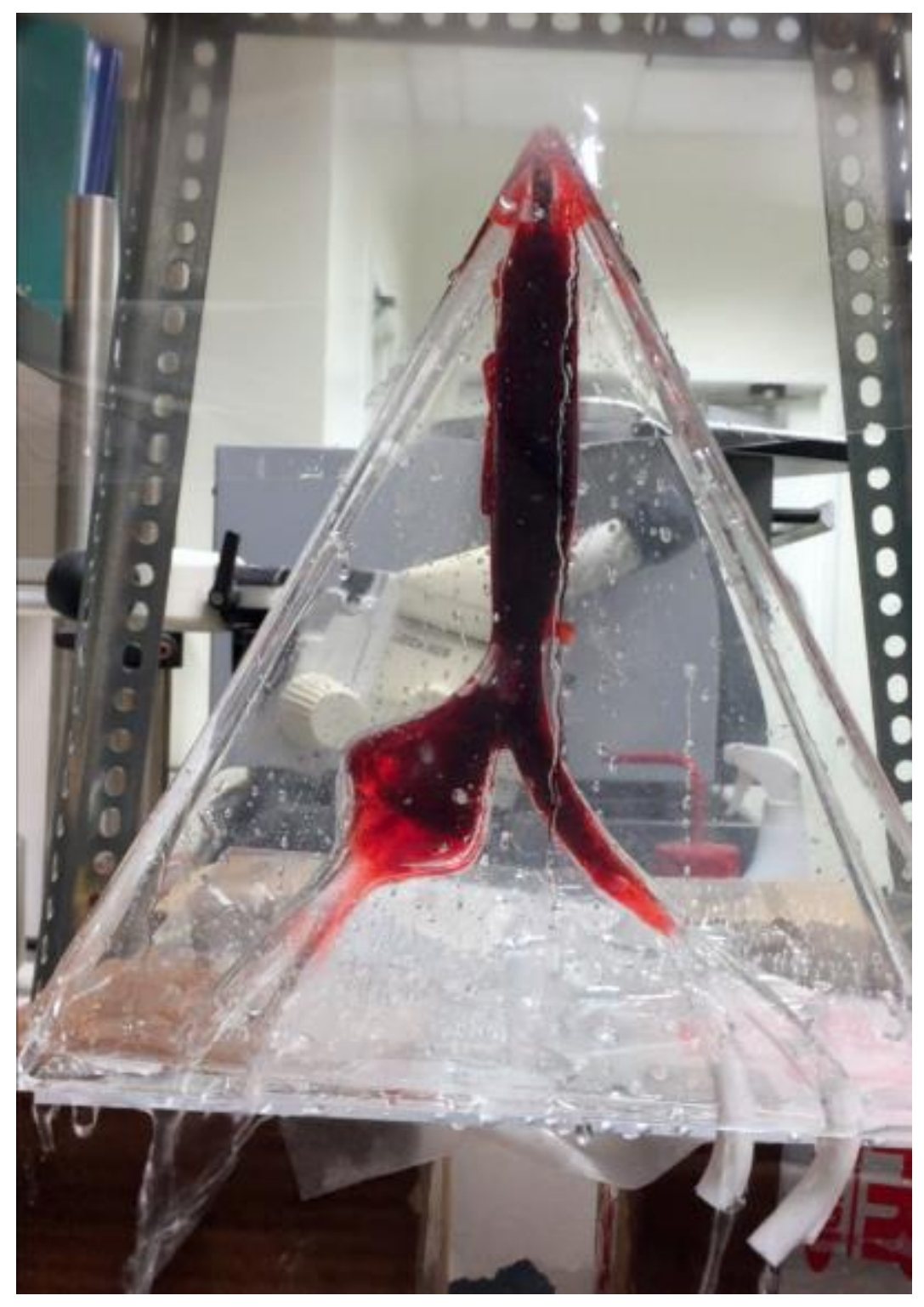




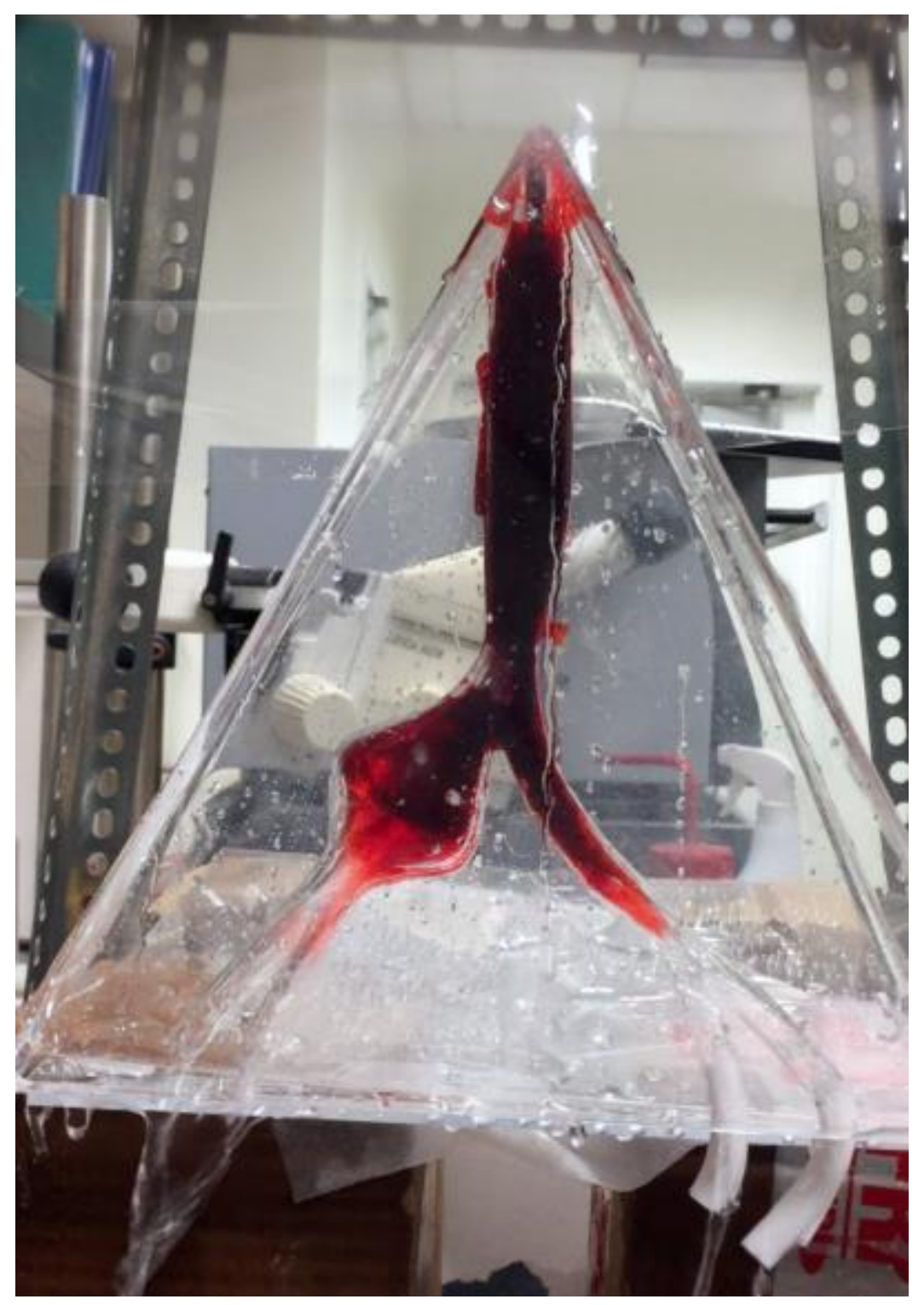


Two-sample T for Shear Stress on the outer edge of Aneurysmal (C1) vs NonAneurysmal (C2)

$\mathrm{N}$ Mean StDev SE Mean

C1 $6011.2450 .695 \quad 0.028$

C2 $601 \quad 1.2450 .695 \quad 0.028$

Difference $=\mathrm{mu}(\mathrm{C} 1)-\mathrm{mu}(\mathrm{C} 2)$

Estimate for difference: 0.0000

$95 \% \mathrm{Cl}$ for difference: $(-0.0787,0.0787)$

$\mathrm{T}$-Test of difference $=0($ vs not $=): \mathrm{T}$-Value $=0.00 \mathrm{P}$-Value $=1.000 \mathrm{DF}=1200$

Two-sample T for Shear Stress on the inner edge of Aneurysmal (C1) vs NonAneurysmal (C2)

$\mathrm{N}$ Mean StDev SE Mean

C1 $181 \quad 1.891 .81 \quad 0.13$

C2 $1812.217 \quad 0.361 \quad 0.027$

Difference $=\mathrm{mu}(\mathrm{C} 1)-\mathrm{mu}(\mathrm{C} 2)$

Estimate for difference: -0.323

$95 \% \mathrm{Cl}$ for difference: $(-0.594,-0.052)$

T-Test of difference $=0($ vs not $=): T$-Value $=-2.35 P$-Value $=0.020 \mathrm{DF}=194$

Two-sample T for COMSOL MULTIPHYSICS $®$ Velocity (C1) vs Measured Velocity (C2)

$\mathrm{N}$ Mean StDev SE Mean

$\begin{array}{lllll}C 1 & 19 & 0.205 & 0.161 & 0.037\end{array}$

C2 $190.2510 .177 \quad 0.041$

Difference $=\mathrm{mu}(\mathrm{C} 1)-\mathrm{mu}(\mathrm{C} 2)$

Estimate for difference: -0.0465

$95 \% \mathrm{Cl}$ for difference: $(-0.1582,0.0653)$

$\mathrm{T}$-Test of difference $=0($ vs not $=): \mathrm{T}-$ Value $=-0.84 \mathrm{P}$-Value $=0.404 \mathrm{DF}=35$

Paired Two-sample T for Shear Stress on the outer edge of Aneurysmal (C1) vs NonAneurysmal (C2)

N Mean StDev SE Mean 
$\begin{array}{lllll}\text { C1 } & 601 & 1.2451 & 0.6952 & 0.0284\end{array}$

$\begin{array}{lllll}\mathrm{C} 2 & 601 & 1.2451 & 0.6952 & 0.0284\end{array}$

Difference $6010.000000 \quad 0.0000000 .000000$

$95 \% \mathrm{Cl}$ for mean difference: $(0.000000,0.000000)$

$\mathrm{T}$ - Test of mean difference $=0($ vs not $=0): \mathrm{T}$-Value $=4.68 \mathrm{P}$-Value $=0.000$

Paired Two-sample T for Shear Stress on the inner edge of Aneurysmal (C1) vs NonAneurysmal (C2)

Paired T for $\mathrm{C} 1$ - C2

$\mathrm{N}$ Mean StDev SE Mean

C1 $\quad \begin{array}{lllll}181 & 1.894 & 1.811 & 0.135\end{array}$

$\begin{array}{lllll}\text { C2 } & 181 & 2.217 & 0.361 & 0.027\end{array}$

Difference $181-0.323 \quad 1.598 \quad 0.119$

$95 \% \mathrm{Cl}$ for mean difference: $(-0.557,-0.089)$

$\mathrm{T}$-Test of mean difference $=0($ vs not $=0): \mathrm{T}-$ Value $=-2.72 \mathrm{P}$-Value $=0.007$

Paired Two-sample T for COMSOL MULTIPHYSICS $®$ Velocity (C1) vs Measured Velocity (C2)

Paired T for C1 - C2

Paired T for C1 - C2

$N$ Mean StDev SE Mean

C1 $\quad 19 \quad 0.1966 \quad 0.1559 \quad 0.0358$

$\begin{array}{lllll}\text { C2 } & 19 & 0.2513 & 0.1775 & 0.0407\end{array}$

Difference $19-0.0546 \quad 0.2472 \quad 0.0567$

$95 \% \mathrm{Cl}$ for mean difference: $(-0.1738,0.0645)$

T-Test of mean difference $=0($ vs not $=0): T-$ Value $=-0.96 \mathrm{P}$-Value $=0.348$ 


\section{APPENDIX D: Calculations}

Entrance Length Calculation

$$
\begin{gathered}
\frac{L_{e}}{d}=4.4 R e^{1 / 6} \\
\frac{L_{e}}{0.5 \mathrm{~cm}}=4.4(1905)^{1 / 6}
\end{gathered}
$$

Inlet Velocity Calculation

$$
\frac{(0.5 * 0.02 \mathrm{~m})^{2} * \pi}{2} * \frac{2.258 \mathrm{~L}}{\min } * \frac{1 \mathrm{~m}^{3}}{1000 \mathrm{~L}} * \frac{60 \mathrm{sec}}{\min }=0.2397 \mathrm{~m} / \mathrm{s}
$$

Reynolds Number Calculation

$$
R e=\frac{\rho v D}{\mu}=\frac{\frac{1000 \mathrm{~kg}}{\mathrm{~m}^{3}} * \frac{0.2397 \mathrm{~m}}{\mathrm{~s}} * 0.0079}{1.0 \times 10^{-3} \mathrm{~Pa} * S}=1905
$$


APPENDIX E: ImageJ Thresholding Processes Described in Thumbnails
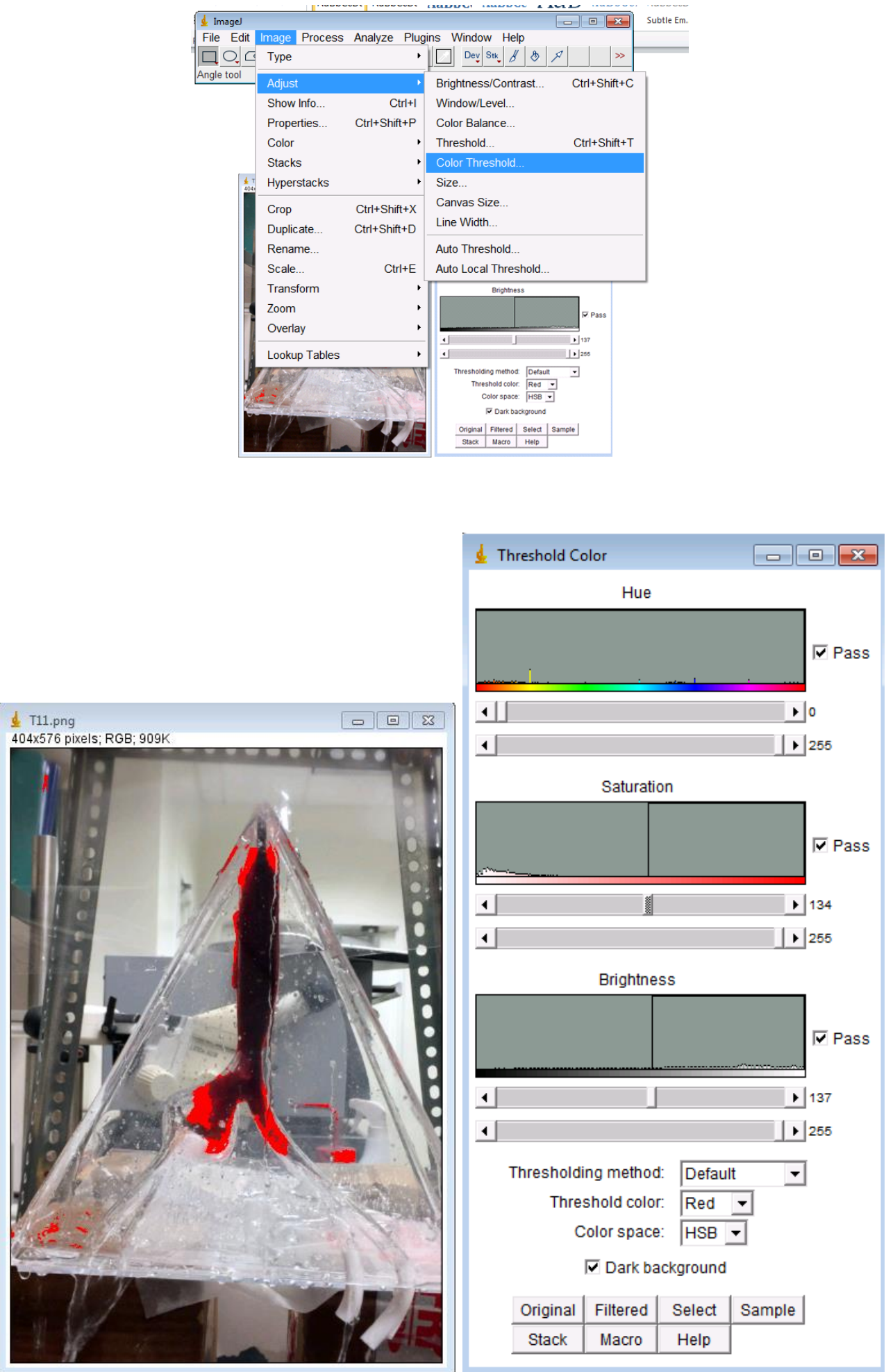